\title{
Measurement of $\pi-K$ Ratios from the NuMI Target
}

\author{
A dissertation presented \\ by
}

Sin Man Seun

to

The Department of Physics

in partial fulfillment of the requirements

for the degree of

Doctor of Philosophy

in the subject of

Physics

Harvard University

Cambridge, Massachusetts

July 2007 
(C)2007 - Sin Man Seun

All rights reserved. 
Dissertation Advisor

Author

Professor Gary J. Feldman

Sin Man Seun

\title{
Measurement of $\pi-K$ Ratios from the NuMI Target
}

\begin{abstract}
Interactions of protons (p) with the NuMI (Neutrinos at the Main Injector) [1] target are used to create the neutrino beam for the MINOS (Main Injector Neutrino Oscillation Search) [2] Experiment. Using the MIPP (Main Injector Particle Production) experimental apparatus, the production of charged pions and kaons in $\mathrm{p}+\mathrm{NuMI}$ interactions is studied. The data come from a sample of $2 \times 10^{6}$ events obtained by MIPP using the $120 \mathrm{GeV} / c$ proton beam from the Main Injector at Fermi National Accelerator Laboratory in Illinois, USA. Pions and kaons are identified by measurement in a Ring Imaging Cherenkov detector.

Presented are measurements of $\pi^{-} / \pi^{+}, K^{-} / K^{+}, \pi^{+} / K^{+}$and $\pi^{-} / K^{-}$production ratios in the momentum range $p_{T}<2 \mathrm{GeV} / c$ transversely and $20 \mathrm{GeV} / c<$ $p_{z}<90 \mathrm{GeV} / c$ longitudinally. Also provided are detailed comparisons of the MIPP NuMI data with the MIPP Thin Carbon data [3], the MIPP Monte Carlo simulation and the current MINOS models $[4,5]$ in the relevant momentum ranges.
\end{abstract}




\section{Contents}

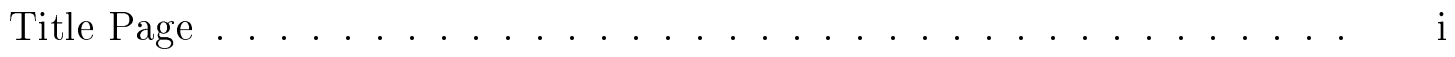

Abstract .......................... iii

Table of Contents . . . . . . . . . . . . . . . iv

List of Figures . . . . . . . . . . . . . . . . . vi

List of Tables . . . . . . . . . . . . . . . . ix

Acknowledgments . . . . . . . . . . . . . . . .

1 Introduction 1

2 Particle Production 3

2.1 Particle Production Data from Related Experiments . . . . . . . . . 3

2.2 Simulations of Particle Production from the NuMI Target and Uncertainties in the NuMI Neutrino Spectra . . . . . . . . . 5

3 The MIPP Experiment $\quad 8$

3.1 The Beamline . . . . . . . . . . . . . . . . . . . . 9

3.1 .1 Primary Beamline . . . . . . . . . . . . . . . . . 10

3.1.2 Secondary Beamline . . . . . . . . . . . . . . . . . 11

3.1 .3 Beam Cherenkov . . . . . . . . . . . . . . . . . . . . 12

3.2 Targets . . . . . . . . . . . . . . . . . . . . . . 13

3.3 The Detectors . . . . . . . . . . . . . . . . . . . . 16

3.3 .1 Time Projection Chamber . . . . . . . . . . . . . 17

3.3 .2 Time-Of-Flight . . . . . . . . . . . . . . . . . . . . 21

3.3 .3 Cherenkov . . . . . . . . . . . . . . . . . . . . . 21

3.3.4 Ring Imaging Cherenkov Detector . . . . . . . . . . . . . . . 23

3.3.5 Electromagnetic and Hadronic Calorimeter . . . . . . . . . . 29

3.3.6 Drift and Proportional Chambers . . . . . . . . . . . . 32

3.3 .7 Magnets . . . . . . . . . . . . . . . . . . . 33

3.4 MIPP Acceptances for NuMI Target . . . . . . . . . . . . . 34 
4 Event Reconstruction $\quad 36$

4.1 Monte Carlo Simulation . . . . . . . . . . . . . . . . 37

4.2 Tracking . . . . . . . . . . . . . . . . . . 37

4.3 Particle Identification . . . . . . . . . . . . . . . . . . . . . . . . . . 39

4.3.1 The Cherenkov Effect. . . . . . . . . . . . . . 41

4.3.2 Detector Effect . . . . . . . . . . . . . . . . 43

4.3.3 Maximum Likelihood Method for Particle Identification . . . . 47

4.3.4 Iterative Weighting Method . . . . . . . . . . . . . . 50

4.4 RICH Alignment . . . . . . . . . . . . . . . . . . . 52

4.5 RICH Calibration . . . . . . . . . . . . . . . . 53

$5 \quad$ NuMI Target Analysis $\quad 57$

5.1 Event Sample and Cuts . . . . . . . . . . . . . . 57

5.1 NuMI Trigger Scheme and Beam Selection . . . . . . . . 58

5.1 .2 Track Selection . . . . . . . . . . . . . 58

5.1.3 Momentum Selection and Binning . . . . . . . . . . 61

5.1.4 Particle Classification . . . . . . . . . . . . . . . 62

5.2 Evaluation of Corrections . . . . . . . . . . . . . . 64

5.2.1 Purity Correction ................... 69

5.2 .2 Momentum Correction . . . . . . . . . . . . . . 71

5.2.3 Efficiency Correction . . . . . . . . . . . . . . . 73

5.2.4 Application of Corrections . . . . . . . . . . . . . 73

5.2.5 Background Estimation from Data . . . . . . . . . . . 73

5.3 Systematic Errors . . . . . . . . . . . . . . . . . . 81

5.3.1 Beam Systematic Error . . . . . . . . . . . . . 81

5.3.2 Momentum Systematic Error . . . . . . . . . . . . . 84

5.3.3 Background Systematic Error . . . . . . . . . . . . 86

6 Results and Comparison $\quad 90$

6.1 Measurements of Particle Production Ratios from the NuMI Target . 90

6.2 Comparison to Other Data and Models . . . . . . . . . . . . 96

$\begin{array}{lll}7 & \text { Conclusions } & 103\end{array}$

$\begin{array}{ll}\text { A Maximization of Purity and Efficiency } & 105\end{array}$

$\begin{array}{ll}\text { Bibliography } & 107\end{array}$ 


\section{List of Figures}

2.1 The distribution in $p$ and $p_{T}$ for secondary pions produced on the NuMI

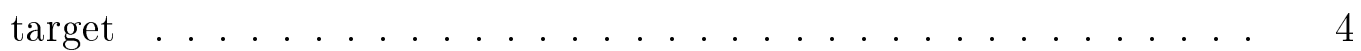

2.2 Predictions of the absolute neutrino rates at the MINOS Near detector 6

2.3 Predictions of the ratio of the Far neutrino flux to the Near neutrino flux using four hadron production models . . . . . . . . . . . 7

3.1 The elements and an elevation view of the MIPP beamline . . . . . 9

3.2 Schematic of the NuMI target design . . . . . . . . . . . . . . 15

3.3 Layout of the MIPP experiment . . . . . . . . . . . . . . . 17

3.4 Plan view of the E907 detectors . . . . . . . . . . . . . . . . . . 18

3.5 Exploded view of the TPC with the outer case removed . . . . . . . . 19

3.6 Sketch of the wire planes and field lines . . . . . . . . . . . . . 20

3.7 The Cherenkov detector . . . . . . . . . . . . . . . . . . 22

3.8 A schematic view of the RICH detector . . . . . . . . . . . . . . . 24

3.9 Online display in the RICH detector . . . . . . . . . . . . . . . . 24

3.10 Mirror layout in the vessel . . . . . . . . . . . . . . . . . 26

3.11 The phototube holder plate . . . . . . . . . . . . . . . 27

3.12 Side view sketch of the calorimeters . . . . . . . . . . . . . . 30

3.13 Hadronic Calorimeter details . . . . . . . . . . . . . . . . . . . . . 32

3.14 The acceptance of the various sub-detectors in the MIPP experiment as a function of secondary particle momentum and transverse momentum 35

$3.15 p_{t}$ vs. $p$ spectrum for tracks coming off the NuMI target . . . . . . 35

4.1 Effect of JGG magnetic field on particle production . . . . . . . . . 38

4.2 Event display in the RICH . . . . . . . . . . . . . . . . . . . . . 40

4.3 Index of refraction of $\mathrm{CO}_{2}$ as a function of wavelength . . . . . . . . 42

4.4 The reflectance at normal incidence from mirrors . . . . . . . . . . 44

4.5 Absorption region of Carbon Dioxide . . . . . . . . . . . . . . . . . . 44

4.6 Efficiencies of light transmission of a PMT and its housing as a function of wavelength . . . . . . . . . . . . . . . . . . . 45 
4.7 RICH alignment constants versus mirror numbers . . . . . . . . . 53

4.8 The RICH calibration for Russian PMTs . . . . . . . . . . . . . 55

4.9 The RICH calibration for Hamamatsu PMTs . . . . . . . . . . . . . 56

5.1 A schematic of the NuMI triggers . . . . . . . . . . . . . 59

5.2 Beam positions of $2 \mathrm{~mm}$ and $6 \mathrm{~mm}$ NuMI triggers . . . . . . . . 59

5.3 Radial and longitudinal reconstructed vertex positions for interaction vertices with $\geq 2$ tracks in data . . . . . . . . . . . . . 60

$5.4 \log$ likelihood ratios for $\pi^{+}, \pi^{-}, K^{+}$and $K^{-}$with respect to $\mathrm{p}$ in Monte Carlo . . . . . . . . . . . . . . . . . 63

5.5 Log-likelihood ratio for reconstructed $\pi^{+}$in different momentum bins $\quad 65$

5.6 Log-likelihood ratio for reconstructed $\pi^{-}$in different momentum bins $\quad 66$

5.7 Log-likelihood ratio for reconstructed $K^{+}$in different momentum bins $\quad 67$

5.8 Log-likelihood ratio for reconstructed $K^{-}$in different momentum bins $\quad 68$

5.9 Purity of $\pi^{ \pm}, K^{ \pm}$and $p^{ \pm}$as a function of reconstructed momentum

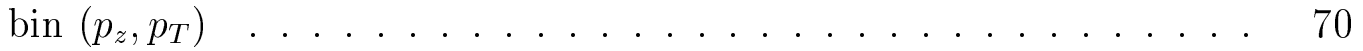

5.10 Momentum correction factors of $\pi^{ \pm}, K^{ \pm}$and $p^{ \pm}$as a function of true momentum bin versus reconstructed momentum bin . . . . . . . . . . 72

5.11 Efficiency of $\pi^{ \pm}, K^{ \pm}$and $p^{ \pm}$selection as a function of true momentum

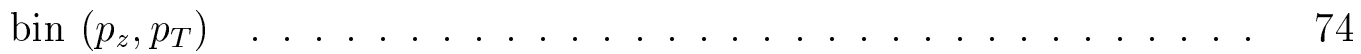

5.12 Yield for each particle species in data $\left(f_{\pi^{ \pm}}, f_{K^{ \pm}}, f_{p^{ \pm}}\right)$and in Monte Carlo $\left(F_{\pi^{ \pm}}, F_{K^{ \pm}}\right.$, and $\left.F_{p^{ \pm}}\right) \ldots \ldots \ldots$. . . . . . . . 76

5.13 Effect of background subtraction on fractional change in yield ratio . 78

5.14 Effect of Monte Carlo event weighting on purity correction factor . . 79

5.15 Normalized number of entries for $\pi^{ \pm}, K^{ \pm}$and $p^{ \pm}$as a function of momentum bin ................. . . 80

5.16 Reconstructed beam positions and their Gaussian fits in data and Monte Carlo simulation . . . . . . . . . . . . . . . . . . . . 82

5.17 Effect of beam systematic on $\pi^{+} / \pi^{-}, K^{+} / K^{-}, K^{+} / \pi^{+}$and $K^{-} / \pi^{-}$.

5.18 Reconstructed momentum and its corresponding Gaussian fit in data and Monte Carlo simulation for $120 \mathrm{GeV} / c$ proton beam . . . . . . . 84

5.19 Effect of momentum systematic on $\pi^{+} / \pi^{-}, K^{+} / K^{-}, K^{+} / \pi^{+}$and $K^{-} / \pi^{-} 85$

5.20 Effect of background systematic on $\pi^{+} / \pi^{-}, K^{+} / K^{-}, K^{+} / \pi^{+}$and $K^{-} / \pi^{-}$ due to change in purity . . . . . . . . . . . . . . . . .

5.21 Effect of background systematic on $\pi^{+} / \pi^{-}, K^{+} / K^{-}, K^{+} / \pi^{+}$and $K^{-} / \pi^{-}$ due to change in log-likelihood ratio cuts . . . . . . . . . .

6.1 The ratios as a function of $\left(p_{z}, p_{T}\right)$ for $\pi^{-} / \pi^{+}, K^{-} / K^{+}$and $K^{ \pm} / \pi^{ \pm}$ produced in $\mathrm{p}+\mathrm{NuMI}$ collisions at $120 \mathrm{GeV} / c \ldots . . . . . .$.

6.2 The ratios as a function of $\left(p_{z}, p_{T}\right)$ for $\pi^{-} / \pi^{+}, K^{-} / K^{+}$and $K^{ \pm} / \pi^{ \pm}$ produced in $\mathrm{p}+\mathrm{NuMI}, \mathrm{p}+\mathrm{C}$ collisions and NuMI Monte Carlo simulation at $120 \mathrm{GeV} / c \ldots \ldots \ldots \ldots$ 
6.3 The ratios between $\mathrm{p}+\mathrm{NuMI}$ and $\mathrm{p}+\mathrm{C}$ collisions as a function of $\left(p_{z}, p_{T}\right)$ for $\pi^{-} / \pi^{+}, K^{-} / K^{+}$and $K^{ \pm} / \pi^{ \pm}$in data and Monte Carlo simulation

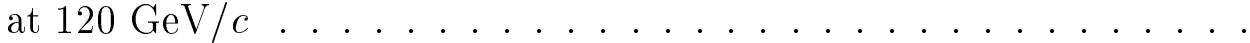

6.4 The ratios as a function of $p_{z}$ at $p_{T}<0.2 \mathrm{GeV} / c$ for $\pi^{-} / \pi^{+}, K^{-} / K^{+}$ and $K^{ \pm} / \pi^{ \pm}$produced in MIPP NuMI data and the MINOS models . 100

6.5 The ratios as a function of $p_{z}$ at $0.2 \mathrm{GeV} / c<p_{T}<0.4 \mathrm{GeV} / c$ for $\pi^{-} / \pi^{+}, K^{-} / K^{+}$and $K^{ \pm} / \pi^{ \pm}$produced in MIPP NuMI data and the MINOS models .................... . . 101 


\section{List of Tables}

3.1 Hadronic Calorimeter specifications . . . . . . . . . . . . . 31

5.1 Binning scheme in $p_{z}$ and $p_{T} \ldots \ldots . \ldots . \ldots . \ldots 61$

5.2 The $\chi^{2}$ between data and Monte Carlo for $\pi^{ \pm}, K^{ \pm}$and $p^{ \pm}$before and after iteration ........................ 81

6.1 The $\pi^{-} / \pi^{+}$ratio in $\mathrm{p}+\mathrm{NuMI}$ interactions in $\left(p_{z}, p_{T}\right)$ at $120 \mathrm{GeV} / c . .92$

6.2 The $K^{-} / K^{+}$ratio in $\mathrm{p}+\mathrm{NuMI}$ interactions in $\left(p_{z}, p_{T}\right)$ at $120 \mathrm{GeV} / c .93$

6.3 The $K^{+} / \pi^{+}$ratio in $\mathrm{p}+\mathrm{NuMI}$ interactions in $\left(p_{z}, p_{T}\right)$ at $120 \mathrm{GeV} / c .94$

6.4 The $K^{-} / \pi^{-}$ratio in $\mathrm{p}+\mathrm{NuMI}$ interactions in $\left(p_{z}, p_{T}\right)$ at $120 \mathrm{GeV} / c .95$ 


\section{Acknowledgments}

After seven years of doctoral work, I have accumulated a wide range of experiences that I will be able to draw upon for many years to come. As well as learning much about physics from working at the forefront of the field, I have been exposed to the human interactions that are part of any major endeavor.

My advisor, Gary Feldman, gave me the opportunity to explore my interest in physics. His wisdom, intuition and friendliness have been a source of inspiration, particularly during the final few months of my $\mathrm{PhD}$. It has also been my pleasure to work with all the members of the MIPP and MINOS collaborations. In particular, from the MINOS collaboration, I would like to thank Zarko Pavlovic for his help and his extensive explanations of the Monte Carlo model that he built. I would also like to thank the neutrino group at Harvard for making my time in the department more enjoyable.

Our Graduate Programs Administrator Sheila Ferguson has been very supportive and caring throughout my graduate life. She has shared both my joy and my sadness. I must also thank our Laboratory Coordinator Robyn Simpson for her day-to-day help in the department.

From the non-physics world, I would like to thank the staff from the GSAS Facilities Office, the GSAS Housing Office and the GSAS Student Services, who have been like a second family to me; Bob Byrne has encouraged me to bake from scratch; Jill Larson has spent many an afternoon chatting with me over tea; Sheila Nazzaro and Patty Collyer have always been there to support me with their kindness and hu- 
mor; Garth McCavana and Ellen Fox provided me with much needed academic and non-academic advice. I shall miss them all greatly.

There are countless others who have been there for me throughout my time as a graduate student. From Harvard, Hanley Chiang and Elaine Gee stand out as good caring listeners, and Malathi Thothathiri even supported me during my thesis defense! From my undergraduate days, Chunmin Chen has been a wonderful friend; hunting down and enjoying delicious food in the Boston area and watching movies with her has become one of my favorite pastimes.

My parents allowed me to choose an academic path, and always provided unlimited support and love despite not always understanding my research! My brother Thomas inspires me with his constant stream of ideas about almost anything in the world. My grandmother is the strongest woman I know. Though she would prefer me to be stay at home in Hong Kong, she has never, and would never, try to prevent me from pursuing my interests. At this time, I think about those that are no longer with us: my grandfather and my uncle. My grandfather passed away before I started my $\mathrm{PhD}$ and I hope this achievement will make him proud. My uncle taught me how to ride a bike and was always curious about the latest electronic gadgets - I think his enthusiasm rubbed off on me. It was tragic that he had to pass away only a month before my graduation.

Finally, Christopher Smith has been my guiding light and love. Over the past years, we have spent countless hours in transatlantic phone conversations. He has 
been my best colleague and my best friend, from discussing physics ideas to providing emotional support. He kept me on the right path even during the hardest times, and when I felt hopeless, he continued to have faith and belief in me. 


\section{Chapter 1}

\section{Introduction}

The study of proton interactions with different atomic targets represents the central goal of the Main Injector Particle Production (MIPP) experimental programme which is aimed at a comprehensive study of hadronic interactions at various energies. The MIPP detector layout combines wide acceptance coverage with complete particle identification, the aim being to collect large statistics data samples for all interaction types. This provides the basis for a model independent understanding of the underlying production mechanisms.

The MIPP experiment measures the secondary hadron production spectra in different momentum regions, and the present study addresses the pion/kaon ratio in $120 \mathrm{GeV} / c$ proton on NuMI target interactions for momenta above $20 \mathrm{GeV} / c$. The motivation for these measurements comes from long-baseline neutrino oscillation experiments, such as MINOS (Main Injector Neutrino Oscillation Search) [2], which use 
proton-target collisions to produce a well understood neutrino beam. The neutrinos in these experiments originate as the decay products of mesons (mainly pions and kaons) or of muons, which themselves are primarily pion or kaon decay products. Thus, understanding the detailed composition of accelerator neutrino beams relies heavily on understanding the nature of the hadronic cascade, i.e. the production spectra of the secondaries resulting from the collision of a high energy particle.

The available experimental data on this topic are presently not extensive. The precision of the MINOS experiment depends on the accuracy of the predictions of the neutrino fluxes at the Far detector based on the observations in the Near detector. This extrapolation of the neutrino energy spectrum is a strong function of the details of the production spectra of the secondary pions and kaons. The MIPP Experiment therefore aims to provide high precision reference data for the experiment.

The layout of this thesis is arranged as follows: previous measurements are discussed in Chapter 2. In Chapter 3, the MIPP experimental setup at Fermilab is described. Chapters 4 outlines several key reconstruction stages which are important for this analysis. Chapter 5 describes the NuMI target analysis, the results of which are presented and discussed in Chapter 6 . 


\section{Chapter 2}

\section{Particle Production}

One of the largest uncertainties in the extrapolation of the Near detector neutrino spectra to the Far detector site in MINOS arises from hadron production modeling of the NuMI target. This chapter reviews the available data and its relevance to the NuMI beam. The uncertainties in the absolute prediction and the relative Far-Near spectra due to uncertainties in hadron production are also estimated.

\subsection{Particle Production Data from Related Exper- iments}

The prediction of secondary production on the NuMI target has large uncertainties primarily due to the lack of available data relevant to the NuMI case. The most relevant measurements are as follows: Atherton et al[6], Barton et al[7] and SPY [8]. 
Figure 2.1 shows the $p$ and $p_{T}$ distribution of $\pi$ at the target weighted by the number of predicted neutrino interactions produced at the MINOS Near and Far detectors for the low energy beam. Superimposed on the $p$ and $p_{T}$ distributions are points showing where previous measurements have been made. While the SPY data cover a reasonable fraction of the peak region $(0<\mathrm{p}<10 \mathrm{GeV} / \mathrm{c})$, the hadron production data in the high energy tail $(p>20 \mathrm{GeV} / c)$ are significantly more sparse.

\section{Low Energy Beam}

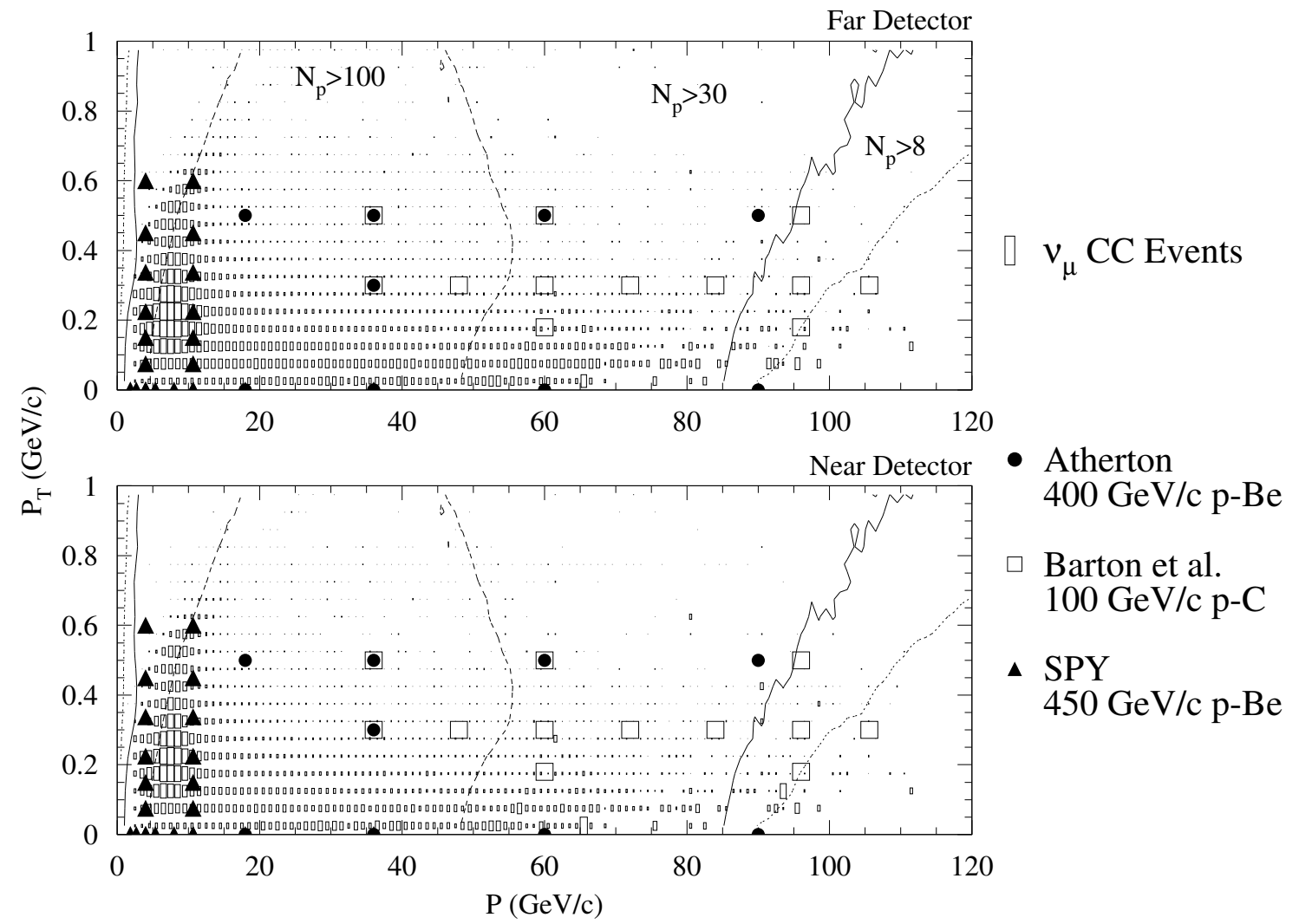

Figure 2.1: The distribution in $p$ and $p_{T}$ for secondary pions produced on the NuMI target. Secondaries have been weighted by their contributions to the neutrino event rate at the Far (top) and Near (bottom) detectors. Overlaid are the locations of existing hadron production measurements [9].

Most of the available data was taken using Beryllium targets at significantly higher 
primary momentum and on shorter targets than that used by MINOS. The data must be extrapolated from different target nuclei and beam momentum to be applied to the NuMI case. For these reasons, roughly 20-30\% uncertainties in the predictions of the secondary production of protons from the NuMI target are introduced.

\subsection{Simulations of Particle Production from the NuMI Target and Uncertainties in the NuMI \\ Neutrino Spectra}

To estimate the impact of uncertainties in hadron production, simulations and parameterizations of the NuMI target have been made [9]. Figure 2.2 compares the predictions of the MINOS absolute neutrino event rates at the Near detector for four hadron production models of the NuMI target: GFLUKA (GEANT-FLUKA $[10,11,12])$, MARS $[13,14,15,16]$, BMPT [17] and Malensek [18]. Variations in the absolute predictions are roughly 10-25\%.

The extrapolation of the Near spectrum to the Far spectrum is not straightforward. The distance to the Far detector is roughly 1000 times larger than the length of the NuMI beamline. Thus, to a very good approximation the Far detector sees a point neutrino source. However, the Near detector sees an extended source of neutrinos. Figure 2.3 shows the estimated ratio of the Far neutrino flux to the Near neutrino flux for the same four models. The predictions differ by as much as $20 \%$ in the Far/Near 

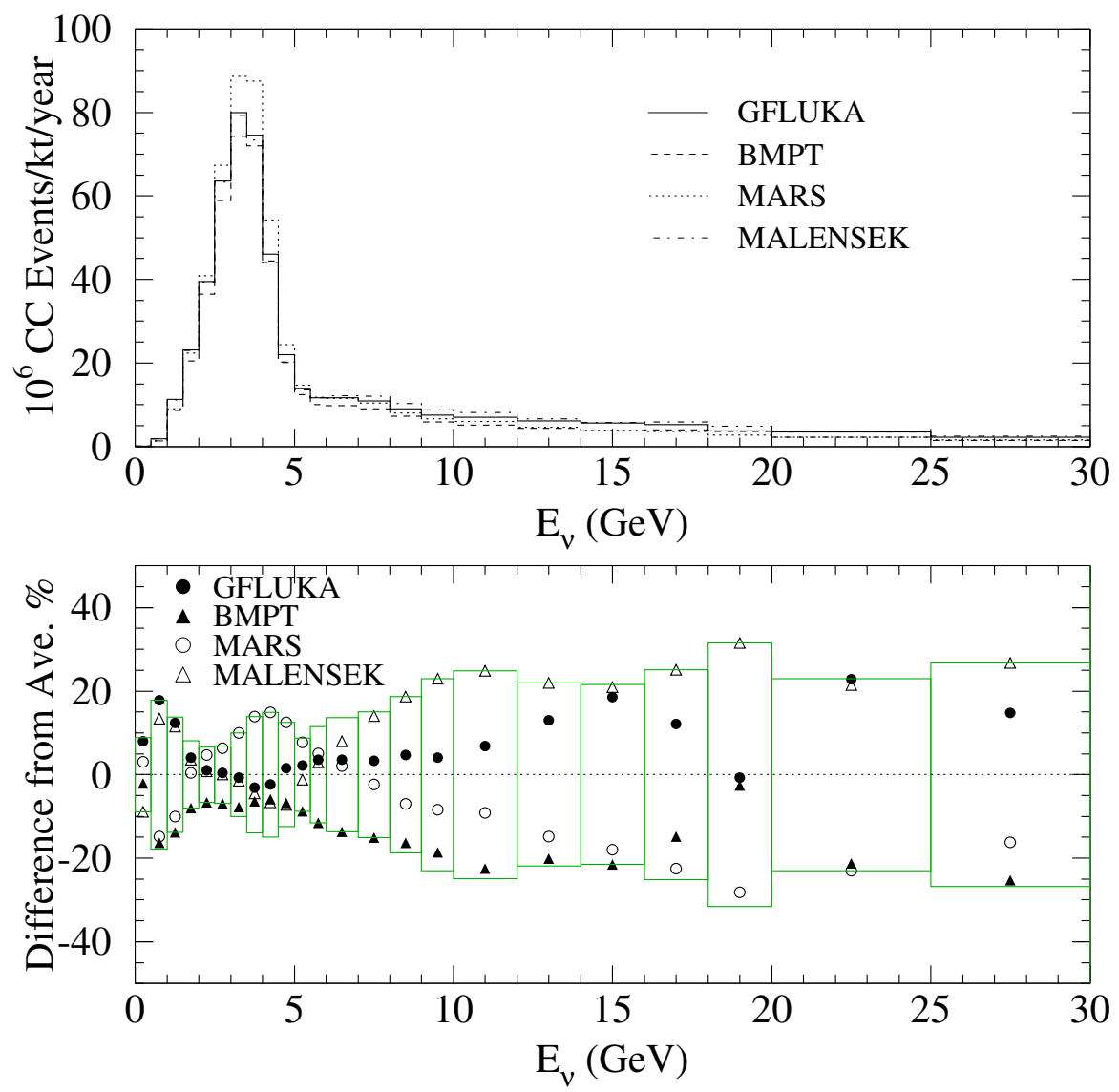

Figure 2.2: Predictions of the absolute neutrino rates at the MINOS Near detector using four hadron production models [9].

ratio.

The MINOS experiment will compare the neutrino spectrum at the Far detector to the expected spectrum based on the measurement of the spectrum at the Near detector. Therefore, a reduction in the uncertainty in the Far/Near ratio due to hadron production would benefit the MINOS results. 

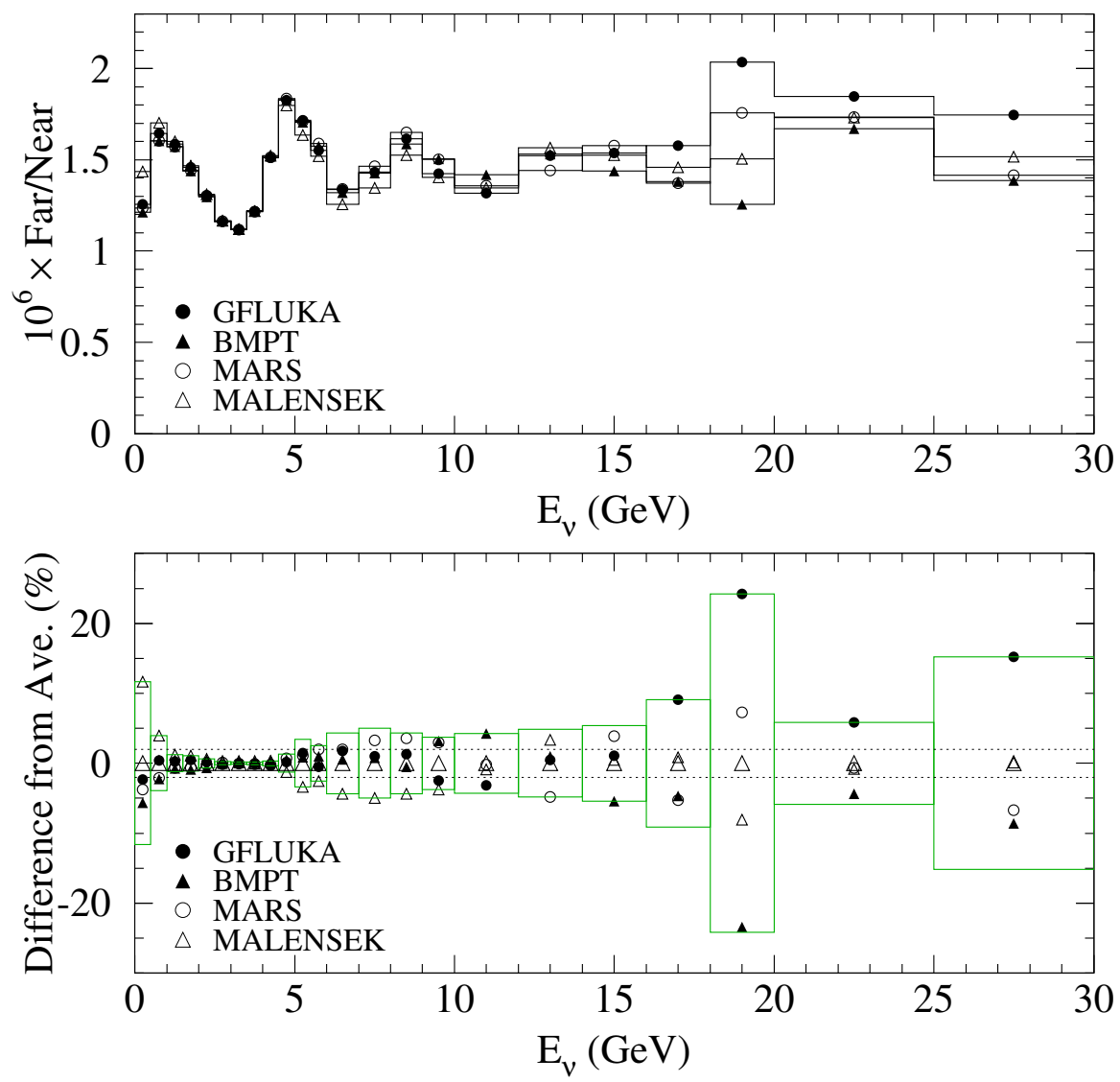

Figure 2.3: Predictions of the ratio of the Far neutrino flux to the Near neutrino flux using four hadron production models [9]. 


\section{Chapter 3}

\section{The MIPP Experiment}

The Fermilab experiment E907: the Main Injector Particle Production (MIPP) Experiment took fixed target data in the Meson Center Beam Enclosure 7 (MC7) beamline of the Meson Area at Fermilab from summer 2004 until the beginning of 2006. The experiment is designed to perform high statistics studies of hadron-nucleus interactions at various momenta $(5-120 \mathrm{GeV} / c)$ for target nuclei ranging from Hydrogen to Bismuth. It uses the $120 \mathrm{GeV} / c$ proton beam from the Main Injector and measures particle production from primary beam interactions in the NuMI/MINOS target and primary and secondary beam interactions in a variety of thin targets. This data has applications in many fields of physics including neutrino beams, atmospheric neutrinos, nuclear physics, heavy ion physics, and proton radiography. The focus of this thesis is the analysis of data from the NuMI target running.

In the following sections, the details of the beamline and the detectors are de- 
scribed.

\subsection{The Beamline}

MIPP runs in the Meson Center beamline. The experiment consists of a secondary beamline containing a beam transport pipe and a set of of Cherenkov counters. A picture of the physical layout of the MIPP beamline is given in Figure 3.1.

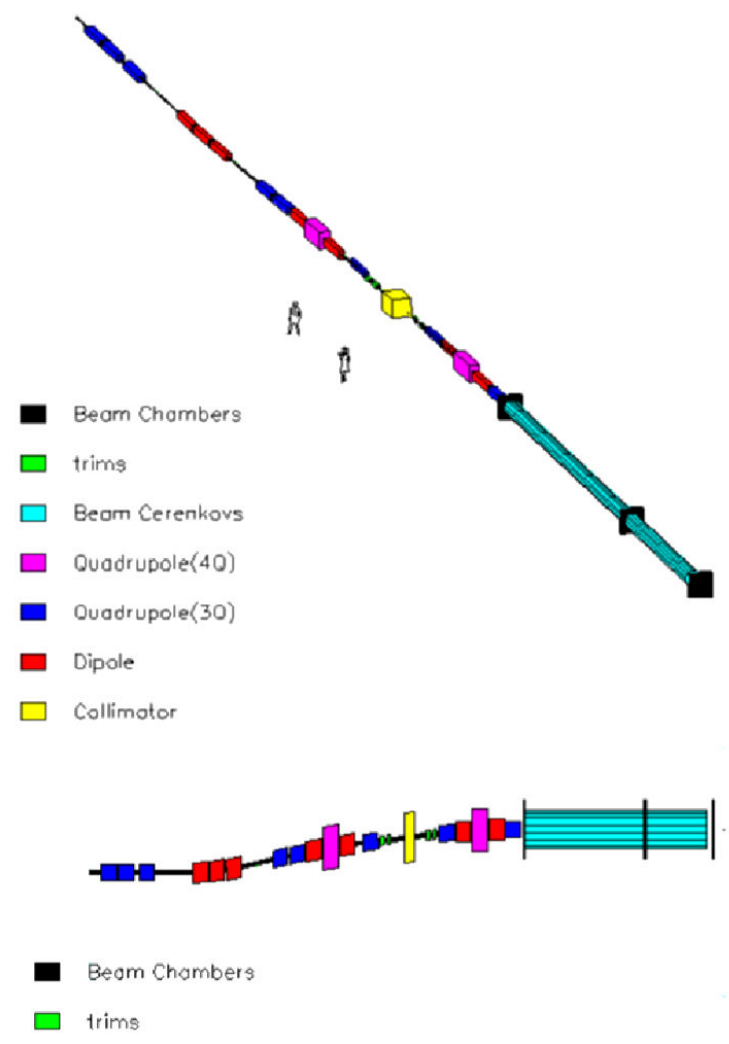

Figure 3.1: The elements and an elevation view of the MIPP beamline.

Primary data are acquired at $120 \mathrm{GeV} / c$ by transporting a low intensity beam from the Main Injector directly onto the NuMI target. The beam flux is limited by 
the data taking rate of the experiment. The Meson Center beamline also serves to provide a secondary beam of $\pi^{ \pm}, K^{ \pm}$, and $p^{ \pm}$particles to the MIPP experiment. The secondary beam momenta range from $5 \mathrm{GeV} / c$ to $90 \mathrm{GeV} / c$. The incident secondary beam species are tagged by threshold beam Cherenkov counters upstream of the secondary target.

\subsubsection{Primary Beamline}

The primary beamline comes directly from the Main Injector. Control over primary beam characteristics is established through a focusing quadrupole doublet and adjustable collimators located upstream of the primary target. Due to enclosure restrictions and data taking rates in the Time Projection Chamber (TPC, to be described in Subsection 3.3.1), intensity on the primary beam was attenuated to less than $10^{5}$ particles per second. By tuning the primary optics and the collimator aperture, the desired primary beam intensity was achieved. During the NuMI target running period, it was necessary to reduce the beam intensity to $10^{3}$ particles/spill, with a 4-second spill occurring every two minutes from the Main Injector. This is because the NuMI target is two-interaction lengths long and so $90 \%$ of the incident particles interact with the target. The primary beam intensity had to be maintained at a lower rate so that the desired event rate was achieved thus avoiding pile-ups. 


\subsubsection{Secondary Beamline}

Using protons from the Meson Center beamline, a secondary beam of charged $\pi$ 's and K's is produced. The $\pi$ 's and K's impinge on a secondary target - the scattering on the secondary target being the focus of the experiment. The secondary beamline is capable of selecting and transporting a beam with an energy in the range of $5-90 \mathrm{GeV} / c$. The secondary beamline must accommodate both the demanded momentum resolution and the precision in particle identification. The former requires the momentum-dispersed beam size to be larger than the transverse beam size and the latter requires low divergence at the Cherenkov detectors. A series of dipoles performs the momentum selection followed by the Cherenkov counters which identify the beam particles.

The primary target is a $0.5 \times 0.5 \times 20 \mathrm{~cm}$ copper block, located in between four dipole magnets. The first two dipoles bend the beam onto the primary target in MC5, and the latter two dipoles bend the beam back onto the beam center line for the Meson Center. In addition, three quadrupoles downstream of the primary target focus the beam onto a jaw collimator, with a vertical aperture which can be varied. The strength of the downstream dipoles determines the central momentum value of the beam, and the momentum spread, $d p / p$, is then set by the jaw aperture size. Finally, the beam is refocused onto the E907 experimental target by three additional quadrupoles. 


\subsubsection{Beam Cherenkov}

The two Beam Cherenkov counters are long cylinders with heads at the exit of the counters. Each counter is read out through two PMTs - the inner PMT, which is covered by a second mirror with an aperture, and the outer PMT. The Cherenkov light from different particle types is reflected from the focusing mirror on the head. The reflected light below the cutoff angle is focused into the second mirror. The light that passes through the aperture of the second mirror is detected by the the inner photomultiplier. The reflected light above the cutoff angle is detected by the outer photomultiplier. The cutoff Cherenkov angle for the upstream counter is 5 mrad and the angle for the downstream counter is $7 \mathrm{mrad}$. The purpose of these counters is to identify the three possible beam particle species - protons, kaons, and pions. One counter is used to separate pions from kaons, and another is used to separate kaons from protons. The particles are all at the same momentum and that momentum ranges from 5 to $90 \mathrm{GeV} / c$.

For each threshold Cherenkov detector, one particle is below threshold and one is above. The lengths of the detectors are determined by the mass difference of the particles which need to be differentiated and the momentum at which the differentiation is made. The long detector is $22.9 \mathrm{~m}$ (to separate the pions from kaons) and the shorter one is $12.2 \mathrm{~m}$ long (to separate the protons from kaons), so they yield the same number of photoelectrons in the worst case (high momentum).

At specific pressure, the protons are below threshold in the upstream counter, 
and the kaons and the pions are above the threshold and emit light in both. The Cherenkov light from the kaons are detected mostly by the inner PMT of the upstream counter and the outer PMT of the downstream counter. The light from the pions is incident on the outer PMT of the upstream counter, and the light from the protons focus onto the inner PMT of the downstream counter. Using two threshold Cherenkov detectors, one can then determine which particle passed, by looking for which PMTs get hit by Cherenkov light.

For the case of pion/kaon separation, the required momentum range can be accommodated by using Nitrogen gas. Also, the index of refraction is changed by varying the gas pressure so that the Cherenkov light of selected particles goes through the aperture and hits the inner phototube. The Cherenkov light from particles with masses different from those of the detected particle hit the outer phototube. In the case of the proton/kaon separation, the same gas is used for most of the momentum range. At low momenta (below $30 \mathrm{GeV} / c$ ) a larger index of refraction than Nitrogen is required. $\mathrm{C}_{4} \mathrm{~F}_{10}$ is used with maximum pressure to be set at about 3 atm. The lower momentum beams $(5 \mathrm{GeV} / c)$ can be separated by Time-Of-Flight instead of using a high index of refraction gas.

\subsection{Targets}

The MIPP experiment uses three different types of target: nuclear targets, a cryogenic target and the NuMI target. 
The production thin nuclear targets are mounted on a target wheel, which was built by the University of Colorado and taken to Fermilab unassembled. It contains 6 slots, only four of which are occupied at any one time. The targets employed are Beryllium, Carbon 1\%, Carbon 2\%, Aluminum, Copper, Silver and Bismuth. In the order given, the targets are $0.94,0.57,0.94,0.992,0.973,1.4$ and 0.87 interaction lengths. The two vacant slots were used for background measurements. The wheel is not centered on the beam, since the target holes are off-axis. The wheel is turned by an ACNET (Fermilab Accelerator Control NETwork) controlled motor mounted to the Time Projection Chamber (TPC) table upstream of the TPC, and outside of the magnetic field of the "Jolly Green Giant" (to be described in Subsection 3.3.7).

The cryogenic target is used to run with liquid hydrogen, and requires a special setup.

MIPP used the spare NuMI target from MINOS, which is shown in Figure 3.2. The target core constitutes 47 graphite segments soldered to two stainless steel cooling pipes with an external diameter of $6.0 \mathrm{~mm}$ and thickness of $0.4 \mathrm{~mm}$. Each graphite segment is $0.64 \mathrm{~cm}$ wide, $2.0 \mathrm{~cm}$ tall and $1.36 \mathrm{~cm}$ long with a $0.67 \mathrm{~cm}$ gap in between segments. The gaps between the segments allow most of the produced pions and kaons to leave the target before interacting and avoid contact of heated segments. The target core is inserted into a $0.4 \mathrm{~mm}$ thick, $30 \mathrm{~mm}$ diameter Aluminum cylinder casing. The total length of the casing is about $1.2 \mathrm{~m}$ long.

The NuMI target is made from carbon and almost 2 nuclear interaction lengths. 


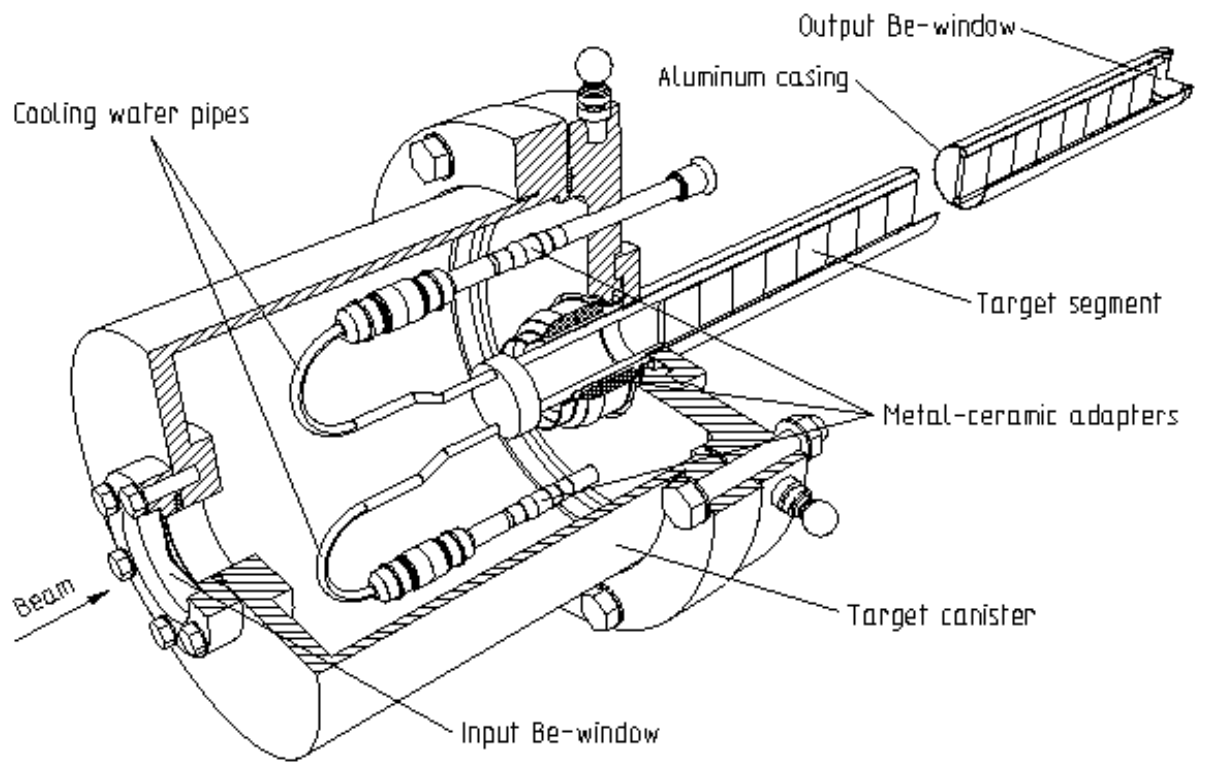

Figure 3.2: Schematic of the NuMI target design [19].

This leads to $\sim 90 \%$ of the incoming protons to interact within the target, thus reducing the backgrounds created by non-interacting protons and maximizing meson production.

The pair of stainless steel cooling tubes were designed to remove heat deposited in the target in MINOS. Since the beam intensity during MIPP NuMI running period was much lower than MINOS, no water was circulated in the tubes. And this was taken into account in the MIPP Monte Carlo simulation, which is described in Section 4.1. 


\subsection{The Detectors}

MIPP is a low-cost experiment which uses existing hardware to measure and identify a range of particles over a wide momentum range. The perspective layout of the MIPP experiment is shown in Figure 3.3 with the beam coming from the upper left, and Figure 3.4 shows a plan view of the MIPP detectors with a simulated shower in the Electromagnetic Calorimeter (EMCAL) and the Hadronic Calorimeter (HCAL). The sub-detectors are reused from previous Fermilab and Brookhaven fixed target experiments. The experiment uses a Time Projection Chamber (TPC) most recently used in the Brookhaven E910 experiment to provide vertex and track reconstruction [20]. The TPC also provides particle identification at the lowest particle momenta $(p<0.7 \mathrm{GeV} / c)$. The TPC is placed in a large magnet (the "Jolly Green Giant", JGG) which provides a field of $7 \mathrm{kG}$. Following the TPC, a threshold Cherenkov detector (also from E910) provides particle identification in the momentum range from 3 to $17 \mathrm{GeV} / c$. A Time-Of-Flight (TOF) system is used to fill in the gap in particle identification between $0.7 \mathrm{GeV} / c$ and $3 \mathrm{GeV} / c$. The TOF is designed to have a $200 \mathrm{ps}$ timing resolution, providing $2.5 \sigma$ particle identification separation at $2 \mathrm{GeV} / c$. Then follows the ROSIE spectrometer magnet (most recently used in the DONUT experiment [21]), which provides a $10 \mathrm{kG}$ magnetic field. Multi-wire proportional and drift chambers, taken from the SELEX [22] experiment and the E690 [23] experiment respectively, are interspersed throughout the apparatus. These are used in addition to the TPC for particle tracking. Downstream of ROSIE and 
the wire chambers is a Ring Imaging Cherenkov detector (RICH). Above $17 \mathrm{GeV}$, particle identification is provided by the RICH, which was originally built for the SELEX experiment $[24,25,26]$ at Fermilab. This detector is of particular importance to the NuMI target measurement as the momentum range above $10 \mathrm{GeV}$ is the most relevant for the MINOS neutrino fluxes. The final detectors in the chain are an electromagnetic calorimeter and a hadronic calorimeter.

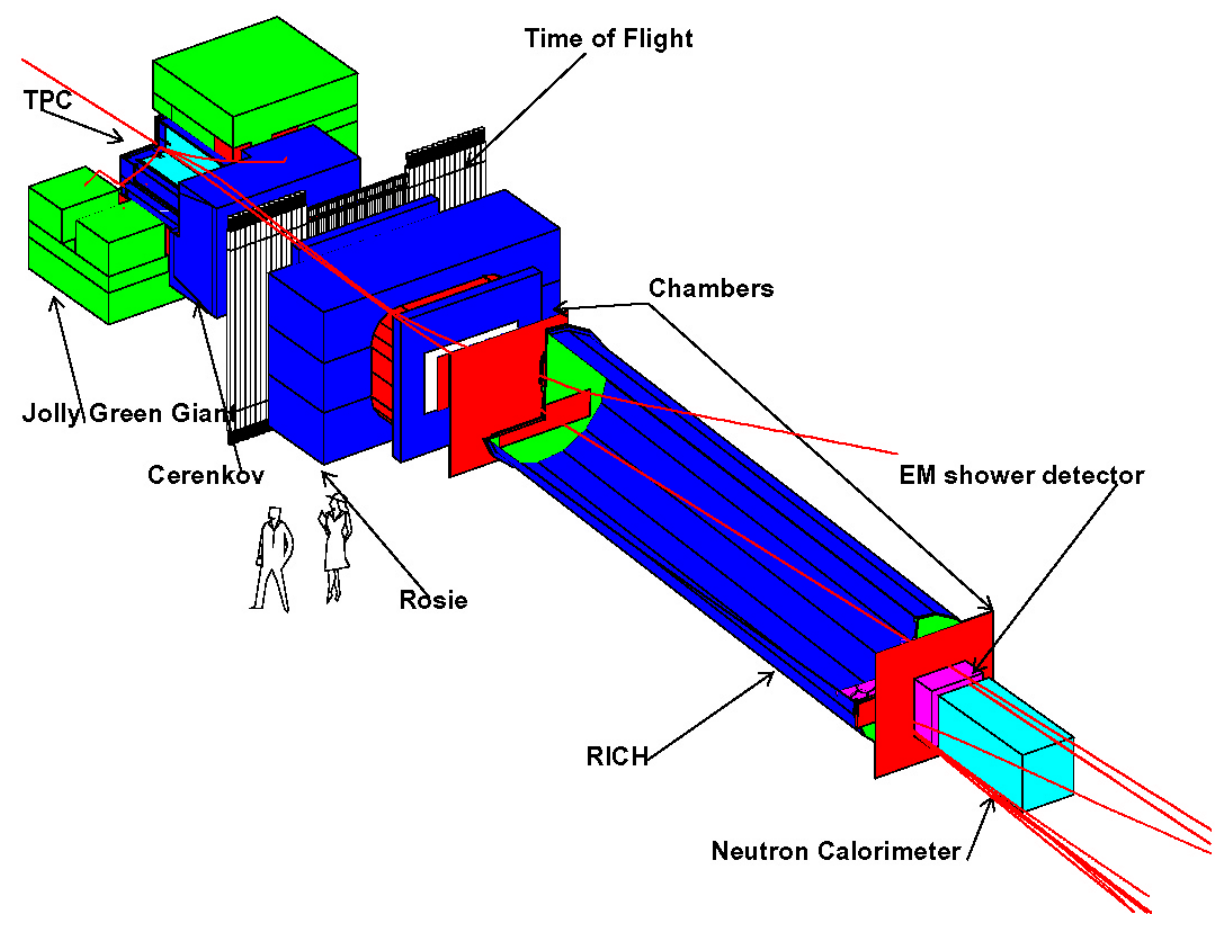

Figure 3.3: Layout of the MIPP experiment.

\subsubsection{Time Projection Chamber}

One of the most important detectors in the MIPP Experiment is the Time Projection Chamber (TPC), operated with P10 (90\% Ar, 10\% $\left.\mathrm{CH}_{4}\right)$ gas, a -10 kV drift 

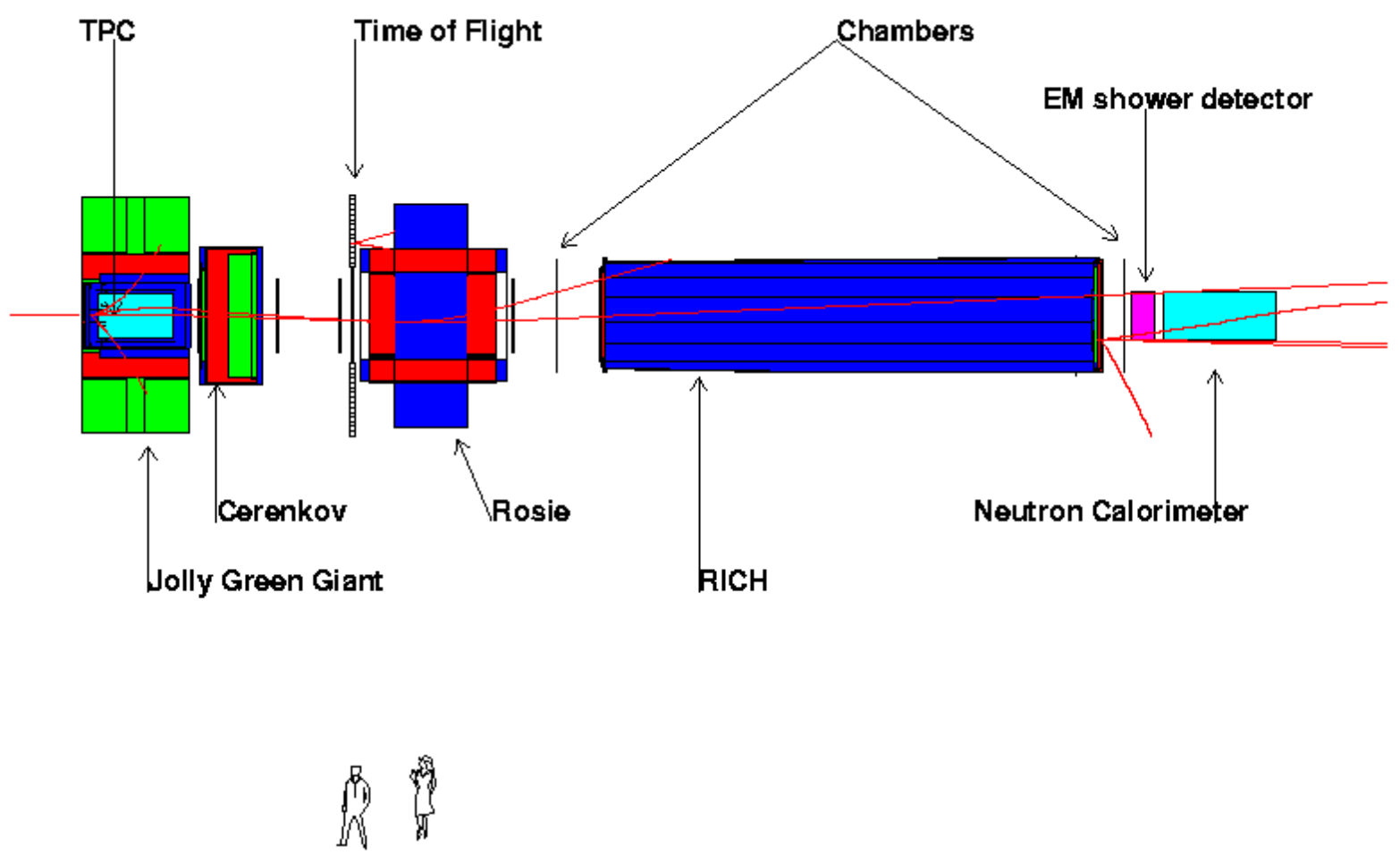

Figure 3.4: Plan view of the E907 detectors.

potential, and $+1,300 \mathrm{~V}$ anode potential for gas gain of the ionization signal. The TPC was built by Lawrence Berkeley National Laboratory (LBL) for the EOS experiment at the Bevalac $[27,28,29,30,31,32,33]$. Figure 3.5 shows an exploded view of the chamber with the outer case removed.

The TPC provides true 3D tracking in the magnetic field for most of the particles exiting on the downstream side of the target. The active tracking region in the TPC is a rectangular box $1.5 \mathrm{~m}$ long in the beam direction, $96 \mathrm{~cm}$ wide in the bending direction and $75 \mathrm{~cm}$ high in the drift direction, with a uniform electric field of $120 \mathrm{~V} / \mathrm{cm}$. The beam passes directly through the center of the TPC volume. The 


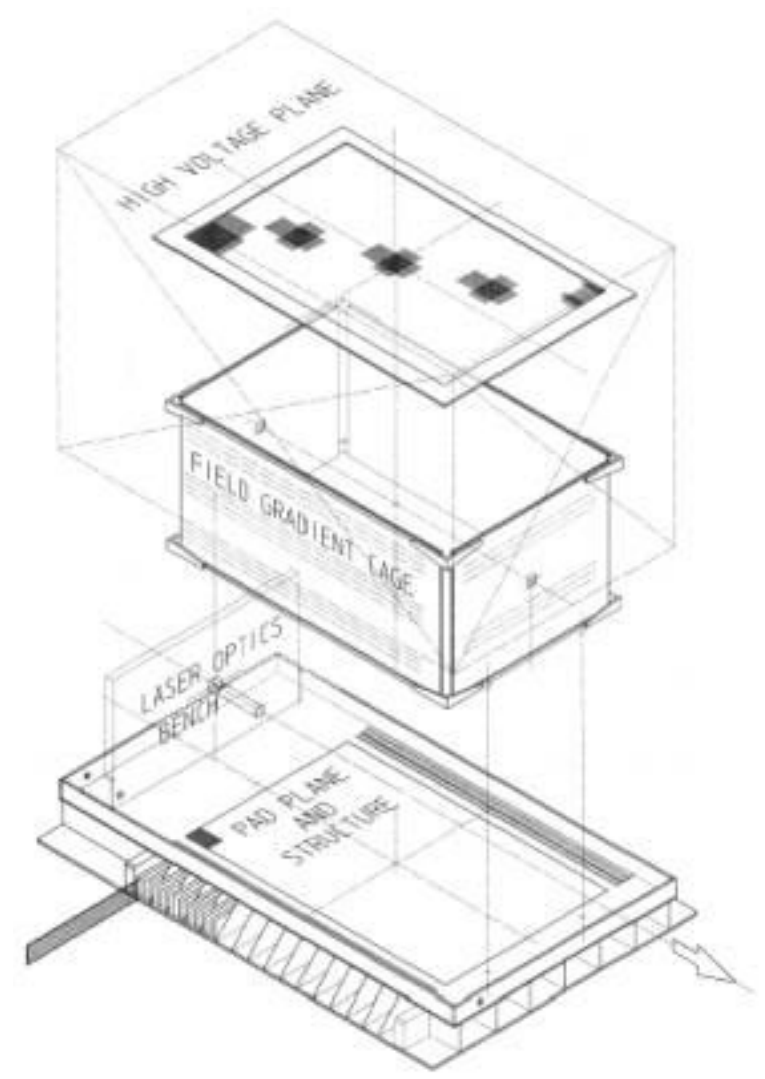

Figure 3.5: Exploded view of the TPC with the outer case removed. The active volume is defined by the pad plane, field cage, and high voltage (cathode) plane [27].

tracking in the magnetic field of $7 \mathrm{kG}$ measures particle rigidity and multi-sampling of $\mathrm{dE} / \mathrm{dx}$ along the track provides particle identification.

The TPC was configured as a drift volume enclosed with a rectangular field cage sitting over a single, multi-wire proportional chamber (MWPC) - pad plane structure. Charged particles passing through the ground array are accelerated into anode wires. The electrons drift down under the influence of a uniform electric field set up by the cathode plane and ground wire array (Figure 3.6). The electrons pass through the ground array and are accelerated into anode wires. The electron-gas scattering that 
occurs in the high field region around the anode wires causes additional ionization. The ionization induces image charges in the pads, which are amplified and digitized by the MWPC and recorded as a function of time. The MWPC pad plane provides a full $2 \mathrm{D}$ readout and the drift time provides the third dimension. The MWPC pad system is composed of $20 \mu \mathrm{m}$ anode wires on a $4 \mathrm{~mm}$ pitch located $4 \mathrm{~mm}$ above a single panel, which is a continuous rectangular array of $8 \mathrm{~mm}$ by $12 \mathrm{~mm}$ pads $(15,360$ pads total). The electrons create avalanches as they drift to the anode wires. The positive ions created in the avalanche induce signals in the pads as they drift from the anode region. The induced signal is spread over 2 to 3 pads allowing accurate position determination through reconstruction. The TPC volume is divided up into a 2.5 million pixel volume (128 pad rows $\times 120$ pads $\times 160$ time buckets). The drift velocity is $5 \mathrm{~cm} / \mu \mathrm{s}$.

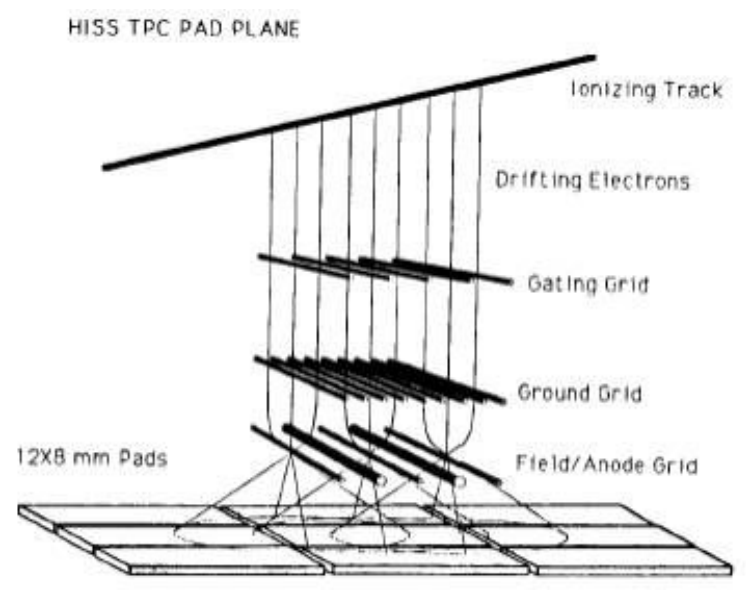

Figure 3.6: Sketch of the wire planes and field lines [27].

The 15,360 pads are read out via custom original electronics and a VME interface 
to commercially available data acquisition processors. The signals are written at $10 \mathrm{MHz}$ and they are readout and digitized at a low rate with a commercial 12 bit ADC chip. Our data was recorded in 100 ns time buckets.

\subsubsection{Time-Of-Flight}

The TOF wall is located immediately before the ROSIE magnet and consists of scintillator with R5900U phototubes. This system measures the time of flight for the particles between the target and the scintillator. The size of the TOF is $3.5 \mathrm{~m}$ wide by $3 \mathrm{~m}$ high. It has $543 \mathrm{~m}$ long scintillator bars, with $405 \mathrm{~cm} \times 5 \mathrm{~cm}$ bars at the center and the sides covered by $10 \mathrm{~cm} \times 10 \mathrm{~cm}$. The TOF has the ability for particle identification between $1 \mathrm{GeV}$ and $3 \mathrm{GeV}$, and it achieves 180 ps resolution on beam tracks. However, due to the trigger, a 150 ns delay cable was inserted. This caused variations up to $2 \mathrm{~ns}$ due to temperature changes in the experimental hall.

\subsubsection{Cherenkov}

The Cherenkov detector, shown in Figure 3.7(a), is located immediately downstream of the first drift chamber. It is used to identify pions, kaons and protons. The gas radiator, $\mathrm{C}_{4} \mathrm{~F}_{10}$, has a low threshold momentum of $2.6 \mathrm{GeV} / c$ for charged pions, and $17 \mathrm{GeV} / c$ for protons.

The box dimensions are $\mathrm{W} \times \mathrm{H} \times \mathrm{D}: 2.8194 \mathrm{~m} \times 3.3274 \mathrm{~m} \times 1.143 \mathrm{~m}$. The size of the front aperture is $139.7 \mathrm{~cm} \times 190.5 \mathrm{~cm}$. There are 96 primary mirrors arrayed into 


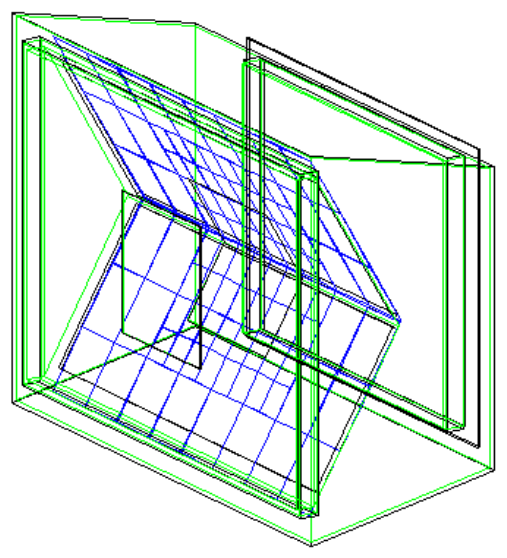

(a) A schematic view.

\begin{tabular}{|c|c|c|c|c|c|c|c|}
\hline 35 & SI & 89 & 20 & $\Delta_{1}$ & $\Delta_{2}$ & 45 & 48 \\
\hline 324 & 37 & \begin{tabular}{l|l}
4 & 6 \\
3 & 7
\end{tabular} & \begin{tabular}{|l|l|l|}
12 & 18 \\
11 & 15
\end{tabular} & $\begin{array}{ll}5 \\
5\end{array}$ & 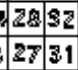 & 24 & 47 \\
\hline$s 3$ & 36 & \begin{tabular}{|l|l}
2 & 6 \\
1 & 5 \\
\end{tabular} & \begin{tabular}{|l|l}
10 & 12 \\
9 & 19
\end{tabular} & 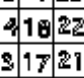 & & 43 & $\Delta_{6}$ \\
\hline ง1 & gst & 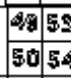 & $\frac{57 / 37}{51}$ & $\begin{array}{l}78585 \\
268: 70\end{array}$ & \begin{tabular}{|l|}
7897 \\
79478
\end{tabular} & $\$ 1$ & 52 \\
\hline Ez & 95 & \begin{tabular}{|l|l|l}
51 & 5.5 \\
5 & 52 & 56
\end{tabular} & 5 & $\begin{array}{l}38771 \\
48672\end{array}$ & \begin{tabular}{|l|}
75579 \\
75690
\end{tabular} & 92 & 95 \\
\hline 8s & $s_{6}$ & 07 & 88 & 89 & 90 & 98 & פ6 \\
\hline
\end{tabular}

(b) The mirror numbering when look-

ing at the front of the counter, with

beam heading into the screen.

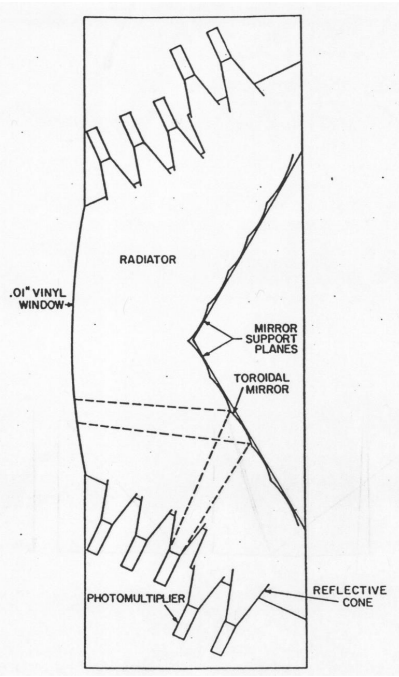

(c) Side View.

Figure 3.7: The Cherenkov detector. 
two mosaics assembled on upper and lower panels. The mirrors come in three sizes: $6 " \times 6.928 ", 12 " \times 13.856 "$ and $12 " \times 9.660 "$. The $6 "$ mirrors are $1 \mathrm{~mm}$ thick glass, while the others are $1.5 \mathrm{~mm}$ thick. These mirrors divide the common radiator volume into the 96 detection volumes giving the detector its segmentation. Each mirror is matched to an individual phototube. Smaller mirrors cover the central part of the counter where the particle densities are higher. The mirror numbering and size are shown in Figure 3.7(b).

The secondary mirrors are right angled cones, tipped such that the axis of symmetry is coincident with the reflected central ray from the target off of the appropriate primary mirror. The secondary mirrors reflect Cherenkov light onto the photocathodes of the photomultiplier tubes. The side view in Figure 3.7(c) shows the schematic of the Cherenkov and the arrangement of the optics.

The tubes are modified with a wavelength shifting coating on the photocathode window surface to increase the detection efficiency by shifting blue Cherenkov light to green wavelengths.

\subsubsection{Ring Imaging Cherenkov Detector}

The RICH detector $[24,25,26]$, pictured in Figure 3.8 uses a $32 \times 89$ array of $\frac{1}{2}$ inch photomultiplier tubes. An example event display is shown in Figure 3.9. The PMT array is used to measure the Cherenkov ring radius of particles traversing the gas volume. 


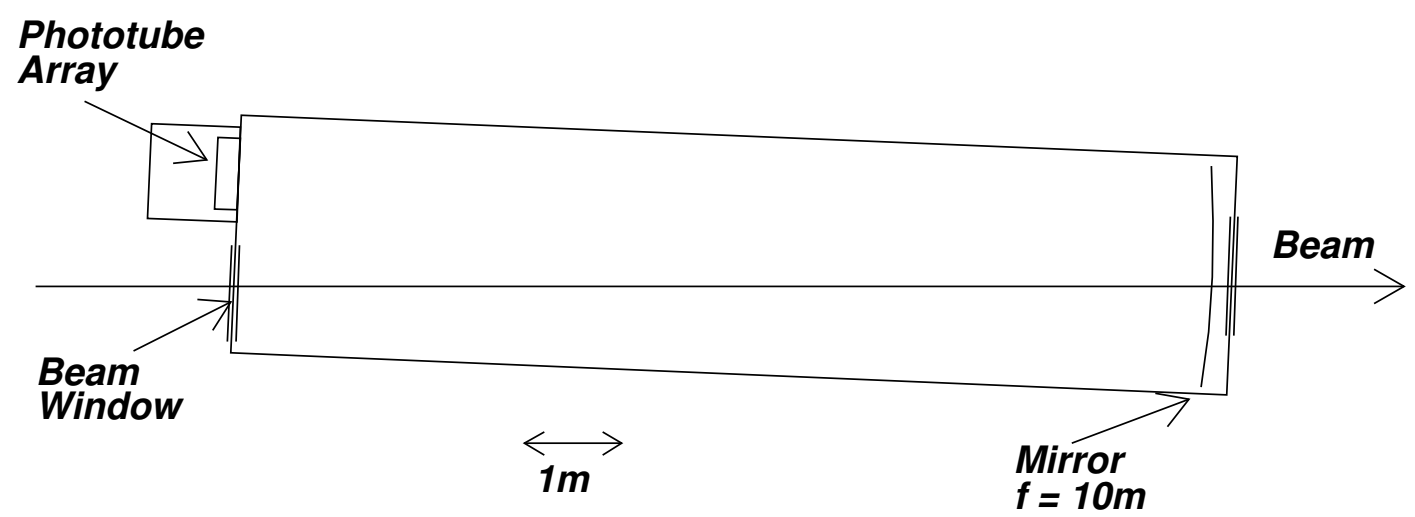

Figure 3.8: A schematic view of the RICH detector [25].

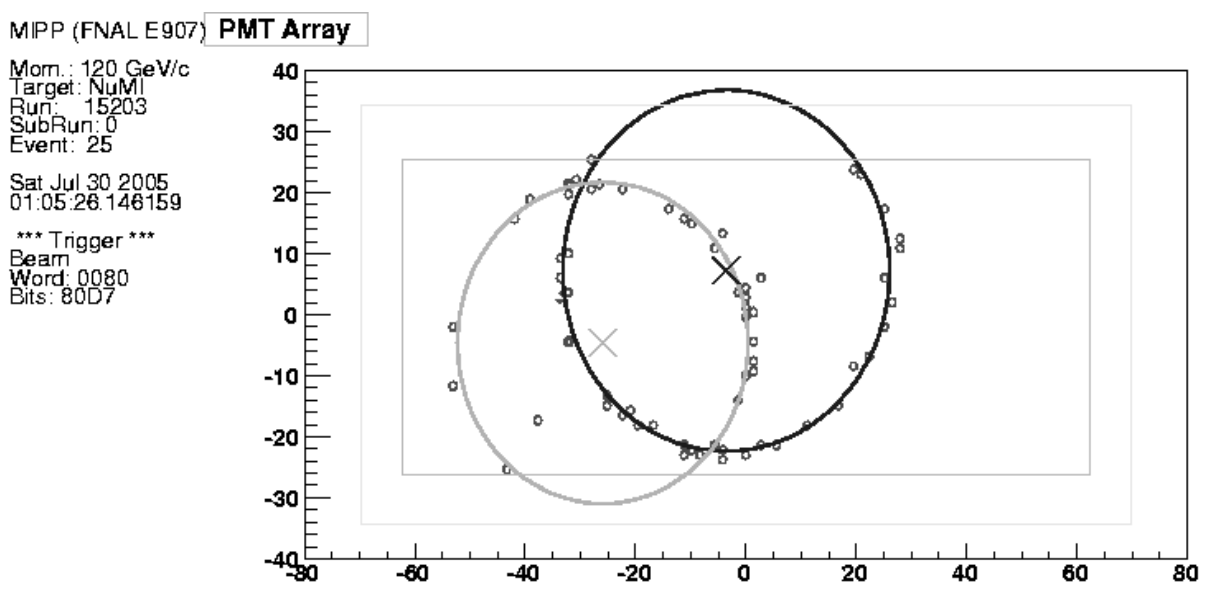

Figure 3.9: Online display in the RICH detector.

The main parts of the detector (vessel, mirrors, photon detectors) are briefly described below.

\section{Vessel and Gas System}

The RICH vessel is a low carbon steel radiator $10.22 \mathrm{~m}$ in length, $2.4 \mathrm{~m}$. in diameter and with a wall thickness of $\frac{1}{2}$ in, filled with Carbon Dioxide at about 
$1.03 \mathrm{~atm}$. The end flanges are $1.5 \mathrm{in}$. thick aluminum with provisions for thin beam windows and a phototube holder plate to be described later.

Provisions were made for thermistors at both ends of the vessel to monitor internal temperature and a LED pulser test array to supply calibration signals for the phototubes.

The vessel is tilted off the horizontal axis by $2.4^{\circ}$ with the beam entering horizontally through a thin window so that particles do not pass through the phototubes.

Over the course of a running period lasting for about a year the oxygen level was kept at 1000 parts per million. The volume of $\mathrm{CO}_{2}$ slowly leaks over time, so $\mathrm{CO}_{2}$ was added on a weekly basis to keep the pressure variation to within $1 \%$.

To monitor the status of the detector, the following parameters were logged: atmospheric pressure, vessel pressure, vessel upstream temperature, vessel downstream temperature, temperature at several locations within the phototube box, voltages at the end of the HV Zener diode chains, and status of the low voltage system.

\section{Mirrors}

The mirror plane at the end of the vessel consists of an array of 16 hexagonally spherical mirrors, as shown in Figure 3.10. The mirrors are mounted in an array which is approximately $2.4 \mathrm{~m}$ wide and $1.2 \mathrm{~m}$ high, fixed individually to a flat, low mass honeycomb panel of 1 in. thickness with a 3-point kinematic mount. Each mirror is $40 \mathrm{~cm}$ across (46 cm tip-to-tip), $1 \mathrm{~cm}$ thick, made of low expansion glass, 


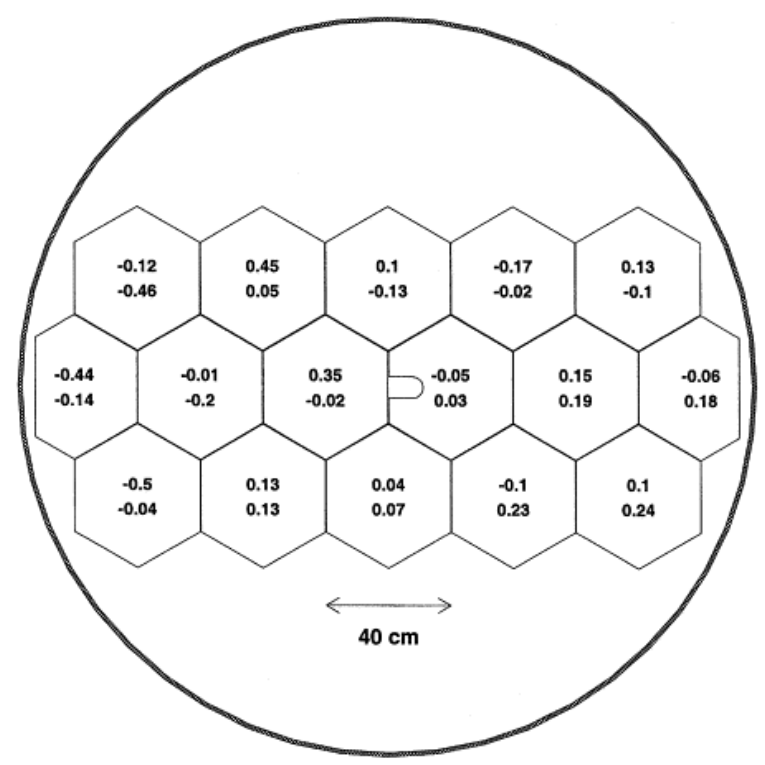

Figure 3.10: Mirror layout in the vessel. The numbers show the deviation from the average center of curvature for the horizontal (top) and vertical (bottom) coordinates in centimeters [25].

and has a reflectivity $>85 \%$ at $160 \mathrm{~nm}$. The average radius of the mirrors is $19.8 \mathrm{~m}$ with a deviation of less than $5 \mathrm{~cm}$ RMS between mirrors as well as on one mirror.

After mounting the mirrors on the honeycomb panel, alignment was performed using a laser, the mirror angles were adjusted until the reflected spot was observed back at the center of curvature.

\section{Photon Detectors}

The phototube holder plate at the focal plane is shown in Figure 3.11. The phototube matrix consists of 2848 ( 89 columns $\times 32$ rows) hexagonally close packed (0.635 in. spacing) holes of approximately 0.6 in. diameter.

One side of each hole is a straight channel 2 in. in depth, which is used to support 

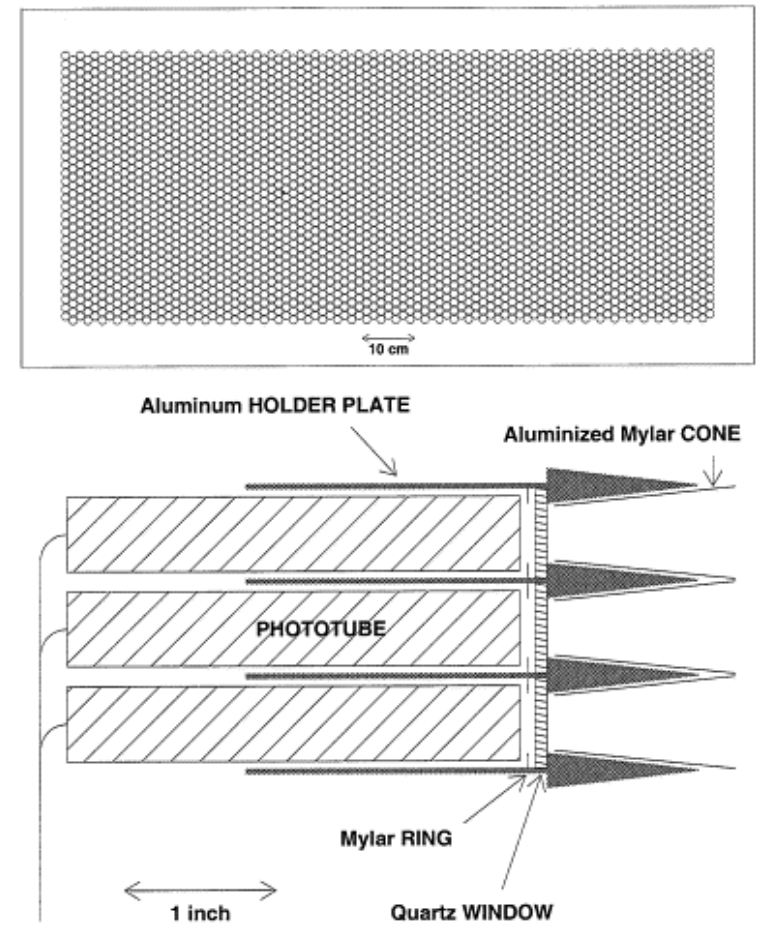

Figure 3.11: Front view (top) and partial cross section (bottom) through the phototube holder plate [25].

a phototube. A quartz window of $2 \mathrm{~mm}$ thickness was inserted into this side of the hole and glued in place. The other side of each hole is a tapered channel 1 in. in depth, with an inner radius of 0.4 in. An aluminized mylar cone is inserted into this side for each phototube and extends slightly out from the block in order to give essentially $100 \%$ coverage for detecting photons.

Two different types of $\frac{1}{2}$ in. diameter photomultipliers are used. The first is a commercially available Hamamatsu tube (R760) which has a quartz window allowing photon detection down to $170 \mathrm{~nm}$. It has a quantum efficiency of approximately $25 \%$ at its peak wavelength of $350 \mathrm{~nm}$. The second is a Russian tube (FEU60) which has a glass entrance window. These tubes were coated with wavelength shifter to reach 
the same wavelength range as the R760 tubes. The FEU60 tubes have an average efficiency of only $36 \%$ compared to the R760 phototubes, i.e. $9 \%$ quantum efficiency. Tubes are grouped by operating point so that each column of 32 tubes can be run at the same high voltage.

In March 2004, due to a short on an FEU base, the RICH PMT array caught on fire and about 600 phototubes were damaged. The remaining tubes were spread throughout the array: in the central part of the phototube holder plate the Hamamatsu R760 tubes were installed in every $4^{\text {th }}$ column starting at column 17 and ending at column $73 ; 15$ columns were equipped with R760 tubes. The empty columns are located in every $4^{\text {th }}$ column between column 0-40 and column 50-89. In the rest of the matrix, FEU60 tubes were installed.

To accommodate the wide range of operating voltages for the phototubes and the large number of tubes, six chains of air-cooled Zener diodes were used, each driven by a high voltage power supply delivering $200 \mathrm{~mA}$. The voltage for each of the active columns was chosen based on the valid range of operating voltages for the set of tubes on the column.

The phototubes are grouped in sets of 16, two sets comprising a column of 32 phototubes in the $89 \times 32$ matrix. The output signals from a group of 16 phototubes are soldered onto paddle cards. The paddle cards plug into a backplane in one of three crates, located on top of the phototube box.

The readout electronics, mounted on cards in the crates, consist of a x20 differ- 
ential amplifier and an analog comparator - one set per PMT, 32 sets per cards, and one card for each PMT column.

The front-end electronics originally used in the SELEX experiment were completely replaced. The electronics prototype was produced at Fermilab and the production boards were fabricated and tested at Harvard University.

The front-end electronics cards are arranged into 3 crates, with 30 cards in each of the first two crates and 29 cards in the third crate. Each crate is controlled by one VME controller board.

\subsubsection{Electromagnetic and Hadronic Calorimeter}

There are two calorimeters at the downstream end of the apparatus, an Electromagnetic Calorimeter (EMCAL) and a Hadronic Calorimeter (HCAL) [34]. The lengths of these are 0.35 and 9.7 interaction lengths respectively. The transverse dimensions are $152.4 \mathrm{~cm}^{2}$ and $100 \mathrm{~cm}^{2}$ respectively. One of the main purposes of the EMCAL is to detect and measure the angles and energies of forward going photons. The HCAL detects neutrons and other hadrons and measures their energies.

Figure 3.12 shows a side view of the EMCAL and HCAL. The EMCAL consists of 5 horizontal and 5 vertical planes of proportional chambers attached to 0.2 inch-thick lead (absorber) sheets. Each layer has 1 radiation length of lead and 8 chambers. The proportional tubes were constructed from aluminum extrusions with anode wires strung through the center of each tube. The anode wires are $162 \mathrm{~cm}$ long and $25 \mu \mathrm{m}$ in 
diameter and the wire-to-wire spacing is $2.54 \mathrm{~cm}$. There are 8 chambers and 64 wires in each plane and horizontal and vertical wire planes are stacked alternately to measure $x$ and $y$ positions. After assembly, the active volume is $162 \mathrm{~cm} \times 162 \mathrm{~cm} \times 31 \mathrm{~cm}$. The EMCAL is 10 radiation lengths.

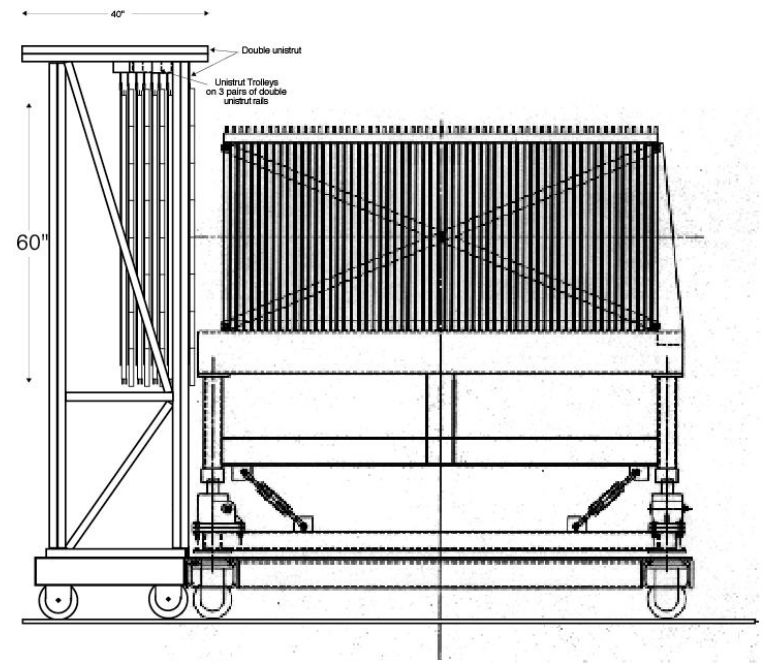

Figure 3.12: Side view sketch of the calorimeters.

The tubes use a gas mixture of $\mathrm{P} 10$ and $\mathrm{CF}_{4}$ in an $86 \%$ - 14\% ratio. The operating voltage for the anode wires is $+2.3 \mathrm{kV}$. The operating voltage was chosen with the intent of maximizing dynamic range while at the same time providing sensitivity for the detection of minimum ionizing radiation.

Pulses from the wires are shaped and amplified after which they are digitized by ADCs and read out through a custom CAMAC module. The entire set of 640 wires is digitized by 4 ADCs in parallel.

The HCAL is situated behind the ECAL, and it is the most downstream detector. 
The calorimeter specifications are given in Table 3.1. The calorimeter is mounted on a stand with jacks that allow limited vertical movement. A more detailed side view of the HCAL is found in Figure 3.13, showing showing fibers, light guides, and photomultipliers. The calorimeter is composed of 64 layers of $24.1 \mathrm{~mm}$ thick Fe and $5 \mathrm{~mm}$ thick scintillator, giving a sampling fraction of $3.5 \%$ and a total thickness of 88.5 radiation lengths and 9.6 interaction lengths. Its active area is $0.990 \mathrm{~m}$ wide by $0.980 \mathrm{~m}$ high.

Table 3.1: Hadronic calorimeter specifications [34].

\begin{aligned} & \hline Type: Sampling (Fe:scintillator, 5:1) \\ & Composition $24.1 \mathrm{~mm}$ Fe, $5.0 \mathrm{~mm}$ PS scintillator \\ & Layer depth: $36.93 \mathrm{~mm} \\ &$ Number of layers: 64 \\ & Size $(\mathrm{x} \times \mathrm{y} \times \mathrm{z}): 0.990 \times 0.980 \times 2.388 \mathrm{~m}^{3} \\ &$ Mass: $12667 \mathrm{~kg} \\ &$ Cell size $:(\mathrm{x} \times \mathrm{y} \times \mathrm{z}) 0.495 \mathrm{~m} \times 0.980 \mathrm{~m} \times 16$ layers \\ & Total cells: $8(2 \mathrm{x} \times 1 \mathrm{y} \times 4 \mathrm{z}) \\ &$ Fiber diameter: $2.0 \mathrm{~mm} \\ &$ Fiber separation: $30.0 \mathrm{~mm} \\ &$ Fibers per cell: $16 \times 16=256 \\ &$ Total fibers: $8 \times 256=2048 \\ &$ Interaction length: $2.40 \lambda_{I}$ per cell \\ & $9.62 \lambda_{I}$ total \\ & Radiation length: $22.1 X_{0}$ per cell \\ & $88.5 X_{0}$ total \\ & Sampling fraction: $3.54 \% \\ &$\hline\end{aligned}

The calorimeter is composed of Kuraray SCSN-81 PS scintillator. Each of the 64 sheets of scintillator has 32 keyhole-shaped channels milled into it, and they are separated by $30 \mathrm{~mm}$. The light of the scintillator sheets is brought out by Bicron 


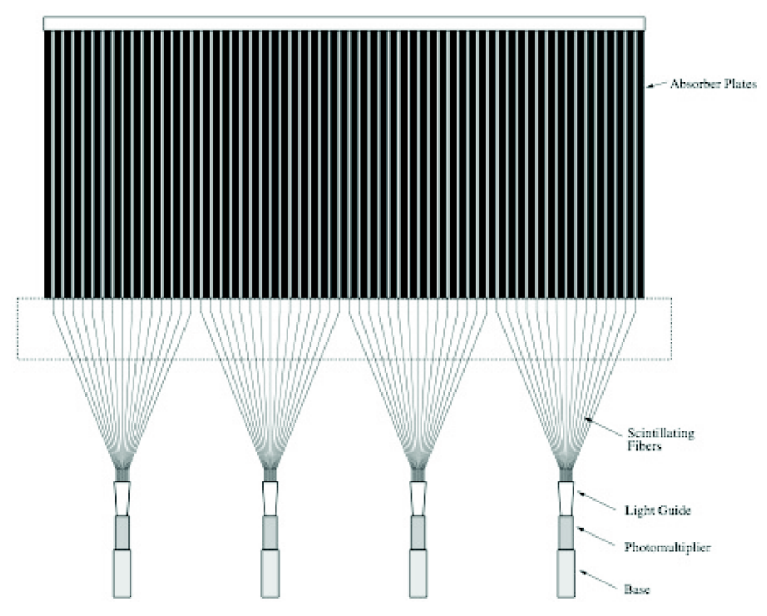

Figure 3.13: Hadronic Calorimeter details.

single-clad BCF-92 wavelength shifting fibers with a $2 \mathrm{~mm}$ diameter.

\subsubsection{Drift and Proportional Chambers}

The MIPP Experiment uses three small drift chambers (BC1, BC2, BC3) [23] to measure the incoming beam particles (e.g. proton from primary beamline) and six large chambers to track particles downstream of the TPC. Four of the six large chambers (DC1, DC2, DC3, DC4) are drift chambers similar to the beam chambers in design. The two chambers in front and behind of the RICH detector are proportional wire chambers (PWC5 and PWC6) [22].

All nine chambers have four planes of wires. The Beam Chambers have 160 wires per plane, and they are all identical. DC1 has 512 wires per plane. DC2, DC3 and DC4 have 512 wires on plane 2 and plane 3, but only 448 channels on plane 1 and plane 4 with wires at larger angles. The two PWCs have 640 wires per plane. The 
total number of wires read out is 14848 .

The 9728 BC/DC channels are read out through 1216 8-channel pre-amps and 304 32-channel discriminators, while the PWCs have 160 32-channel pre-amps. During the run, the BCs and the DCs were read out with LeCroy 4291 TDCs, and the PWCs used RHM (receiver-memory-hybrid) electronics [35].

The wire spacing is $1.016 \mathrm{~mm}$ in the Beam Chambers, $3.4925 \mathrm{~mm}$ in DC1, $3.175 \mathrm{~mm}$ in DC2, DC3 and DC4, and $3.0 \mathrm{~mm}$ in the PWCs. The BCs and DCs have wires at angles of $\pm 7.93^{\circ}$ and $\pm 21.6^{\circ}$ to the vertical. The PWCs measure $x$ in plane 1 (vertical wires), $y$ in plane 2 (horizontal wires), and angles of $\pm 28.07^{\circ}$ in planes 3 and 4 . The active area is $15.24 \times 10.16 \mathrm{~cm}^{2}$ in the BCs, $182.88 \times 121.92 \mathrm{~cm}^{2}$ in DC1, $152.4 \times 101.6 \mathrm{~cm}^{2}$ in DC2, DC3 and DC4, and $200 \times 200 \mathrm{~cm}^{2}$ in the PWCs.

\subsubsection{Magnets}

MIPP employs two the analysis magnets known as Jolly Green Giant (JGG) and ROSIE.

JGG consists of four sets of coils, two sets on top and two below the aperture. Each set has 16 coils inside, and each coil has two turns. All coils in a set are operated in series. Over the course of the MIPP running period, several of these coils failed and were either fixed or removed. As a consequence, there are three field maps. Only the first field map was measured using Zip-tracking field measuring device, and the rest were simulated by computer models for use during reconstruction. In each of 
these configurations, the central field is approximately $7 \mathrm{kG}$ and the field near the pole tips is $8 \mathrm{kG}$.

\subsection{MIPP Acceptances for NuMI Target}

A key feature of the MIPP experiment is its excellent particle identification capabilities. Almost all particle species across the entire kinematic range that can be produced from the $120 \mathrm{GeV} / c$ primary proton beam can be unambiguously identified through a combination of the experiment's sub-detectors. This is illustrated in Figure 3.14. These detectors have nearly $100 \%$ acceptance over the full range of secondary momenta relevant to the MINOS experiment and track-by-track particle identification can be done at roughly the $3 \sigma$ level over the same range. The data set taken by MIPP using the NuMI target should therefore provide a direct input to the simulations of the NuMI horn focusing system essentially eliminating hadron production as a significant source of uncertainty in the absolute prediction of the Near and Far detector spectra.

The overall acceptance regions for tracking pions and kaons in the $\mathrm{RICH}$ are shown in Figure 3.15(a) and Figure 3.15(b) respectively. 


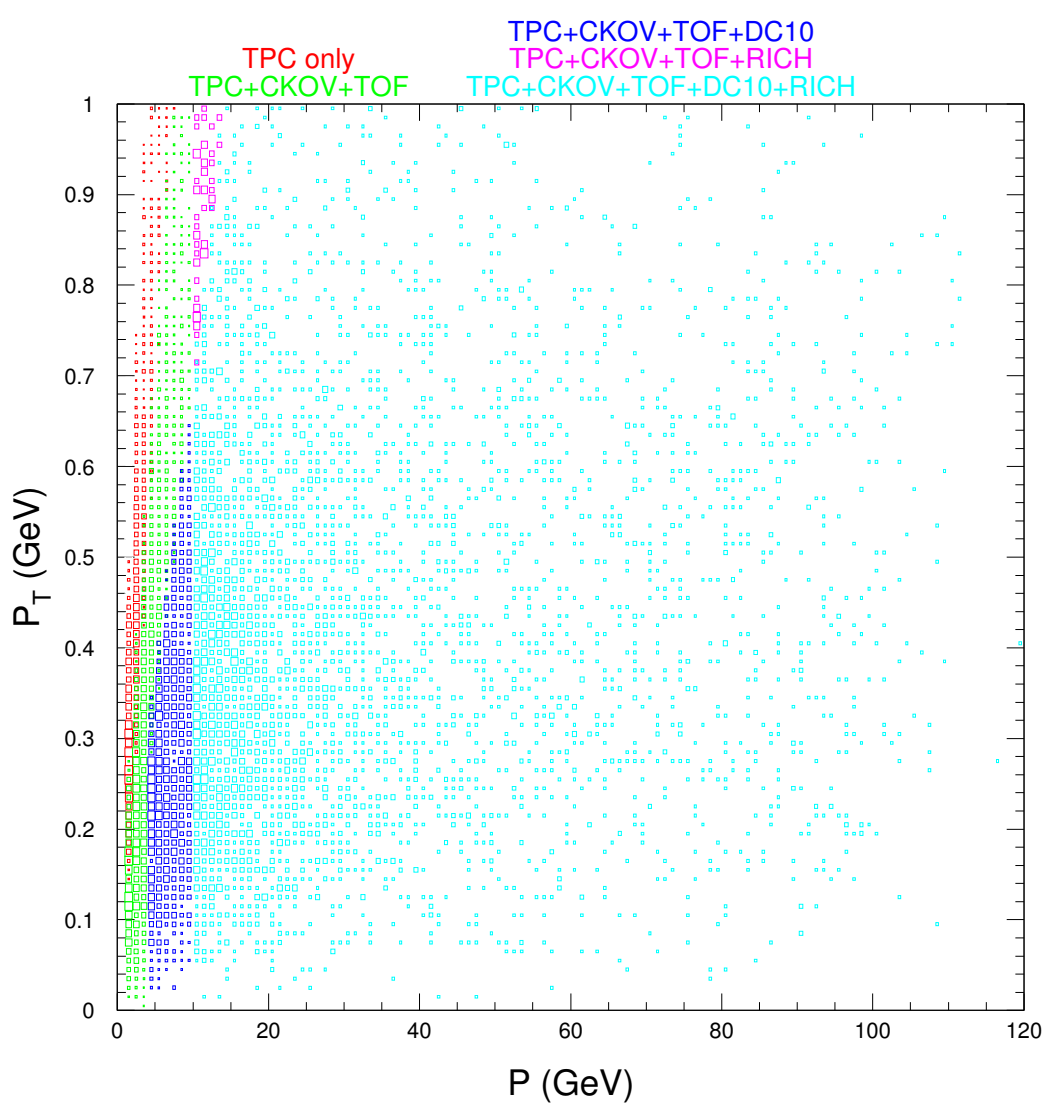

Figure 3.14: The acceptance of the various sub-detectors in the MIPP experiment as a function of secondary particle momentum and transverse momentum.
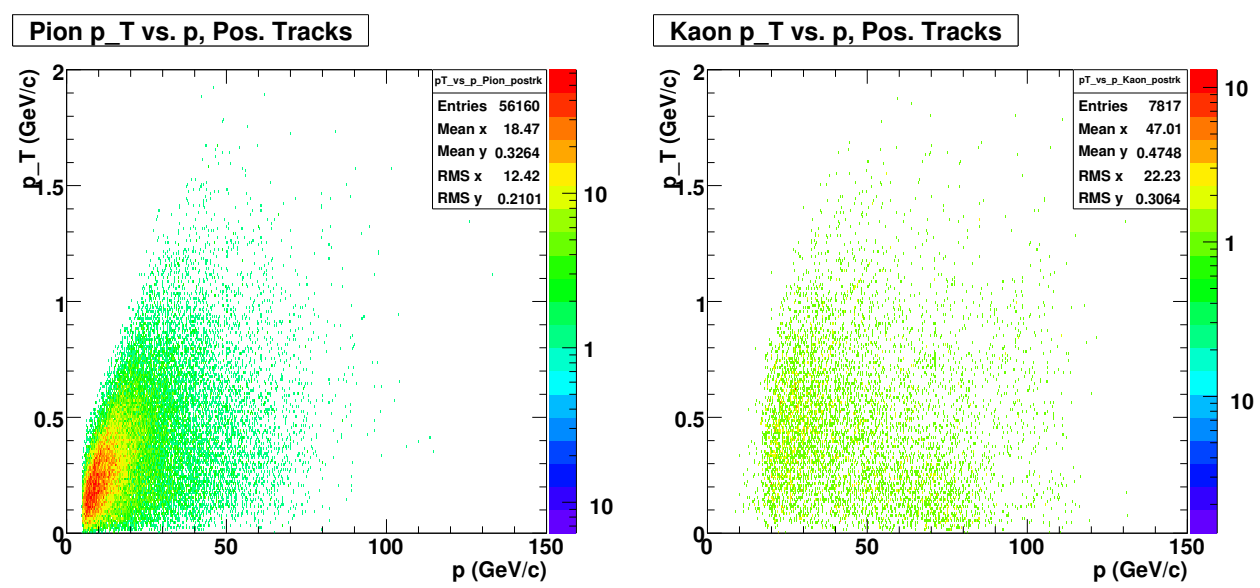

Figure 3.15: $p_{t}$ vs. $p$ spectrum for all positive (a) pion and (b) kaon tracks coming off of the NuMI target. Pions were selected using the RICH particle identification log-likelihoods [described in Subsection 4.3.3]. 


\section{Chapter 4}

\section{Event Reconstruction}

The event reconstruction proceeds through several steps. Firstly, a set of hits in the TPC and chambers is used in conjunction with the magnets to measure and calculate the 3-dimensional spatial coordinates, momentum and charge of charged particles. The complete kinematic description of an event requires that the mass of each particle is specified in addition to its momentum. This is achieved through particle identification using one or more of the sub-detectors. For the NuMI target analysis, the goal is to distinguish pions, kaons and protons. The RICH sub-detector provides this ability above $20 \mathrm{GeV} / c$. Below $20 \mathrm{GeV} / c$, the TPC, the TOF and the differential Cherenkov detector can be used. However, at the time of writing, these had yet to be implemented. For this reason, the main focus of this chapter is particle identification with the RICH and hence for particles above $20 \mathrm{GeV} / c$. 


\subsection{Monte Carlo Simulation}

A Monte Carlo was developed to simulate the MIPP experiment. The MIPP Monte Carlo simulation is based on FLUKA-2006 [11, 12] for secondary particle production and GEANT [10] for tracking along the beam line and through the detector volumes.

The FLUKA beam simulation uses a modified version of the MINOS implementation of the NuMI target. The modifications are

- The incoming proton beam width is adjusted to that expected from the MIPP primary beamline.

- MIPP does not need to cool the target, so water volumes within the cooling tubes and helium volumes around the target are replaced by air volumes.

- Addition of a magnetic field. The NuMI target in the MIPP apparatus is in the fringe field of the JGG. However, as can be seen in Figure 4.1, for the momentum range concerned the particle production is insensitive to the presence of the field.

\subsection{Tracking}

Before tracking begins, a pre-processing step filters out beam pileup events. The remaining events are subject to a pattern recognition algorithm to identify tracks with at least 5 hits in the TPC and chambers. This step includes the formation of 


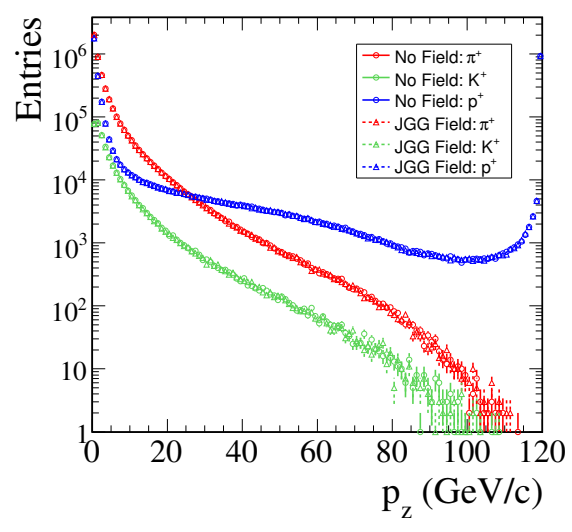

(a) Positive particles.

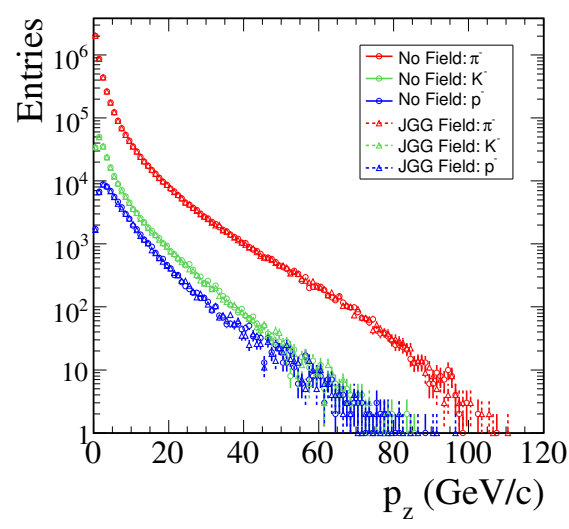

(b) Negative particles.

Figure 4.1: Effect of JGG magnetic field on particle production.

global tracks from track segments in the individual sub-detectors. The resulting global tracks are fitted for momentum based on their curvature in the magnetic fields. A detailed description of the tracking algorithm is presented in Andre Lebedev's thesis [3], a summary of which is given below.

The first major goal of tracking is to combine chamber hits across the length of the experiment into reconstructed tracks. The 9 chambers are grouped into three closely spaced sets $(\mathrm{BC} 1 / 2 / 3, \mathrm{DC} 1 / 2 / 3$ and $\mathrm{DC} 4 / \mathrm{PWC} 5 / 6)$ and each group is used to form a 3-dimensional track segment. The three track segments are then matched in order to form a chamber track candidate which can be fit for momentum based on its curvature in the ROSIE magnetic field.

The reconstruction of a track segment proceeds as follows: starting with hit wire 
clusters in each plane of each chamber, all possible wire crosses between planes are found. These crosses are then examined across all three chambers in a group to find a consistent track segment. If a track segment has an acceptable $\chi^{2}$, the procedure continues.

Chamber track candidates are then found by matching the track segments of DC1/2/3 and DC4/PWC5/6. The candidate is then checked for consistency with a $\mathrm{BC} 1 / 2 / 3$ track segment and matched if possible. Each track segment corresponds to a set of chambers in a field free region. The segments therefore form straight lines which specify the entry and exit points to the ROSIE magnetic field. This allows the momentum and direction of the particle trajectory to be calculated.

The TPC reconstruction is the next major step in the track reconstruction procedure [36]. The algorithm adopted has been adapted from that used by BNL E910 [20]. A global tracking algorithm is used to combine the TPC tracks with the chamber track candidates. Finally, a vertexing algorithm checks whether fully reconstructed tracks originate from a common point. This step is combined with a final vertexconstrained track fit to determine the momentum and direction from all available information.

\subsection{Particle Identification}

In this section, the algorithms used for particle identification with the RICH detector are described. 
Cherenkov light is generated in a carbon dioxide radiator $\left(n-1 \simeq 481 \cdot 10^{-6}\right)$ of length $L=986.827 \mathrm{~cm}$. Figure 4.2 shows a display of one event in the RICH. This display shows the signals observed in the RICH, the particle track vertex extrapolated from the chambers, and the predicted rings for the mass hypotheses $\pi, \mathrm{K}$ and p. Also, the momenta and charges of the tracks are given. Cherenkov photon signals along the predicted rings are clearly seen.

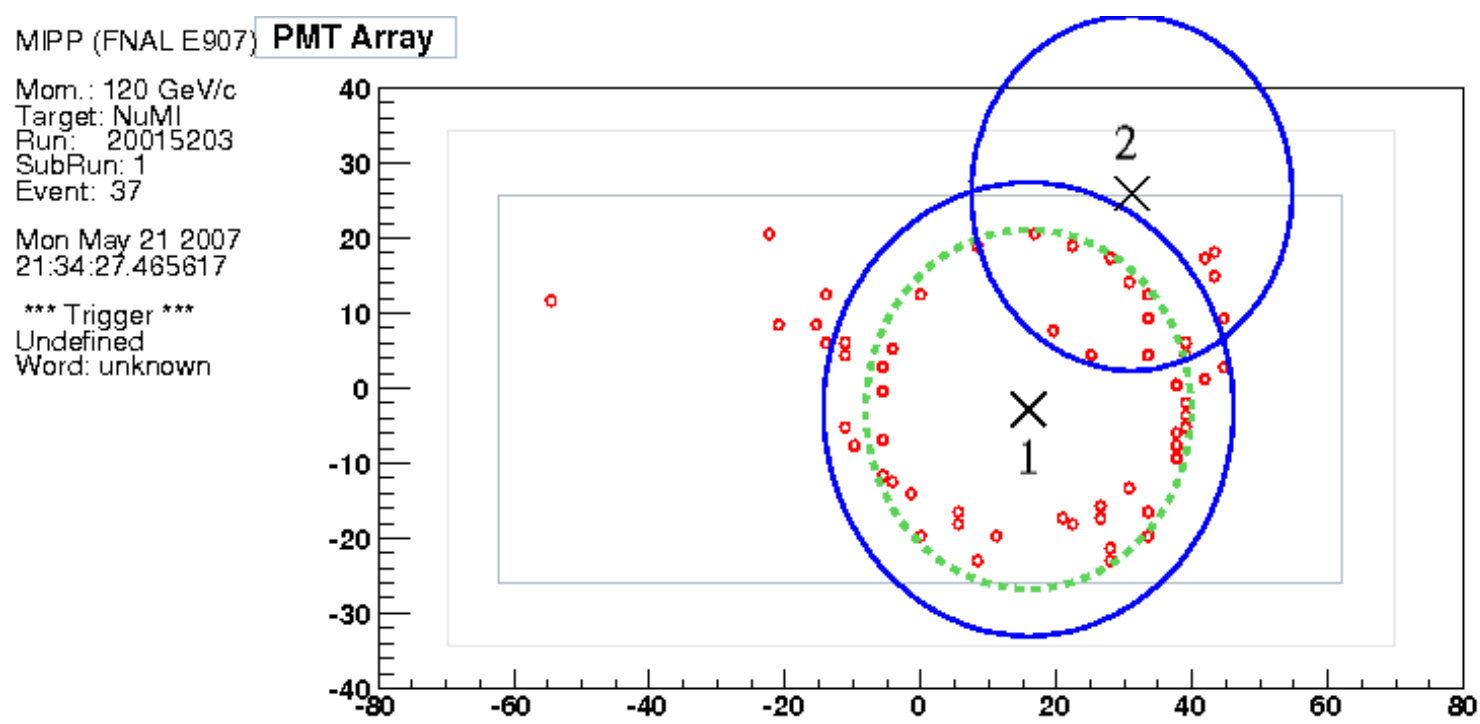

Figure 4.2: Event display in the RICH. Solid lines: rings predicted for the $\pi$ mass hypothesis; dashed line: K hypothesis; crosses: predicted ring center from tracking. Cross 1: $-25.9 \mathrm{GeV} / c$; Cross 2: $-7.2 \mathrm{GeV} / c$.

To interpret the Cherenkov ring information and to construct analysis methods for identifying particles with high efficiency and low misidentification, the expected signals in the detector are now derived. The derivation is based on [37]. 


\subsubsection{The Cherenkov Effect}

The Cherenkov effect occurs when the velocity of a charged particle traversing a dielectric medium exceeds the velocity of light in that medium $(c / n)$, where $n$ is the index of refraction for the medium. Excited atoms in the vicinity of the particle become polarized and coherently emit radiation at a characteristic fixed angle $\theta$, which is determined by the velocity of the particle and the index of refraction of the medium from the relation

$$
\cos \theta=1 / \beta n
$$

with $\beta>1 / n$.

The index of refraction of a material is a function of wavelength, temperature and pressure. Figure 4.3 shows the variation of $n$ with wavelength for $\mathrm{CO}_{2}$, where $n$ decreases with increasing $\lambda$. The variation with temperature is small.

According to Equation 4.1, there is a threshold velocity $\beta_{\text {threshold }}=1 / n$ below which no light is emitted. As the particle velocity increases beyond $\beta_{\text {threshold }}$, the light is given off at larger and larger angles up to a maximum $\theta_{\text {max }}=\cos ^{-1}(1 / n)$ which occurs for $\beta=1$.

The amount of energy emitted per unit length and per unit wavelength interval $d \lambda$ by a singly charge particle is given by [37]

$$
\frac{d E}{d x d \lambda}=\frac{4 \pi^{2} r_{e} m c^{2}}{\lambda^{3}}\left(1-\frac{1}{\beta^{2} n^{2}}\right)
$$

where $r_{e}$ is the classical radius of the electron. The emitted energy is strongly peaked 


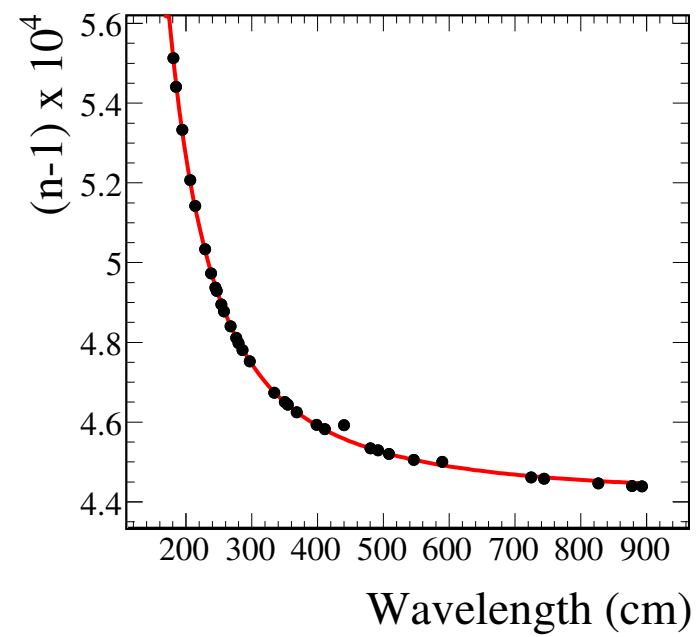

Figure 4.3: Index of refraction of $\mathrm{CO}_{2}$ as a function of wavelength [38].

at short wavelengths. Equation 4.2 can be rewritten in terms of the number $N$ of emitted photons as

$$
\frac{d N}{d x d \lambda}=\frac{2 \pi \alpha}{\lambda^{2}}\left(1-\frac{1}{\beta^{2} n^{2}}\right)
$$

where $\alpha$ is the fine structure constant. The total number of photons emitted per unit path length is

$$
\frac{d N}{d x}=2 \pi \alpha \int_{\beta n>1}\left(1-\frac{1}{\beta^{2} n^{2}(\lambda)}\right) \frac{d \lambda}{\lambda^{2}}
$$

If the variation in $n(\lambda)$ is small over the wavelength region $\lambda_{1}$ to $\lambda_{2}$, the energy emitted per unit length becomes

$$
\frac{d E}{d x}=2 \pi^{2} r_{e} m c^{2} \sin ^{2} \theta\left(\frac{1}{\lambda_{1}^{2}}-\frac{1}{\lambda_{2}^{2}}\right)
$$

while the photon yield is

$$
\frac{d N}{d x}=2 \pi \alpha \sin ^{2} \theta\left(\frac{1}{\lambda_{1}}-\frac{1}{\lambda_{2}}\right)
$$


Using the wavelength interval $155-670 \mathrm{~nm}$, corresponding roughly to the response range of the photomultiplier tubes in the $\mathrm{RICH}$, we find that

$$
\begin{array}{ll}
d E / d x=11200 \sin ^{2} \theta & \mathrm{eV} / \mathrm{cm} \\
d N / d x=2270 \sin ^{2} \theta & \text { photons } / \mathrm{cm}
\end{array}
$$

For a singly charged particle with $\beta \sim 1$ traversing $\mathrm{CO}_{2}(n \sim 1.000481)$, the Cherenkov angle is $1.78^{\circ}$. This implies that $10.8 \mathrm{eV} / \mathrm{cm}$ is given off as Cherenkov radiation, which is small compared to the energy loss due to ionization.

The angular distribution of the light intensity is approximately a $\delta$ function at the Cherenkov angle. The actual distribution observed is broadened due to dispersion, energy loss of the particle, multiple scattering and diffraction in the detector.

\subsubsection{Detector Effect}

The mirrors gather the Cherenkov light produced by a particle traversing the region and focus it onto the PMT array. The reflection coefficient for light at normal incidence on the mirror surface is shown in Figure 4.4. In the visible spectrum the

mirrors have high reflectance. However, in the far ultraviolet region, they have a much lower reflectance.

Another important consideration is the transmission of light through the medium. Carbon dioxide has absorption bands in the far ultraviolet portion of the spectrum. Figure 4.5 shows the region over which $\mathrm{CO}_{2}$ has a high absorption. 


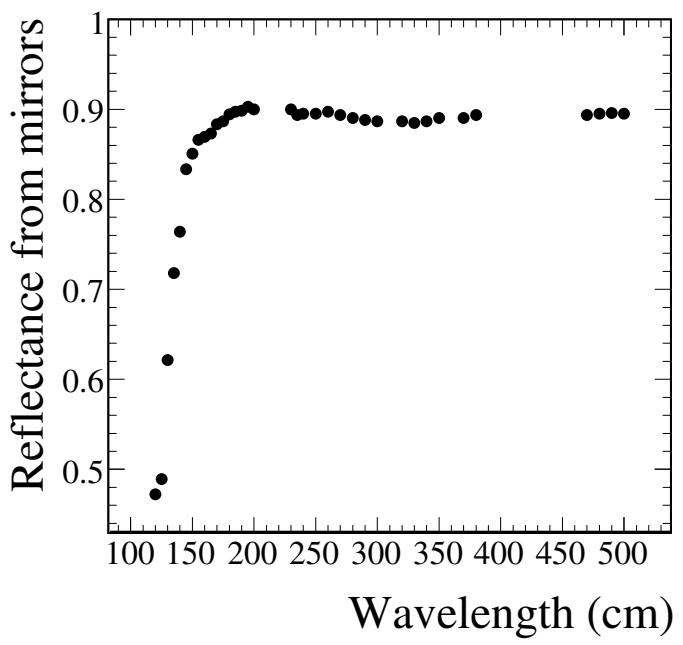

Figure 4.4: The reflectance at normal incidence from mirrors [39].

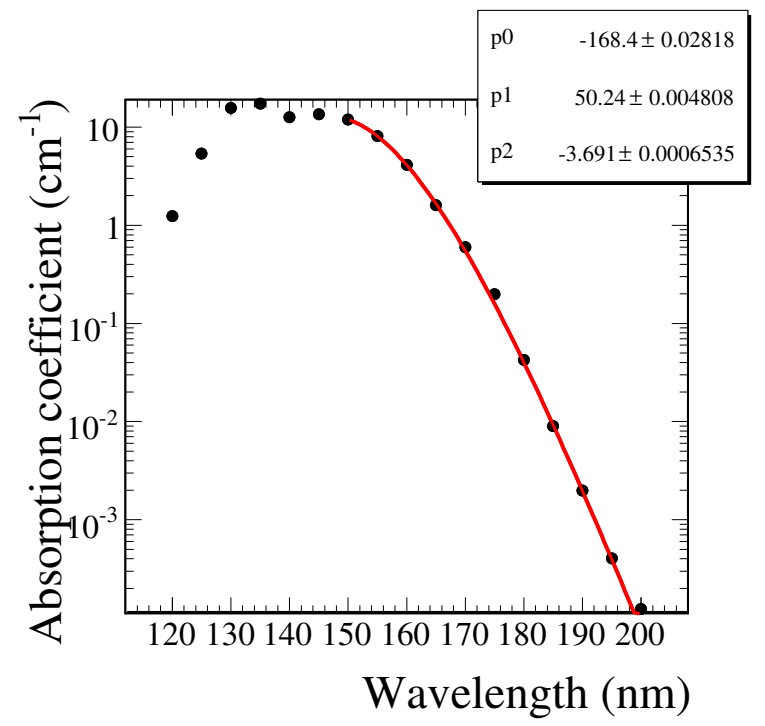

Figure 4.5: Absorption region of Carbon Dioxide [40].

Figure 4.6 shows light transmission efficiency for the PMT and its housing. Figure 4.6(a) is the efficiency of reflection from the cones as a function of wavelength; 
Figure 4.6(b) is the efficiency with which light is transmitted through the quartz window. Note that the quartz allows light transmission below $200 \mathrm{~nm}$; finally, Figure 4.6(c) shows the PMT quantum efficiency as a function of wavelength.

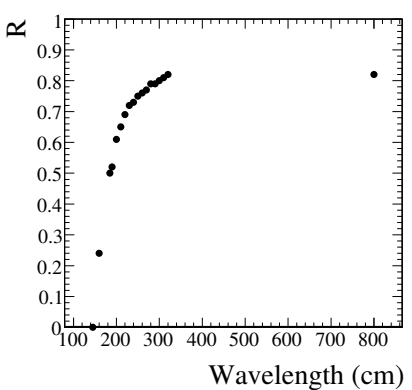

(a) Efficiency of reflection from cone.

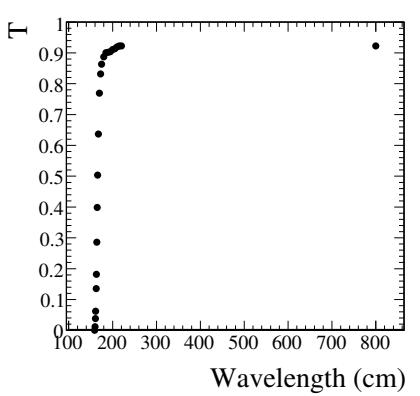

(b) Efficiency of light transmission through ciency. quartz window.

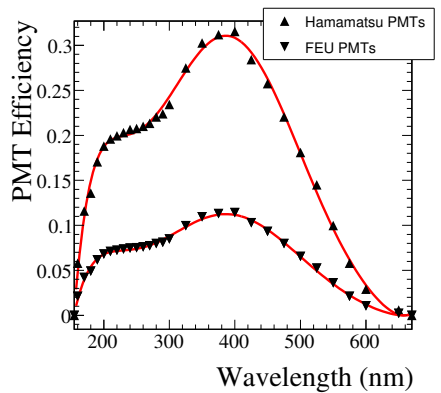

(c) PMT quantum effi-

Figure 4.6: Efficiencies of light transmission of a PMT and its housing as a function of wavelength [39].

The photoelectron output of a given PMT is obtained by convolving the frequency spectrum of produced Cherenkov radiation with the frequency response of the collection system and tube. Thus, using Equation 4.4 for the number of photons produced per unit path, we find that the number of emitted photoelectrons in the tube per unit particle pathlength is

$$
\frac{d N_{e}}{d x}=2 \pi \alpha \int\left(1-\frac{1}{\beta^{2} n^{2}}\right) \epsilon_{c}(\lambda) \frac{d \lambda}{\lambda^{2}}
$$

where $\epsilon_{c}(\lambda)$ is the efficiency for collecting photons of wavelength $\lambda$ at the cathode. 
The number of photoelectrons can then be written in the form

$$
N_{e}=N_{0} L \sin ^{2} \theta
$$

where $L$ is the length of the radiator and the various efficiencies and spectral responses are incorporated in the constant $N_{0}$.

The index of refraction of the gas is related to its density $\rho$ through the LorenzLorentz law [37]

$$
\frac{n^{2}-1}{n^{2}+2} \frac{M}{\rho}=R
$$

where $M$ is the molecular weight and $R$ is the molecular refraction coefficient.

Since for gases $n \simeq 1$, Equation 4.10 can be rewritten to a high degree of accuracy as

$$
n-1 \simeq \frac{3}{2} \frac{R}{M} \rho
$$

From the ideal gas law

$$
P=\rho R^{\prime} T / M
$$

where $P$ is the pressure, $T$ is the absolute temperature, and $R^{\prime}$ the gas constant. From Equation 4.11 and Equation 4.12, we obtain the following equation

$$
n-1=\left(n_{0}-1\right) \rho / \rho_{0}
$$

where the subscript 0 indicates that the quantity is measured at standard temperature and pressure. 


\subsubsection{Maximum Likelihood Method for Particle Identifica- tion}

The RICH exploits the Cherenkov process since only particles whose velocity exceeds some minimum value produce light. As the different particle species have different masses, given a momentum measurement, the RICH can be used to distinguish between particles based on ring radius.

A maximum likelihood approach for particle identification is used. Each particle which crosses the active area of the RICH and which is above the Cherenkov threshold

$$
\gamma_{\text {threshold }}=\frac{1}{1-\beta_{\text {threshold }}^{2}}=32
$$

produces Cherenkov light which is focused by the spherical mirror with focal length, $F=10 m$, onto a ring of radius

$$
R=F \sqrt{\frac{1}{\gamma_{\text {threshold }}^{2}}-\frac{1}{\gamma^{2}}} .
$$

In our case, $R=32 \mathrm{~cm}$ for $\gamma \rightarrow \infty$. The position of the ring center in the focal surface and the ring radius for the different mass hypotheses can be predicted from the parameters of the track and the particle momentum measured in tracking. we only consider the mass hypotheses of $\mathrm{e}, \mu, \pi, \mathrm{K}$ and $\mathrm{p}$.

The method for particle identification is to count the number of observed digits in the "search band": the RICH active region between the smallest physically possible ring radius and the electron radius for a given momentum. The probability of producing each observed digit is calculated for each particle hypothesis. The ring can 
then be associated with the most probable hypothesis. The probability calculation proceeds as follows.

The detection efficiency may be calculated as

$$
\epsilon(\beta)=1-\operatorname{Pr}\left(0, N_{e}\right)
$$

where $\operatorname{Pr}\left(0, N_{e}\right)$ is the probability that no electrons where emitted by the photocathode of the PMT if the average number is $N_{e}$. According to Equation $4.8, N_{e}$ depends on $\beta$, the collection efficiency, and the quantum efficiency of the tube. Since the photoelectron emission follows a Poisson distribution, we have

$$
\epsilon(\beta)=1-\exp \left(-N_{e}\right)
$$

For each mass hypothesis $j$, we assume a probability function to observe photoelectrons $N_{e}^{(i)}$ at each photomultiplier $i$. The probability of observing $N_{e}^{(i)}$ photoelectrons is

$$
P_{j}^{(i)}=f_{j}\left(N_{e}^{(i)}\right) .
$$

The joint probability to find all photoelectrons at their observed positions is the product

$$
\prod_{i=1}^{m} f_{j}\left(N_{e}^{(i)}\right)
$$

where $\mathrm{m}$ is the total number of photomultipliers in the search band.

According to the Poisson distribution in Equation 4.17, if photomultiplier $i$ has a digit, the probability to observe a signal given a mean probability of $N_{e}$ photoelectrons 
is

$$
S_{j}\left(N_{e}^{(i)}\right)=1-\exp \left(-N_{e}^{(i)}\right)
$$

Otherwise, the probability is

$$
S_{j}\left(N_{e}^{(i)}\right)=\exp \left(-N_{e}^{(i)}\right)
$$

We also expect some background hits, which have a constant probability, $B^{(i)}$, of occurring over the surface of each photomultiplier, depending on its type (Russian or Hamamatsu) and not on the hypothesis $j$. This background estimate is calculated from the number of digits seen in the PMT array and is taken from the data set.

If we combine signal $S_{j}\left(N_{e}^{(i)}\right)$ and background $B^{(i)}$, we get the probability for a photomultiplier with detected photoelectrons

$$
f_{j}\left(N_{e}^{(i)}\right)=1-\exp \left(-N_{e}^{(i)}\right)\left(1-B^{(i)}\right)
$$

and the probability for a photomultiplier without a hit

$$
f_{j}\left(N_{e}^{(i)}\right)=\exp \left(-N_{e}^{(i)}\right)\left(1-B^{(i)}\right)
$$

We get the likelihood function for hypothesis $j$

$$
\mathcal{L}_{j}=\prod_{i=1}^{m} f_{j}\left(N_{e}^{(i)}\right)= \begin{cases}\prod_{i=1}^{m}\left(1-\exp \left(-N_{e}^{(i)}\right)\left(1-B^{(i)}\right)\right) & \text { PMT has a hit } \\ \prod_{i=1}^{m} \exp \left(-N_{e}^{(i)}\right)\left(1-B^{(i)}\right) & \text { PMT has no hit }\end{cases}
$$

Thus log-likelihood function reads

$$
\log \mathcal{L}_{j}=\sum_{i=1}^{m} \log f_{j}\left(N_{e}^{(i)}\right)= \begin{cases}\sum_{i=1}^{m} \log \left(1-\exp \left(-N_{e}^{(i)}\right)\left(1-B^{(i)}\right)\right) & \text { PMT has a hit } \\ \sum_{i=1}^{m}\left(\log \left(1-B^{(i)}\right)-N_{e}^{(i)}\right) & \text { PMT has no hit }\end{cases}
$$


If the particle is below threshold, we expect only background (we denote this hypothesis by the index 0 ). In this case, $N_{e}^{(i)}$ is zero and we get the simple expression

$$
\mathcal{L}_{0}= \begin{cases}\prod_{i=1}^{m} B^{(i)} & \text { PMT has a hit } \\ \prod_{i=1}^{m}\left(1-B^{(i)}\right) & \text { PMT has no hit }\end{cases}
$$

To discriminate different mass hypotheses $i$ and $j$, a cut on the log likelihood ratio $R_{i j}=\log \left(\mathcal{L}_{i} / \mathcal{L}_{j}\right)=\log \left(\mathcal{L}_{i}\right)-\log \left(\mathcal{L}_{j}\right)$ is applied. For the analysis of experimental data, simultaneous cuts for different log likelihood ratios, e.g. for $R_{K p}$ and $R_{\pi p}$ are used. These cuts are usually momentum dependent.

This method works well for particle identification in events with only a few rings, but as the number of rings increases, the search bands overlap, and the efficiency goes down and misidentification goes up. Therefore, an extension to the method was developed which favors the information from digits that unambiguously belong to a single ring. This will be described in the following section.

\subsubsection{Iterative Weighting Method}

When analyzing data recorded by the RICH, each digit on the photomultiplier array has to be considered in the evaluation of the likelihood for a given charged track. In cases where ring radii are rather large and track density is high, a considerable overlap of rings appears. In such a case, the peak in the radius distribution corresponding to the digits from the considered track might become obscured by the contribution of digits from the neighboring tracks. In the standard approach, the 
background at the photomultiplier array is measured, and the likelihood functions for various particle hypotheses are calculated as in Subsection 4.3.3.

To improve this method, we notice that most of the background digits actually belong to other tracks in the events, which suggests that an iterative procedure, in which each digit would gradually become predominately associated with one of the tracks, could be used reduce the background level. This method is described in [41], and summarized in this subsection.

For each of the tracks and for each of the photons, the corresponding ring radius is first calculated, and a histogram is filled. In the iterative approach, we proceed to clean up the histograms in the following way: instead of using a weight of one for a given photon in all $n$ histograms, where $n$ is the number of rings, each photon is ascribed a weight such that the sum of weights is equal to one for each photon. The weight for a specific photon in the histogram corresponding to the track $k$ is calculated for the next iteration according to the formula

$$
w_{k}=\frac{y_{k}}{\sum_{k=0}^{n} y_{k}}
$$

where $y_{k}$ is the number of entries in the bin, into which the given photon fits, for histogram $k$. As a result, a photon is given the highest weight in the histogram, where it falls into the peak, and lowest, where it is part of a sparsely populated background.

The likelihood function is then constructed in the following way. Consider each digit $i$ in a given track with the corresponding weight $w_{i}$, the likelihood function 
becomes

$$
\log \mathcal{L}_{j}= \begin{cases}\sum_{i=1}^{m} w_{i} \log \left(1-\exp \left(-N_{e}^{(i)}\right)\left(1-B^{(i)}\right)\right) & \text { PMT has a hit } \\ \sum_{i=1}^{m} w_{i}\left(\log \left(1-B^{(i)}\right)-N_{e}^{(i)}\right) & \text { PMT has no hit }\end{cases}
$$

where the index $i$ runs over all digits in the $e / \mu / \pi / K / p$ hypotheses windows, and number of digits $n_{r}=\sum w_{i}$.

\subsection{RICH Alignment}

The 16 mirrors in the RICH which reflect Cherenkov light onto the PMT array must be aligned in software in order to facilitate ring/track matching. The mirrors are assumed to be transversely shifted from their nominal positions only; longitudinal shifts and rotations are not accounted for in the following procedure. Alignment constants are calculated to be the average shifts based on all runs used in the data sample.

The events are reconstructed as usual and a mirror is determined for each track based on its trajectory. The predicted spatial coordinates of an associated ring center on the PMT array are then calculated. An independent algorithm is also run on the RICH digit information only in order to find and fit the rings. An alignment constant can then be found for each mirror based on the agreement between the track coordinate prediction and that found by the ring-finding algorithm. Figure 4.7 shows the distributions used to determine the constants for each mirror. 

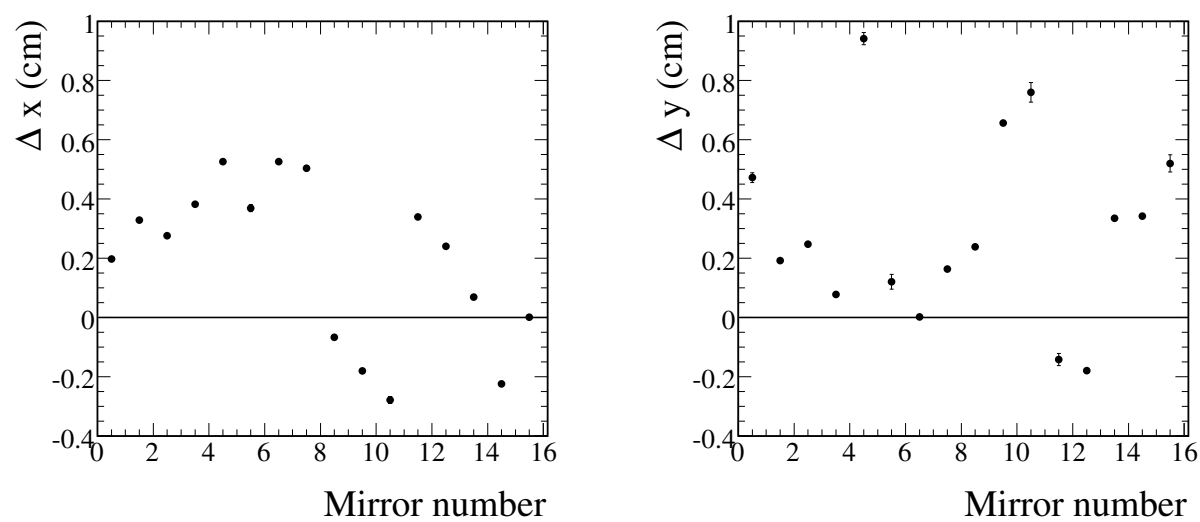

Figure 4.7: RICH alignment constants in $\mathrm{x}$ (left) and y (right) versus mirror numbers.

\subsection{RICH Calibration}

In solving Equation 4.8 for $N_{e}$, an integral over $\lambda$ is required. Light scattering in the detector medium introduces a width to the intrinsic Cherenkov angle which causes the ring radius to be broader and therefore to illuminate a larger region of the PMT array. This broadening will affect the number of photoelectrons per hit PMT and so is partially degenerate with the PMT efficiencies. Also, as shown in Equation 4.13, the index of refraction parameter (n-1) changes proportional to the density of $\mathrm{CO}_{2}$ in the RICH. This parameter is also included in the calibration.

In order to calibrate the amount of Cherenkov light observed at a PMT, four parameters need to be tuned to the data: a width related to light scattering, the Hamamatsu and Russian PMT efficiencies and the index of refraction parameter, (n1). The tuning is achieved by comparing the data and the predicted occupancy of hit PMTs as a function of distance from the ring center; occupancy is defined as the 
number of hit PMTs as a fraction of the total number of PMTs at a particular radius. Occupancy is therefore strongly dependent on light level.

The light scattering width, the PMT efficiency and the (n-1) calibration constants were determined using beam particles from the data with known momenta: the $30 \mathrm{GeV} / c, 60 \mathrm{GeV} / c$ and $120 \mathrm{GeV} / c \mathrm{p} / \mathrm{K} / \pi$ beam. The parameters were adjusted in order to minimize the deviations between the data and the predicted occupancies. Figure 4.8 and Figure 4.9 show the result of this tuning for several beam settings. It can be seen that the prediction closely matches the data. The constants are used when calculating the expected number of photoelectrons in the log-likelihood method. 


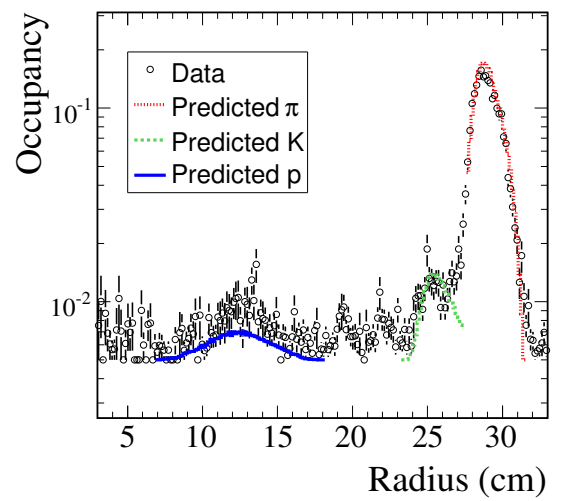

(a) $30 \mathrm{GeV}$ beam

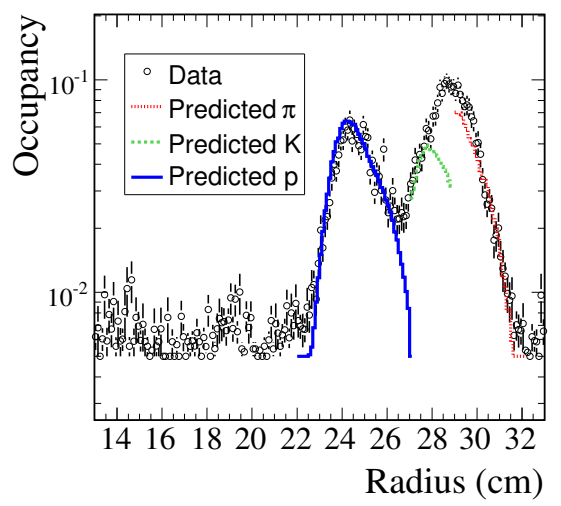

(c) $60 \mathrm{GeV}$ beam

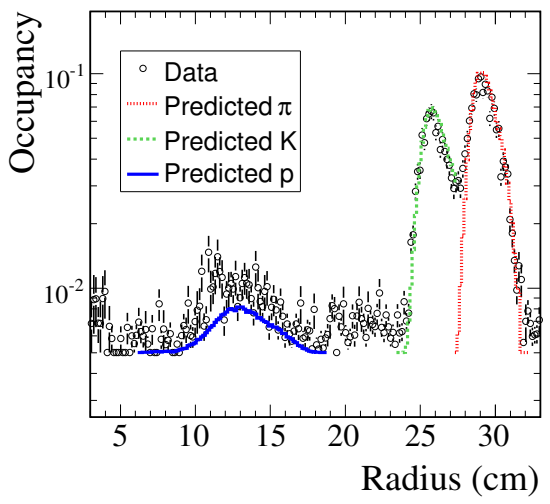

(b) $30 \mathrm{GeV}$ beam

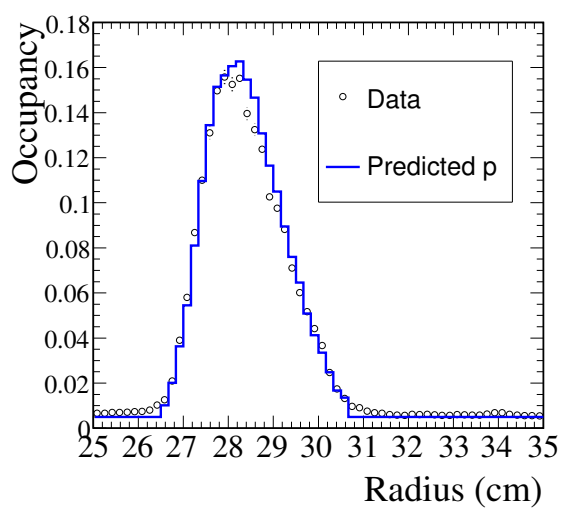

(d) $120 \mathrm{GeV}$ beam

Figure 4.8: The RICH calibration for Russian PMTs. 


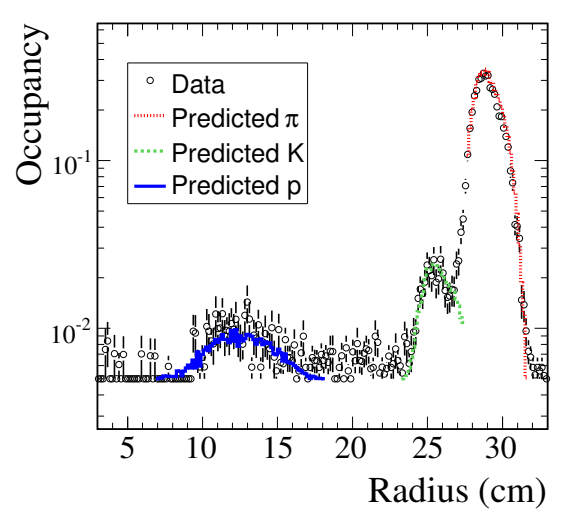

(a) $30 \mathrm{GeV}$ beam

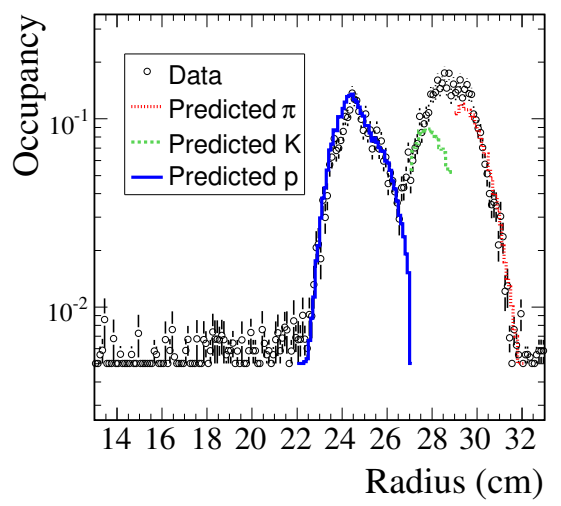

(c) $60 \mathrm{GeV}$ beam

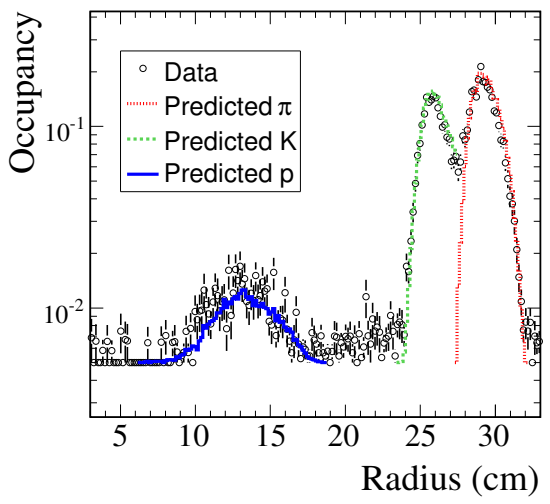

(b) $30 \mathrm{GeV}$ beam

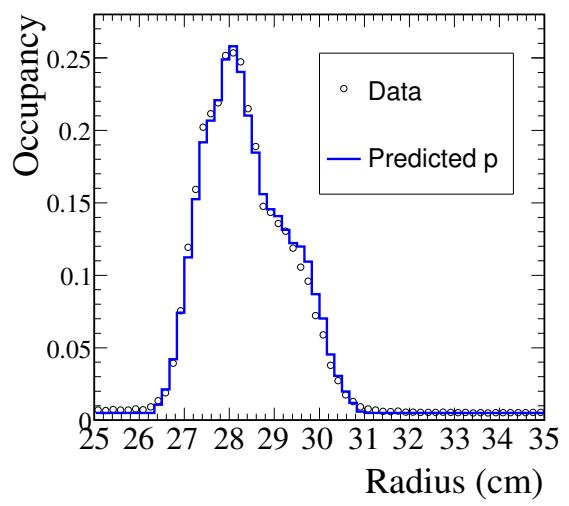

(d) $120 \mathrm{GeV}$ beam

Figure 4.9: The RICH calibration for Hamamatsu PMTs. 


\section{Chapter 5}

\section{NuMI Target Analysis}

This chapter describes the analysis of the NuMI target data. The goal is to measure the production ratios: $\pi^{-} / \pi^{+}, K^{-} / K^{+}, K^{+} / \pi^{+}$and $K^{-} / \pi^{-}$above $20 \mathrm{GeV} / c$ using only the tracking and RICH particle identification capabilities of the MIPP experiment. The analysis strategy and cuts employed are described and the systematic errors resulting from the approach are determined. The final results are then shown in Chapter 6.

\subsection{Event Sample and Cuts}

The experimental setup and the data processing procedure specific to the NuMI data analysis are described in this section. A number of cuts are applied to the data to obtain a clean sample of events. These include the beam definition, the NuMI trigger scheme, track and momentum selection, and particle classification. 


\subsubsection{NuMI Trigger Scheme and Beam Selection}

Two event selection steps are introduced in order to clean up the event sample and to reduce the backgrounds due to the beam.

Firstly, there is a scintillation counter upstream of the NuMI target, consisting of three overlapping scintillators: solid $\left(v_{b}\right)$, with a $2 \mathrm{~mm}$ diameter hole $\left(v_{2}\right)$ and with a $6 \mathrm{~mm}$ diameter hole in the center $\left(v_{6}\right)$, as shown in Figure 5.1. A $2 \mathrm{~mm}$ trigger is defined as $v_{b} \cap \overline{v_{2}} \cap \overline{v_{6}}$, while a $6 \mathrm{~mm}$ trigger is $v_{b} \cap v_{2} \cap \overline{v_{6}}$. The $6 \mathrm{~mm}$ trigger was prescaled by approximately a factor of 10 using hardware logic to resemble the NuMI beam in MINOS, and therefore all data with $2 \mathrm{~mm}$ and $6 \mathrm{~mm}$ triggers have been used without imposing any selection (Figure 5.2). Note that the proton beam spot size obtained from the trigger cuts in data is slightly different from the NuMI Monte Carlo simulations, (which were tuned to reflect the MINOS beam condition). This leads to a small systematic effect which will be described in Subsection 5.3.1.

Secondly, cuts on the beam position are performed. The position of the incoming beam particle is registered in three Beam Chambers. A small fraction of beam particles in the tail of the beam profile beyond $|x|>0.45 \mathrm{~cm}$ and $|y|>0.5 \mathrm{~cm}$ with respect to the beam center are cut. This corresponds to $<0.5 \%$ of the events.

\subsubsection{Track Selection}

The reconstructed interaction vertex is calculated for groups of tracks which are deemed to come from the same interaction. To consider an interaction vertex, it 


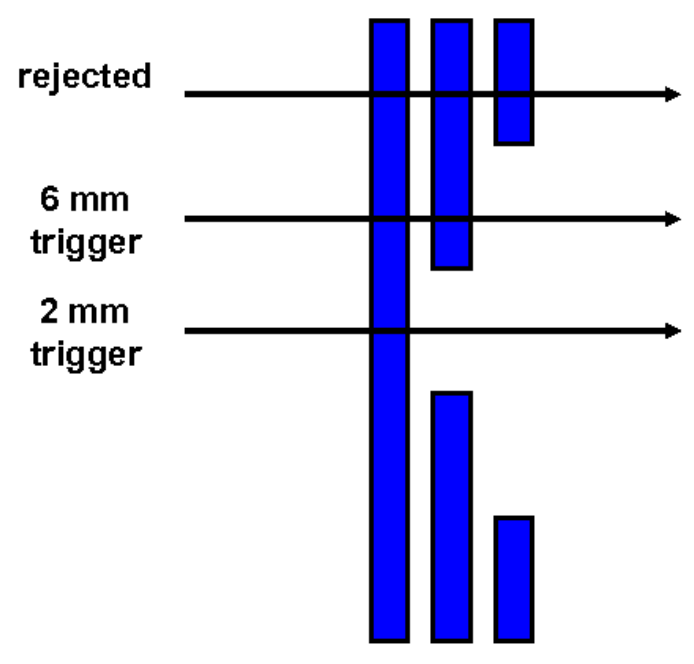

Figure 5.1: A schematic of the NuMI triggers.
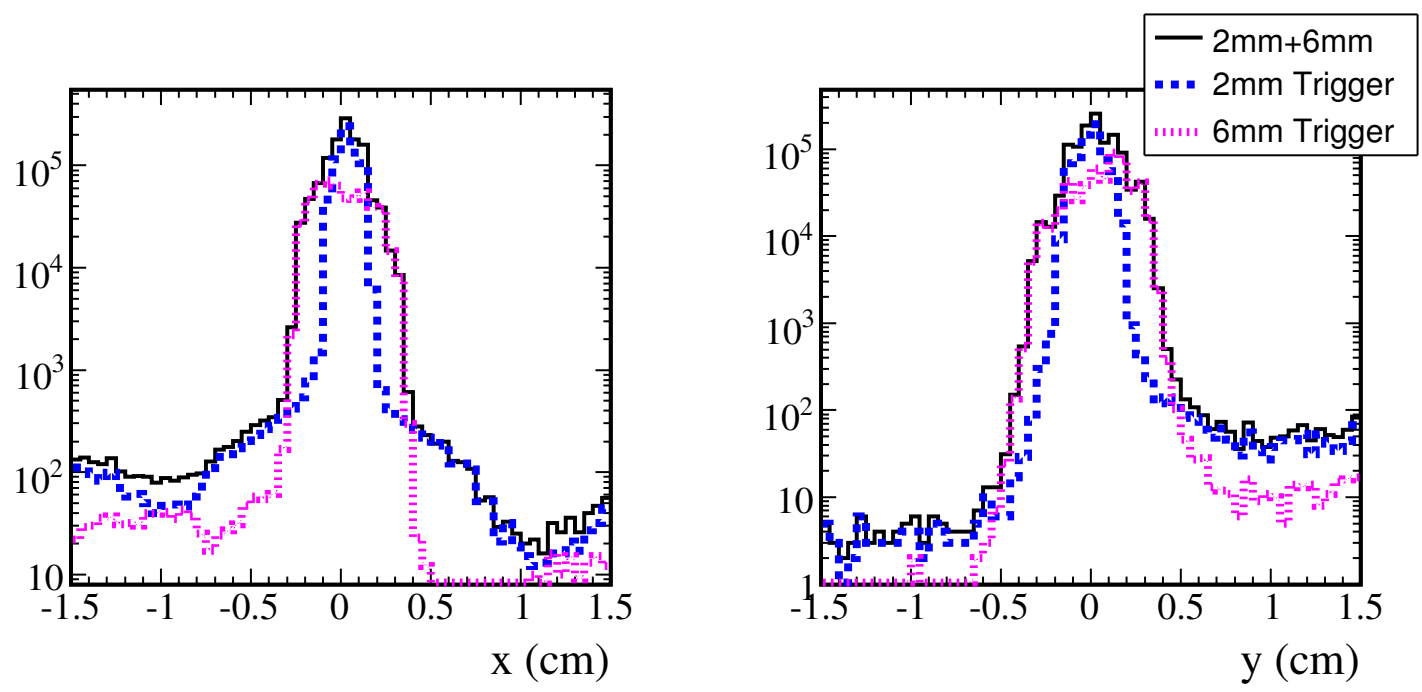

Figure 5.2: Beam positions of $2 \mathrm{~mm}$ and $6 \mathrm{~mm}$ NuMI triggers. 

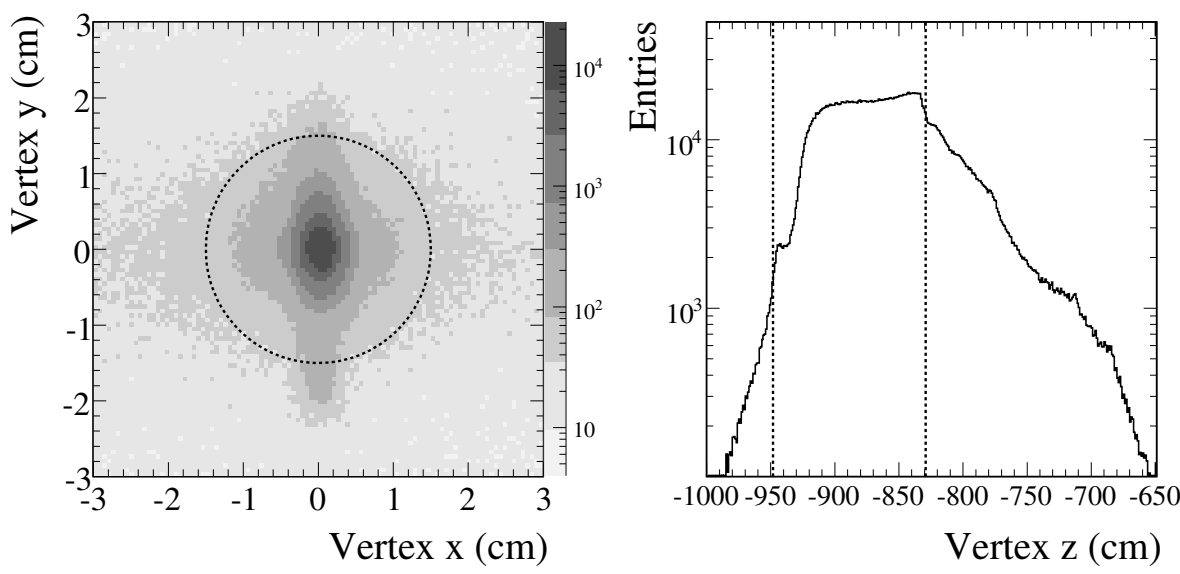

Figure 5.3: Radial and longitudinal reconstructed vertex positions for interaction vertices with $\geq 2$ tracks in data. Dashed lines: cuts on vertices.

must have at least 2 tracks associated with it since vertices with only a single track typically correspond to a non-interacting proton.

Cuts are placed on the interaction vertex position to ensure that the interaction occurred within the target. Figure 5.3 shows the radial, $r$, and longitudinal, $z$, coordinates of the reconstructed vertices in data. Note that the front of the graphite target is at $-928.946 \mathrm{~cm}$ and the back of the graphite target is at $-831.186 \mathrm{~cm}$ in the coordinate system shown in the figure. The vertex is constrained to be in a region around the target by applying the following cuts on $r$ and $z$ :

- Radial: $r<1.5 \mathrm{~cm}$, where $r$ is relative to the axial center of the target

- Longitudinal: -948.266 cm $<z<-829.196 \mathrm{~cm}$

Note that the target region is defined to be the Aluminum cylinder surrounding the NuMI graphite target discussed in Section 3.2 to resemble the am in MINOS. 


\subsubsection{Momentum Selection and Binning}

Due to limitations imposed by the detector acceptance and by the particle identification limit of the $\mathrm{RICH}$, only reconstructed particles with $20 \mathrm{GeV} / c<p_{z}<$ $90 \mathrm{GeV} / c$ and $p_{T}<2 \mathrm{GeV} / c$ are considered. The resulting analysis phase space and momentum binning scheme is presented in Table 5.1.

Table 5.1: Binning scheme in $p_{z}$ and $p_{T}$.

\begin{tabular}{c|r|r}
\hline \hline Bin & $p_{z}(\mathrm{GeV} / \mathrm{c})$ & $p_{T}(\mathrm{GeV} / \mathrm{c})$ \\
\hline$(0,0)$ & $20-24$ & $0-0.2$ \\
$(0,1)$ & $20-24$ & $0.2-0.4$ \\
$(0,2)$ & $20-24$ & $0.4-0.6$ \\
$(0,3)$ & $20-24$ & $0.6-1.0$ \\
\hline$(1,0)$ & $24-31$ & $0-0.2$ \\
$(1,1)$ & $24-31$ & $0.2-0.4$ \\
$(1,2)$ & $24-31$ & $0.4-0.6$ \\
$(1,3)$ & $24-31$ & $0.6-1.0$ \\
$(1,4)$ & $24-31$ & $1.0-1.2$ \\
\hline$(2,0)$ & $31-42$ & $0-0.2$ \\
$(2,1)$ & $31-42$ & $0.2-0.4$ \\
$(2,2)$ & $31-42$ & $0.4-0.6$ \\
$(2,3)$ & $31-42$ & $0.6-1.0$ \\
$(2,4)$ & $31-42$ & $1.0-1.55$ \\
\hline$(3,0)$ & $42-60$ & $0-0.2$ \\
$(3,1)$ & $42-60$ & $0.2-0.4$ \\
$(3,2)$ & $42-60$ & $0.4-0.6$ \\
$(3,3)$ & $42-60$ & $0.6-1.0$ \\
$(3,4)$ & $42-60$ & $1.0-2.0$ \\
\hline$(4,0)$ & $60-90$ & $0-0.2$ \\
$(4,1)$ & $60-90$ & $0.2-0.4$ \\
$(4,2)$ & $60-90$ & $0.4-0.6$ \\
$(4,3)$ & $60-90$ & $0.6-1.0$ \\
$(4,4)$ & $60-90$ & $1.0-2.0$ \\
\hline \hline
\end{tabular}




\subsubsection{Particle Classification}

The likelihood approach for identifying $\pi, \mathrm{K}$ and $\mathrm{p}$ was discussed in Subsection 4.3.3. In each case a track will be identified as the particle with largest likelihood. In regions where the predicted ring radii for $\pi, \mathrm{K}$ and $\mathrm{p}$ are very similar (e.g. at higher momenta), the log likelihood becomes a less effective discriminator for distinguishing the different species. As a result, additional cuts have been developed to improve the selection purity.

Figure 5.4 shows the log-likelihood of $\pi$ and $K$ with respect to the log-likelihood of $\mathrm{p}$ for NuMI Monte Carlo events. The following log likelihood ratio cuts are used for particle classification in the data analysis:

- $R_{\pi^{+} p^{+}}>10$, select $\pi^{+}$

- $R_{K^{+} p^{+}}>35$, select $K^{+}$

- $R_{K^{-} p^{-}}>15$, select $K^{-}$

These numbers were tuned to give the best compromise between high efficiency and high purity (i.e. large background subtraction) [described below in Subsections 5.2.1, 5.2.3 and Appendix A]. For simplicity, these cuts are momentum-independent.

To study particle identification under real experimental conditions, the log-likelihood of data and the Monte Carlo simulation are compared. These comparisons, shown in Figures 5.5, 5.6, 5.7 and 5.8, have been obtained by applying cuts on beam, trigger, position of the vertex. The RICH particle log likelihood ratio cuts listed above were 

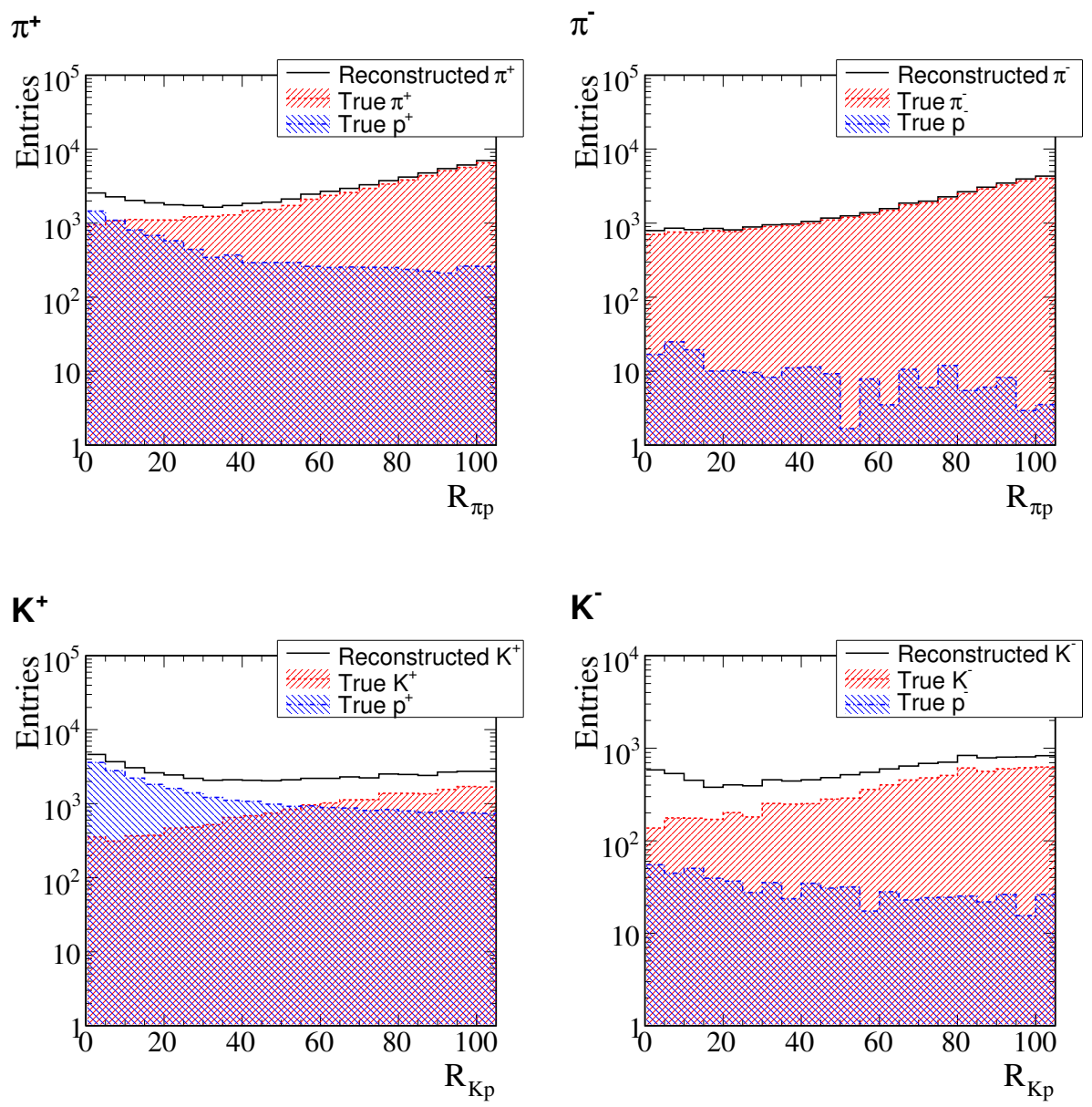

Figure 5.4: Log likelihood ratios for $\pi^{+}$(upper left), $\pi^{-}$(upper right), $K^{+}$(lower left) and $K^{-}$(lower right) with respect to $\mathrm{p}$ in Monte Carlo. Solid lines: reconstructed particle; hatched: true particles. 
also used. The log-likelihood ratio of the RICH particle identification and the next largest log-likelihood is indicated in Figures 5.5, 5.6, 5.7 and 5.8. The distributions are normalized according to the maximum bins in the data. The shape agreement between data and Monte Carlo is reasonably good, although the data distributions are slightly broader than the Monte Carlo.

\subsection{Evaluation of Corrections}

In order to calculate production ratios, it is necessary to first extract true momentum distributions for each particle species. To do this, a series of corrections must be applied to the reconstructed data. There are several reasons for this:

- 10\% of secondary particles interact upstream of the RICH

- $\sim 10 \%$ of particles that pass through the RICH flange (rather than the RICH front window) interact

- $\sim 10 \%$ of kaons decay in flight upstream of the RICH

- Misidentification caused by the likelihood selection method

- Multiple scattering of particles upstream of the RICH leading to underestimated momenta, which in turn leads to misidentification by the likelihood selection method 


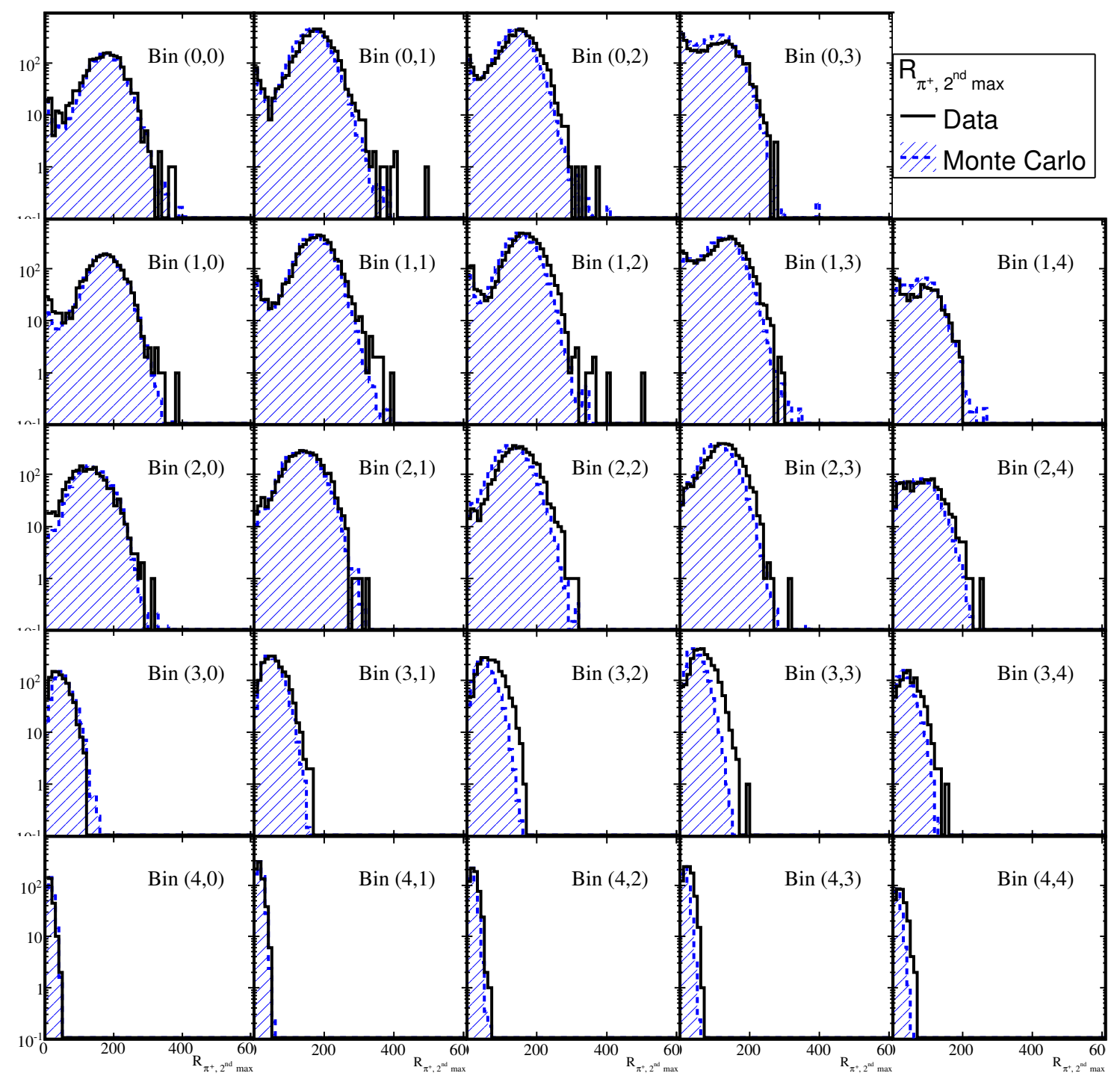

Figure 5.5: Log-likelihood ratio for reconstructed $\pi^{+}$in different momentum bins. The log-likelihood ratio of reconstructed $\pi^{+}$and the $2^{\text {nd }}$ largest likelihood is shown. Solid lines: data; hatched: Monte Carlo simulation. 


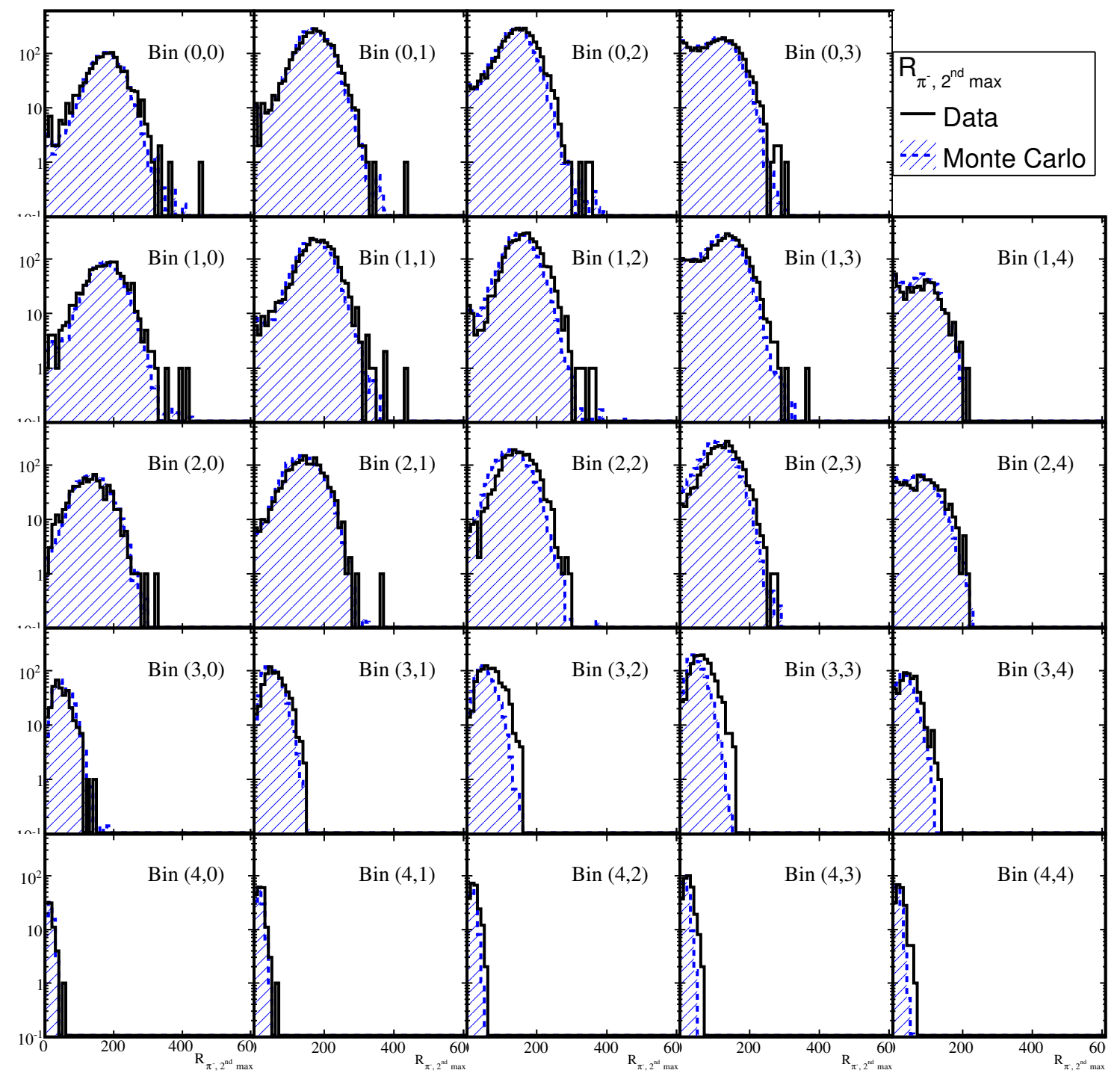

Figure 5.6: Log-likelihood ratio for reconstructed $\pi^{-}$in different momentum bins. The log-likelihood ratio of reconstructed $\pi^{-}$and the $2^{\text {nd }}$ largest likelihood is shown. Solid lines: data; hatched: Monte Carlo simulation. 


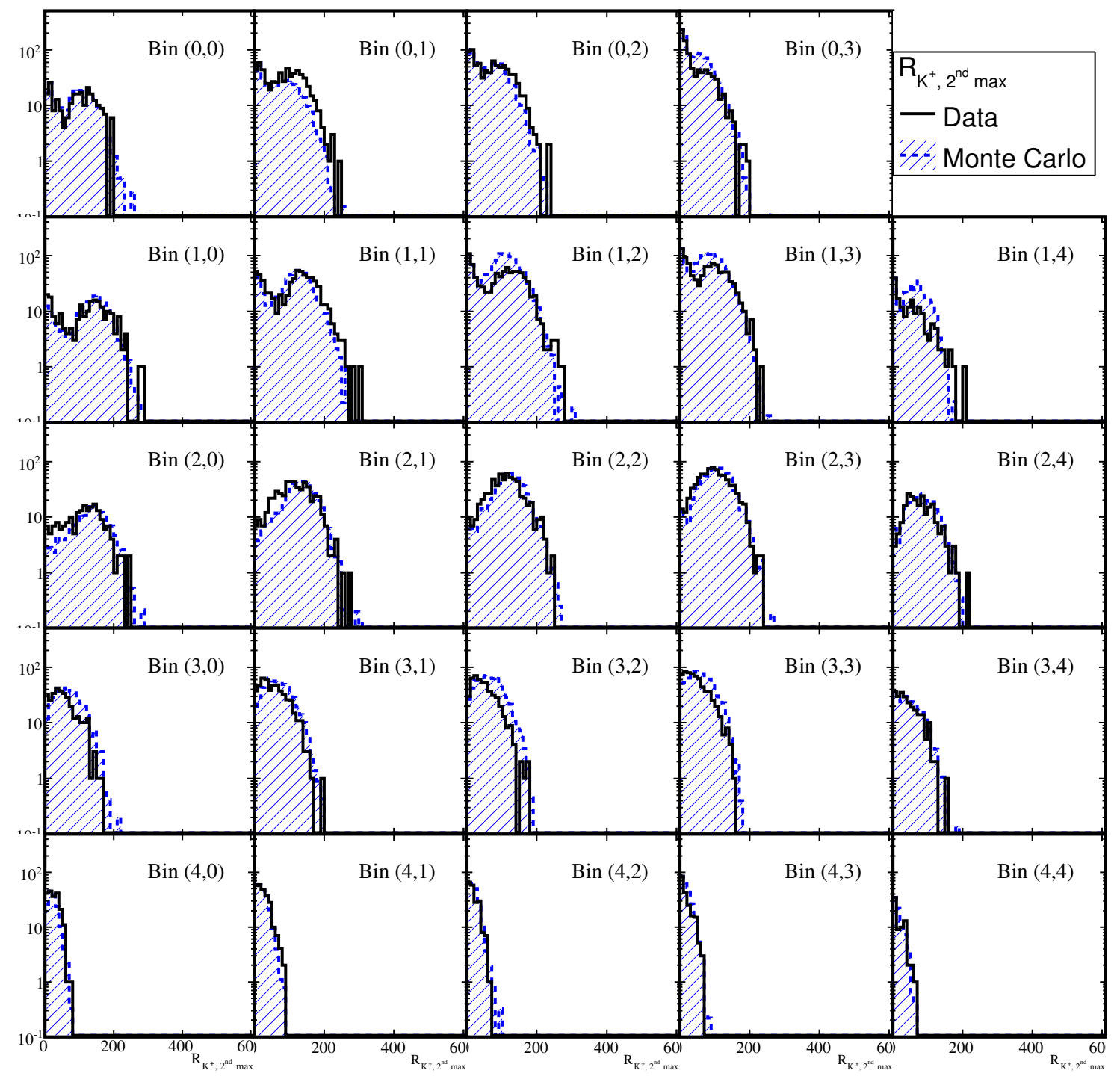

Figure 5.7: Log-likelihood ratio for reconstructed $K^{+}$in different momentum bins. The log-likelihood ratio of reconstructed $K^{+}$and the $2^{\text {nd }}$ largest likelihood is shown. Solid lines: data; hatched: Monte Carlo simulation. 


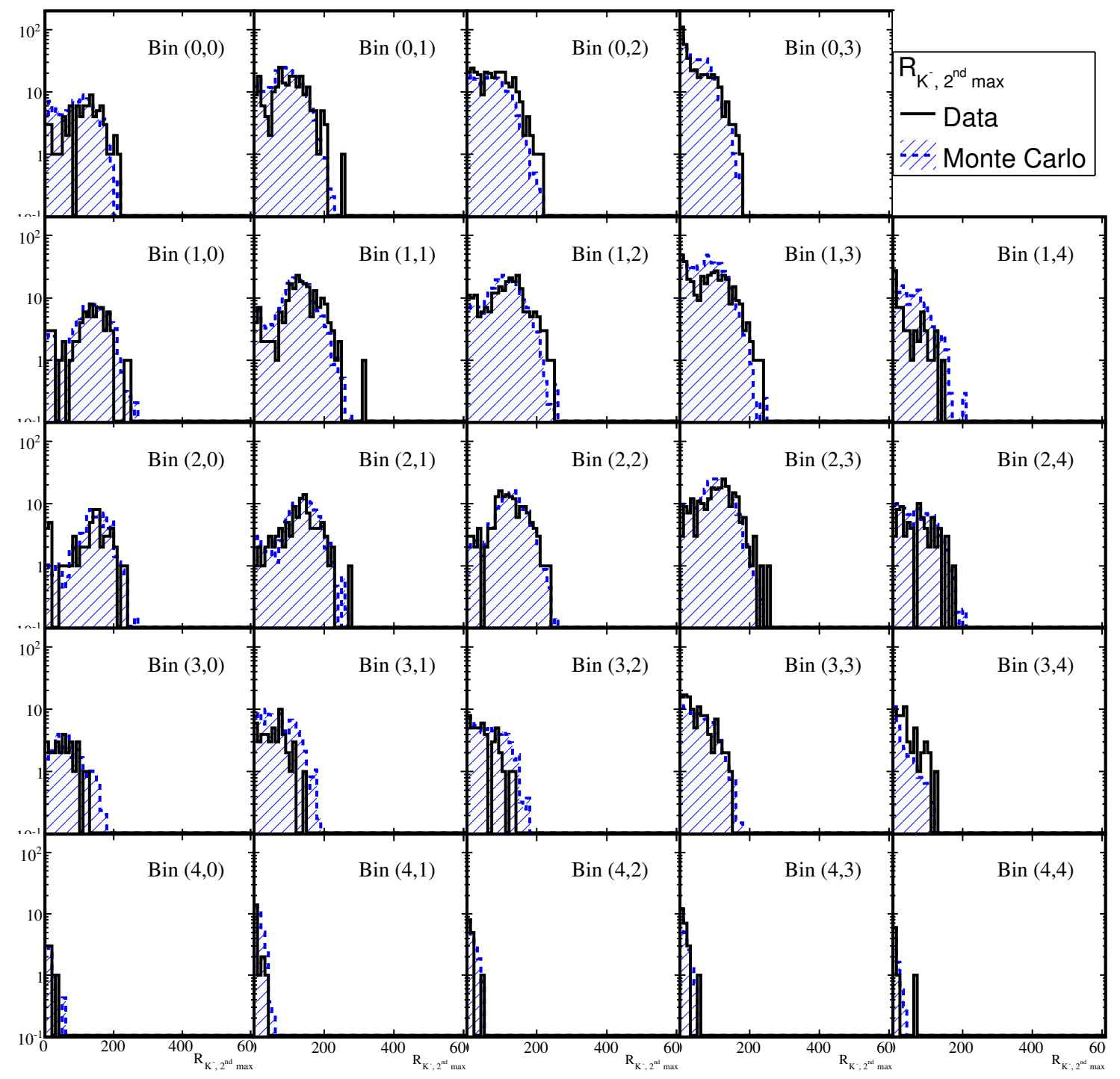

Figure 5.8: Log-likelihood ratio for reconstructed $K^{-}$in different momentum bins. The log-likelihood ratio of reconstructed $K^{-}$and the $2^{\text {nd }}$ largest likelihood is shown. Solid lines: data; hatched: Monte Carlo simulation. 
The Monte Carlo simulation provides a way of correcting for these effects to first order. The corrections calculated for this analysis are

1. Purity correction: subtracts background events from the selected sample.

2. Momentum correction: translates a reconstructed momentum distribution to true momentum.

3. Efficiency correction: accounts for events which are not correctly reconstructed by the algorithms.

The determination of particle identification and its derivation was discussed in Chapter 4. The cuts mentioned in Section 5.1 are applied to the sample before evaluating the correction parameters. These corrections will be described and quantified below.

\subsubsection{Purity Correction}

The first correction applied to data accounts for background contamination in the selected sample. The correction employs the purity as calculated from the Monte Carlo simulation and is defined as

$$
\mathcal{P}_{x}^{i}=\frac{t_{x}^{x}}{t_{x}}
$$

where $t_{x}$ is the number of reconstructed $x, t_{x}^{x}$ is the number of true $x$ reconstructed to be $x, x=\pi^{ \pm}, K^{ \pm}, p^{ \pm}$, and $i$ denotes the reconstructed momentum bin. Figure 5.9 shows the purity versus momentum bin. The $\mathcal{P}_{\pi^{ \pm}}^{i}, \mathcal{P}_{K^{ \pm}}^{i}$ and $\mathcal{P}_{p^{ \pm}}^{i}$ values themselves are the corrections applied bin-by-bin to the data. 
$\pi^{+}$

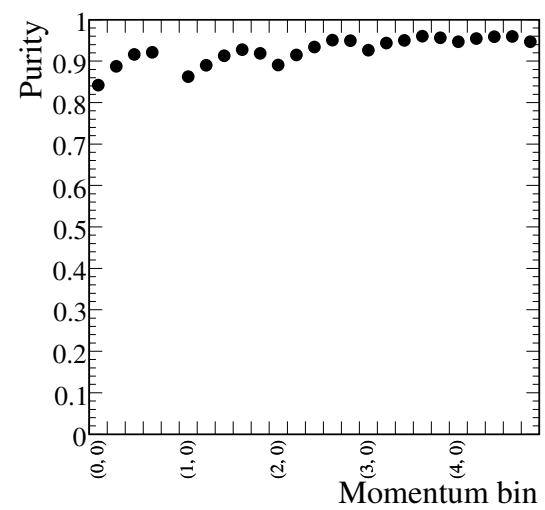

$\mathrm{K}^{+}$

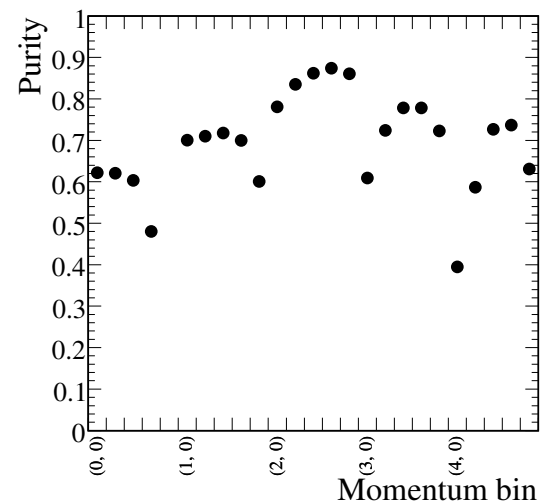

$\mathbf{p}^{+}$

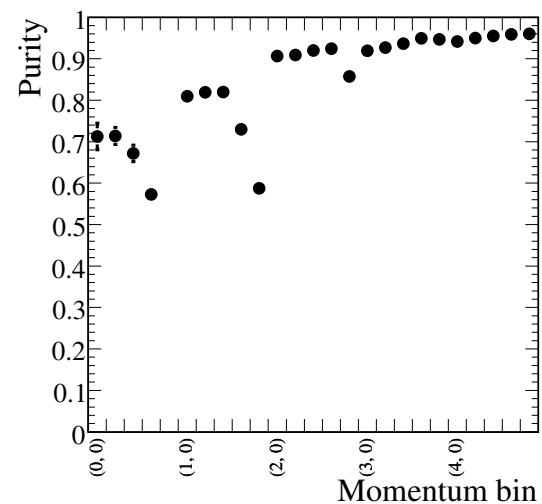

$\pi^{-}$

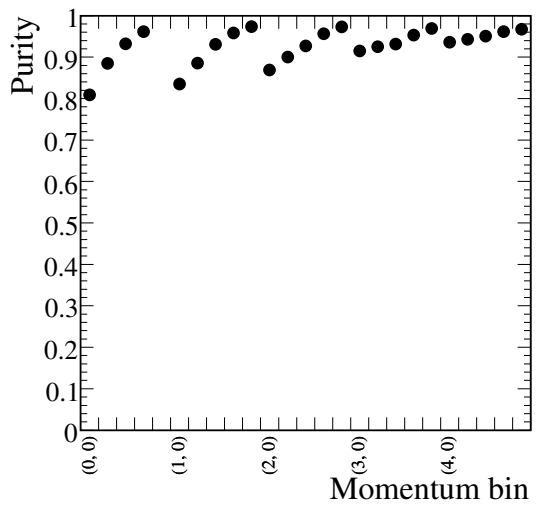

$\mathrm{K}^{-}$

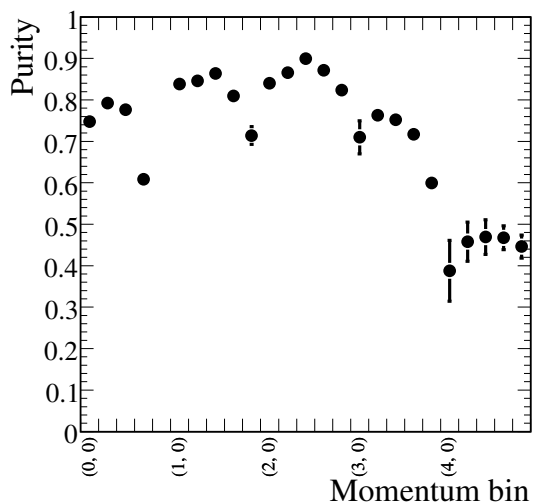

$\mathbf{p}^{-}$

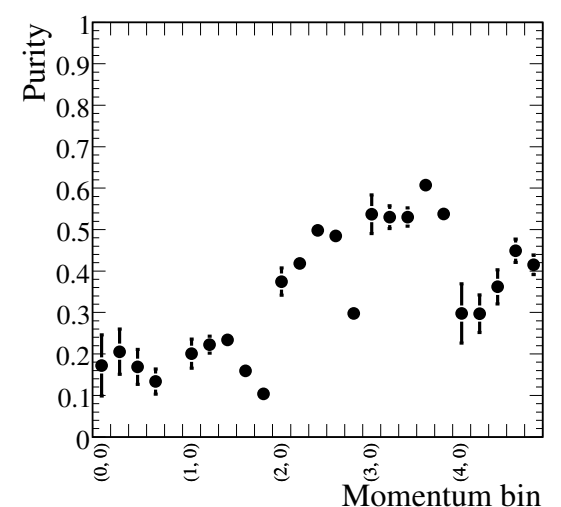

Figure 5.9: Purity of $\pi^{ \pm}, K^{ \pm}$and $p^{ \pm}$as a function of reconstructed momentum bin $\left(p_{z}, p_{T}\right)$. 


\subsubsection{Momentum Correction}

The tracking and fitting procedure outlined in Section 4.2 results in a momentum resolution of $\sim 5 \%$ for $120 \mathrm{GeV} / c$ Monte Carlo protons. It is therefore necessary to unfold the reconstructed momentum distribution measured in data to true momentum distribution in order to calculate the production ratios.

This is achieved by using the Monte Carlo to calculate the probability density function (PDF) for true momentum for each bin of reconstructed momentum. In other words, the true momentum probability distribution is constructed for all particles reconstructed in a single reconstructed momentum bin. These PDF's can be represented as a matrix as shown in Figure 5.10. This matrix is used to reassign the number of events in each reconstructed momentum bin into a range of true bins. The result is a prediction of the true momentum distribution.

This can be summarized mathematically as follows

$$
\begin{gathered}
B^{j}=\sum_{i=1}^{n b i n s} \mathcal{M}_{x}^{i j} b^{i} \\
\sum_{j=1}^{n b i n s} \mathcal{M}_{x}^{i j}=1
\end{gathered}
$$

where $B^{j}=$ number of events in true bin $j, b^{i}=$ number of events in reconstructed bin $i$, and $\mathcal{M}_{x}^{i j}$ is the number of $x$ to assigned to true momentum bin $j$ for each event observed in reconstructed momentum bin $i$. Note that $\mathcal{M}$ is the matrix shown in Figure 5.10. It can be seen that the matrix has only very small non-diagonal elements, reflecting the fact that the momentum resolution is small compared to the 

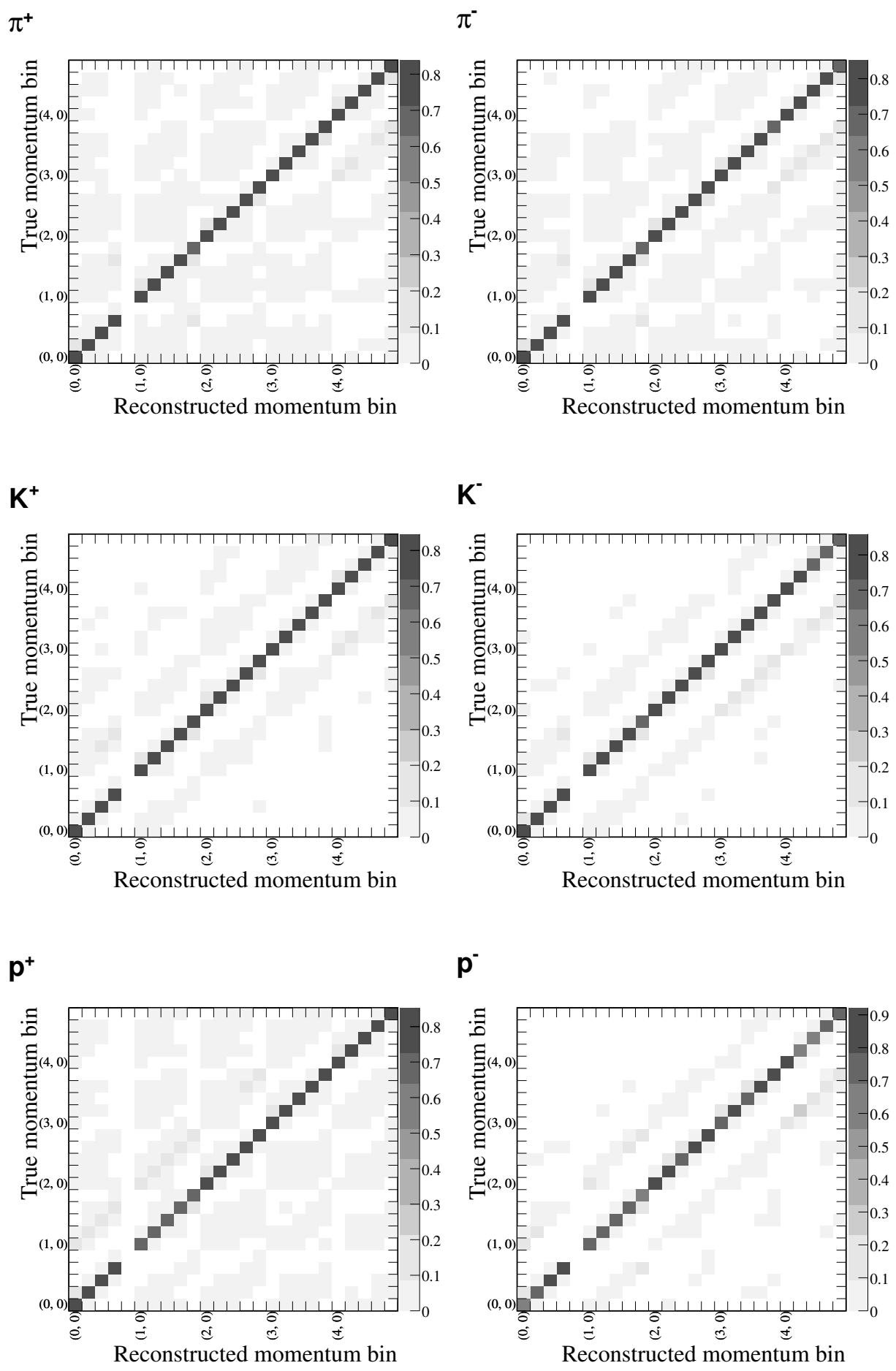

Figure 5.10: Momentum correction factors of $\pi^{ \pm}, K^{ \pm}$and $p^{ \pm}$as a function of true momentum bin versus reconstructed momentum bin. The shaded areas indicate the probability that a reconstructed momentum bin falls into the corresponding true momentum bin. 
bin sizes chosen for the analysis.

\subsubsection{Efficiency Correction}

The identification efficiency of $x$ is defined as

$$
\mathcal{E}_{x}^{j}=\frac{t_{x}^{x}}{T_{x}}
$$

where $T_{x}$ is the number of true $x$ in the Monte Carlo simulation. This is defined per true momentum bin $j$ and is shown in Figure 5.11. The efficiency correction applied bin-by-bin to the data is then $1 / \mathcal{E}$.

\subsubsection{Application of Corrections}

After obtaining the correction factors from the Monte Carlo simulation as described in the previous sections, these can then be applied to the reconstructed mo-

mentum distributions from data. Define $n_{x}^{i}$ to be the number of reconstructed $x$ in reconstructed momentum bin $i$ and $N_{x}^{j}$ to be the predicted number of true $x$ in true momentum bin $j$, obtained by applying the correction factors. Then,

$$
N_{x}^{j}=\sum_{i=1}^{n b i n s} \frac{\mathcal{P}_{x}^{i}}{\mathcal{E}_{x}^{j}} \mathcal{M}_{x}^{i j} n_{x}^{i}
$$

\subsubsection{Background Estimation from Data}

So far, the Monte Carlo has been used to estimate these backgrounds and inefficiencies. However, a major problem with this determination lies in the discrepancy 

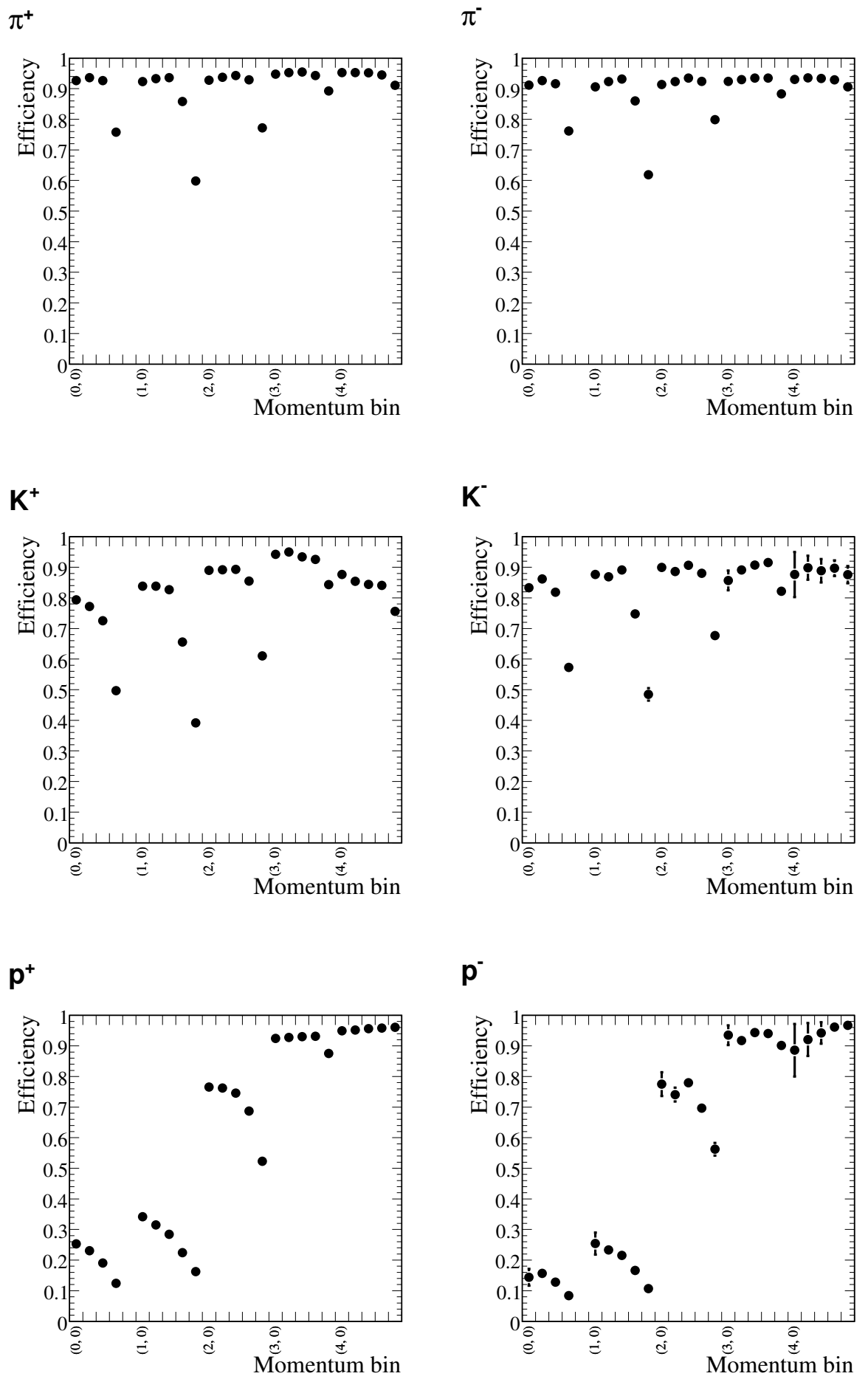

Figure 5.11: Efficiency of $\pi^{ \pm}, K^{ \pm}$and $p^{ \pm}$selection as a function of true momentum bin $\left(p_{z}, p_{T}\right)$. 
between the data and the Monte Carlo simulation. In order to improve the accuracy of the Monte Carlo simulation, an iterative, data-driven approach is adopted. Define a yield for each particle species and each true momentum bin; for example, $f_{\pi^{+}}$for $\pi^{+}$is given by

$$
f_{\pi^{+}}=\frac{N_{\pi^{+}}}{N_{\pi^{+}}+N_{K^{+}}+N_{p^{+}}}
$$

where $N_{x^{+}}$is the predicted number of true $x^{+}$obtained by applying the corrections to the data. Similarly, the yield $F_{\pi^{+}}$for $\pi^{+}$is extracted from the Monte Carlo truth information

$$
F_{\pi^{+}}=\frac{T_{\pi^{+}}}{T_{\pi^{+}}+T_{K^{+}}+T_{p^{+}}}
$$

where $T_{x^{+}}$is the true number of $x^{+}$in the Monte Carlo simulation. Note that for $\pi^{-}$, $K^{-}$and $p^{-}$, the denominator is the sum of the negative $N_{x^{-}}$or $T_{x^{-}}$. Figure 5.12 shows $f_{\pi^{ \pm}}, f_{K^{ \pm}}, f_{p^{ \pm}}$in solid lines and $F_{\pi^{ \pm}}, F_{K^{ \pm}}$, and $F_{p^{ \pm}}$in dashed lines before iteration.

The data/MC yield ratio, $w_{j}=f_{j} / F_{j}$, for particle $j$ is then obtained and used to adjust the Monte Carlo simulation. The yields have no effect on the selection efficiency, and so this tuning only concerns the selection purity.

After weighting each Monte Carlo particle according to the calculated $w_{j}$, the purity correction is recalculated as described in Subsection 5.2.1. The new purity correction, which represents a new mixing scheme between different particle species, is used to calculate new yields from the data and thus new yield ratios.

The evolution of the iteration and the resulting yield ratios are shown in Figure 5.13, where the ratio is shown as a function of momentum bin $\left(p_{z}, p_{T}\right)$ for 7 

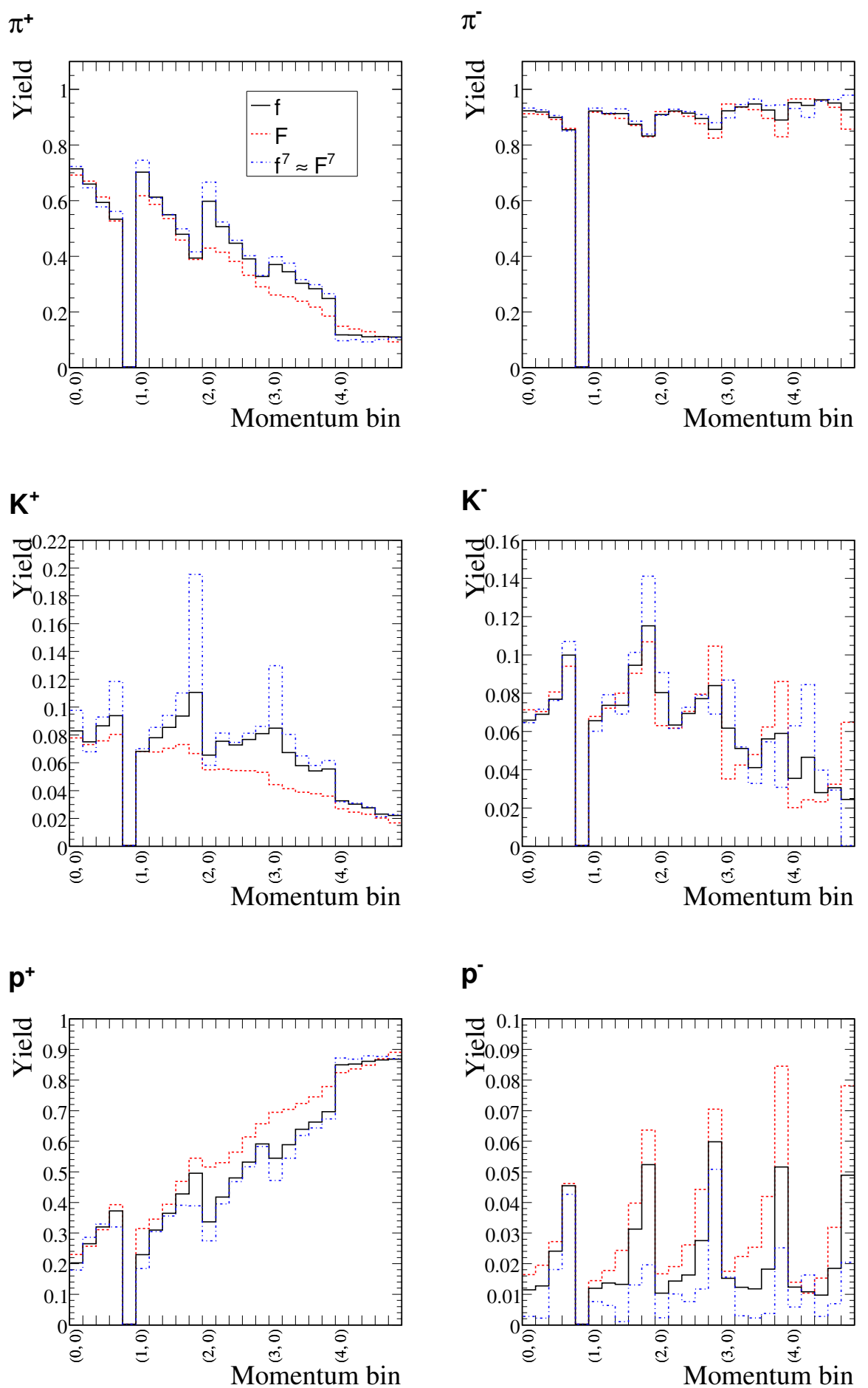

Figure 5.12: Yield for each particle species in data $\left(f_{\pi^{ \pm}}, f_{K^{ \pm}}, f_{p^{ \pm}}\right)$and in Monte Carlo $\left(F_{\pi^{ \pm}}, F_{K^{ \pm}}\right.$, and $\left.F_{p^{ \pm}}\right)$. Solid lines: data; dashed lines: Monte Carlo without iteration; dot-dashed lines: Monte Carlo after the $7^{\text {th }}$ iteration. 
iterations. The yield ratios come to stable values and approach 1 . The yield values after the $7^{\text {th }}$ iteration, $f_{x^{ \pm}}^{7}\left(\cong F_{x^{ \pm}}^{7}\right)$, are shown as dot-dashed lines in Figure 5.12. The final values are closer but not identical to the original data, reflecting the iterative nature of the procedure. In most cases, the yields are driven beyond the original data values.

Figure 5.14 shows the effect of this background tuning on the purity correction as a function of reconstructed momentum bin $\left(p_{z}, p_{T}\right)$ for 7 iterations. Overall, the purity is increased for $\pi^{ \pm}$and $K^{ \pm}$, while the purity for protons decreases on average.

The result of the Monte Carlo tuning on the reconstructed spectrum can be seen in Figure 5.15. The data and Monte Carlo distributions are normalized to each other by area. The total $\chi^{2}$ between the data and the Monte Carlo for all particles before tuning is 6192.06 and after tuning is 4490.72. In general, therefore, the agreement improves after the iteration stage. That is to say the Monte Carlo weighting factors derived from comparisons of the bin-by-bin truth ratios are able to improve agreement at the reconstructed level. It is clear however, that the current Monte Carlo implementation of the experiment is not sufficient to perfectly describe the data: convergence at the truth level does not lead to convergence at the reconstructed level. In particular, although most of the particle species do show improved agreement, the $\pi^{+}$is somewhat poorer after the iteration, as can be seen from the $\chi 2$ breakdowns in Table 5.2. The remaining discrepancies will therefore have to be accounted for by a systematic error. 


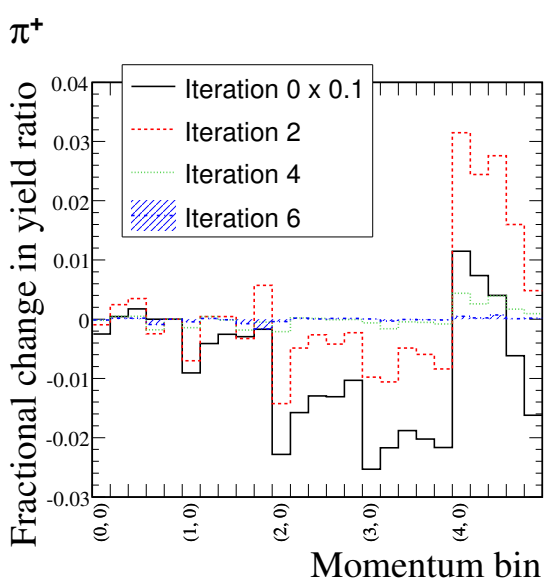

$\pi^{-}$

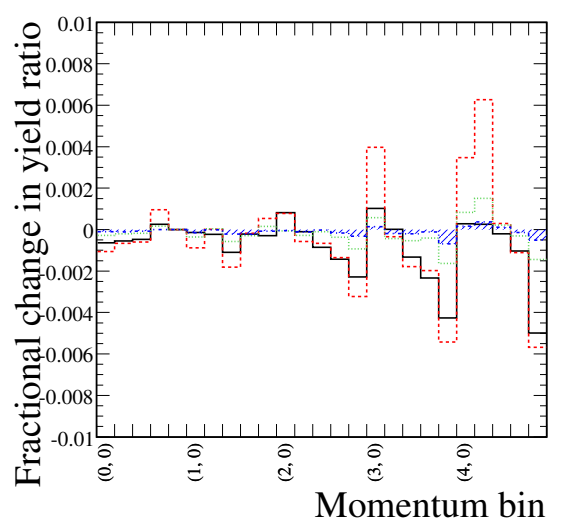

$\mathrm{K}^{+}$

$\mathbf{K}^{-}$
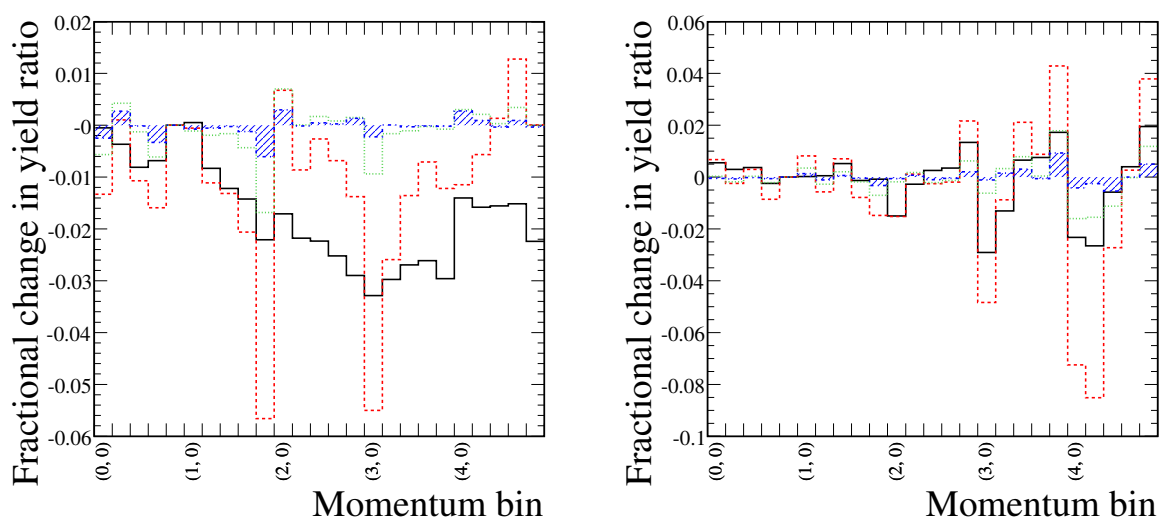

$\mathbf{p}^{+}$

\section{$\mathbf{p}^{-}$}
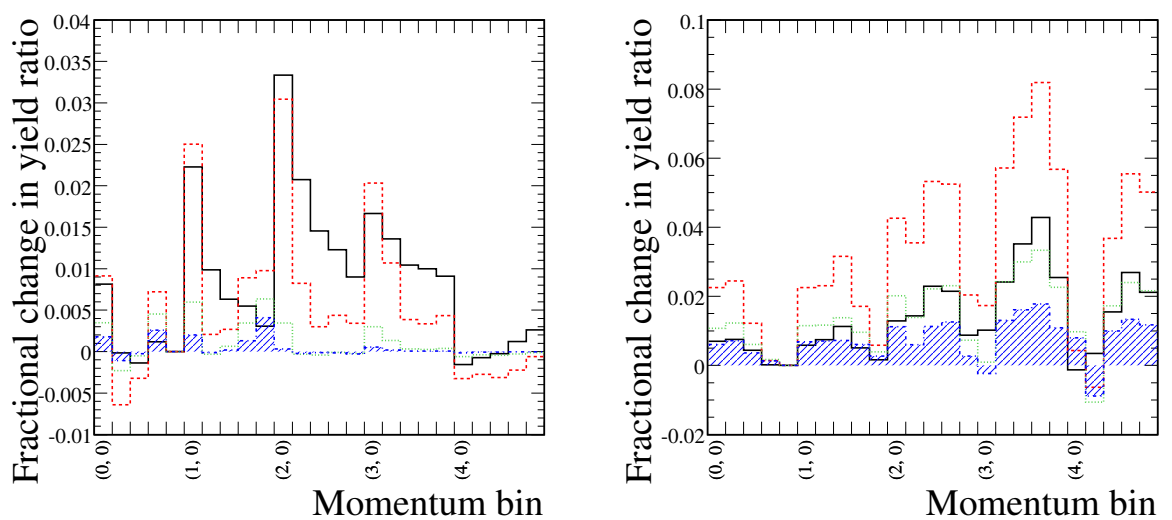

Figure 5.13: Effect of background subtraction on fractional change in yield ratio. The fractional change in yield ratio $\left(w_{j}^{k+1}-w_{j}^{k}\right) / w_{j}^{k}$ between iterations $k$ and $k+1$ for 7 iterations is shown. 

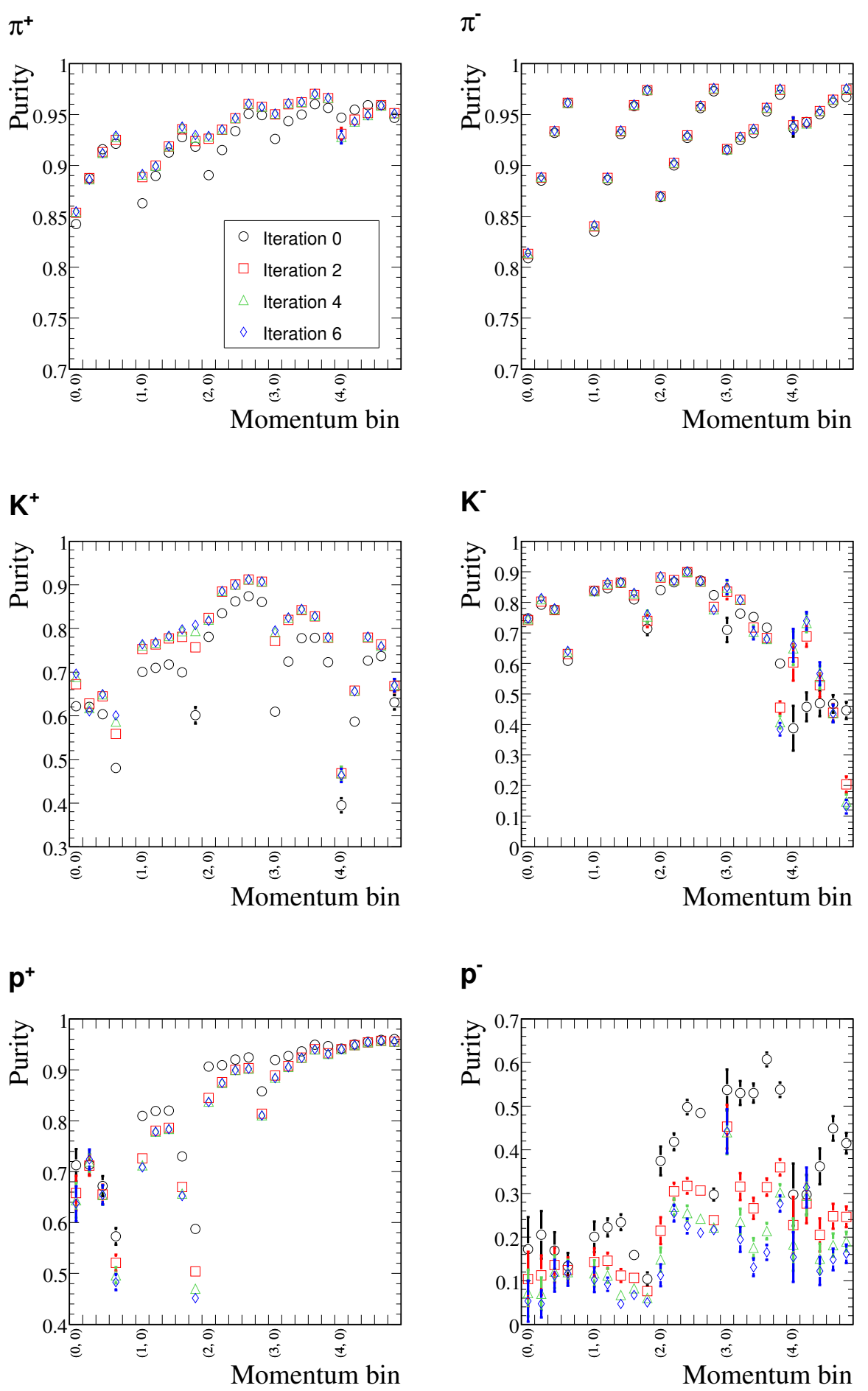

Figure 5.14: Effect of Monte Carlo event weighting on purity correction factor for 7 iterations. 

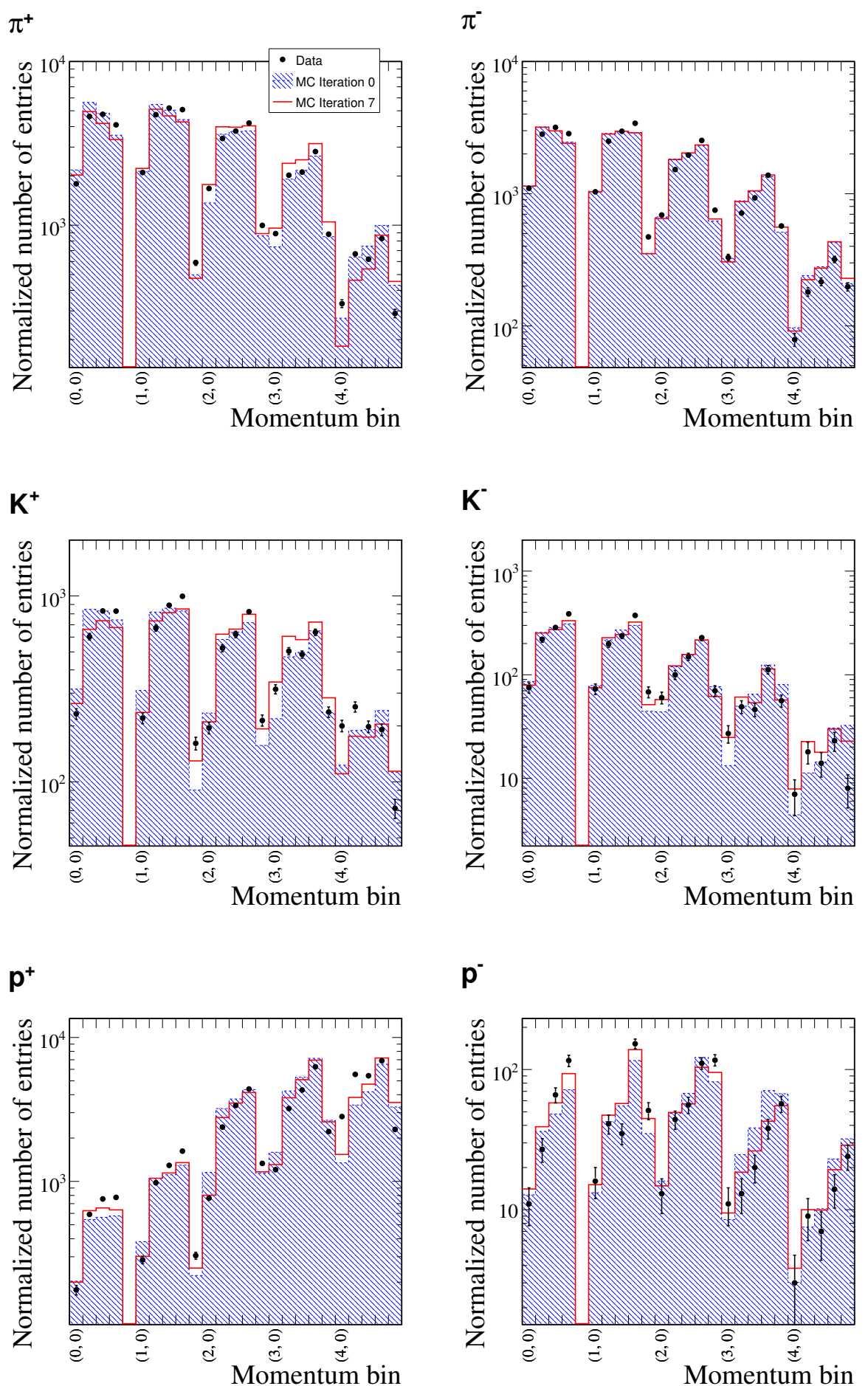

Figure 5.15: Normalized number of entries for $\pi^{ \pm}, K^{ \pm}$and $p^{ \pm}$as a function of momentum bin. Points: data; hatched: Monte Carlo simulation with no iteration; solid lines: Monte Carlo simulation after 7 iterations. 
Table 5.2: The $\chi 2$ between data and Monte Carlo for $\pi^{ \pm}, K^{ \pm}$and $p^{ \pm}$before and after iteration.

\begin{tabular}{c|r|r}
\hline \hline Particle & Before Iteration & After Iteration \\
\hline$\pi^{+}$ & 915.06 & 1109.29 \\
$\pi^{-}$ & 526.26 & 500.59 \\
$K^{+}$ & 423.79 & 270.77 \\
$K^{-}$ & 169.51 & 73.02 \\
$p^{+}$ & 4022.47 & 2492.96 \\
$p^{-}$ & 134.97 & 44.09 \\
\hline Sum & 6192.06 & 4490.72 \\
\hline \hline
\end{tabular}

\subsection{Systematic Errors}

The effect of systematics errors on the $\pi^{-} / \pi^{+}, K^{-} / K^{+}$and $K^{ \pm} / \pi^{ \pm}$ratios have been calculated using the Monte Carlo simulation. An estimation of systematic errors induced by:

- Beam tuning

- Momentum bias

- Background subtraction

are discussed in this section. The total systematic errors are then obtained by quadratic summation of the individual errors from each source.

\subsubsection{Beam Systematic Error}

The goal of this study is to estimate the changes in the production ratio due to small changes in the proton beam profile. This is motivated by observed differ- 
ences between the data and Monte Carlo beam widths. The beam systematic error is obtained by artificially increasing/decreasing the width of the beam after beam selection. Figure 5.16 shows the reconstructed beam positions in data and Monte Carlo; the Monte Carlo was generated with the same beam profile as the NuMI beam in MINOS. The difference in beam width between the MIPP data and the Monte Carlo simulation is noticeable: the Monte Carlo simulation has a wider beam $\Delta \sigma_{x_{\text {beam }}}=\sigma_{x_{\text {beam }}}^{M C}-\sigma_{x_{\text {beam }}}^{\text {data }}=0.1 \mathrm{~mm}$ and $\Delta \sigma y_{\text {beam }}=\sigma_{y_{\text {beam }}}^{M C}-\sigma_{y_{\text {beam }}}^{\text {data }}=0.2 \mathrm{~mm}$.
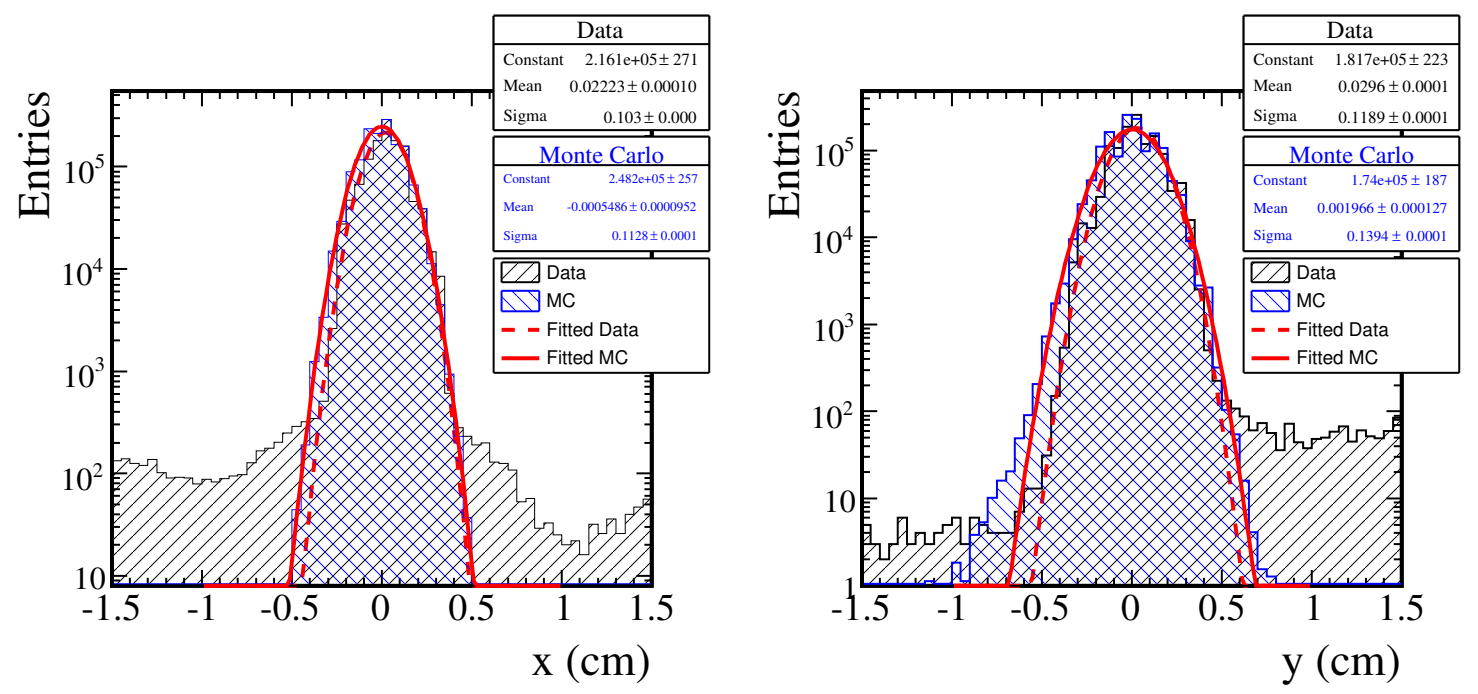

Figure 5.16: Reconstructed beam positions and their Gaussian fits in data and Monte Carlo simulation. Hatched: data and Monte Carlo simulation; dotted lines: Gaussian fit of data; solid lines: Gaussian fit of MC.

These values are used to reweight the Monte Carlo to investigate the effect of a wider/narrower beam. The resulting effects are shown in Figure 5.17 as a function of momentum bin $\left(p_{z}, p_{T}\right)$ for ratios $\pi^{+} / \pi^{-}, K^{+} / K^{-}, K^{+} / \pi^{+}$and $K^{-} / \pi^{-}$. The systematic effect due to beam width variation on the ratios is very small, $<4 \%$ in 
general.

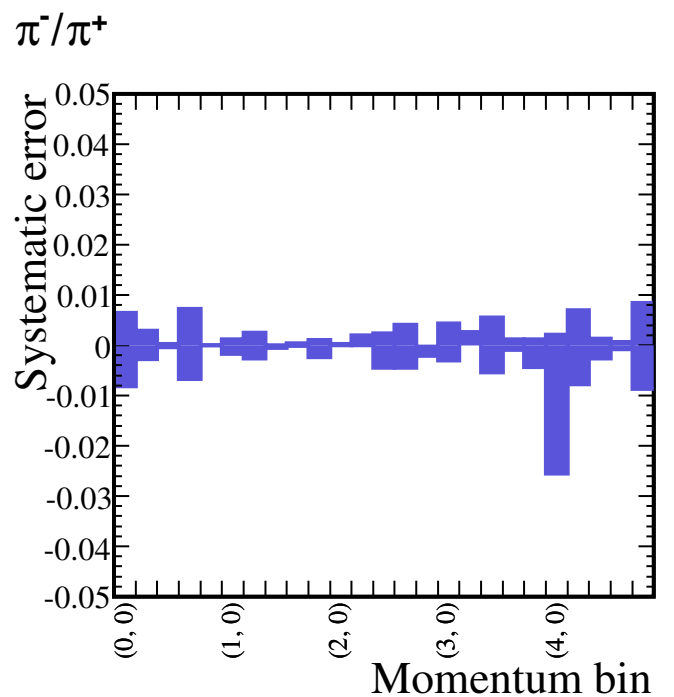

$\mathbf{K}^{-} / \mathbf{K}^{+}$

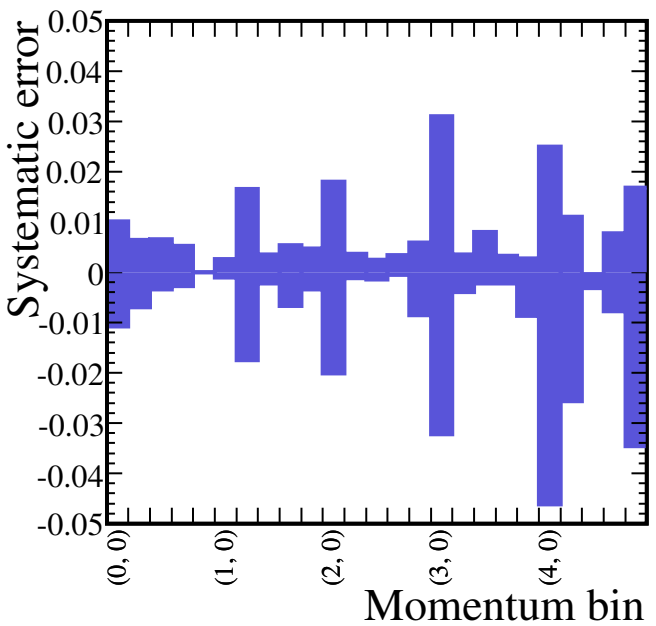

$\mathrm{K}^{+} / \pi^{+}$

$\mathbf{K}^{-} / \pi^{-}$
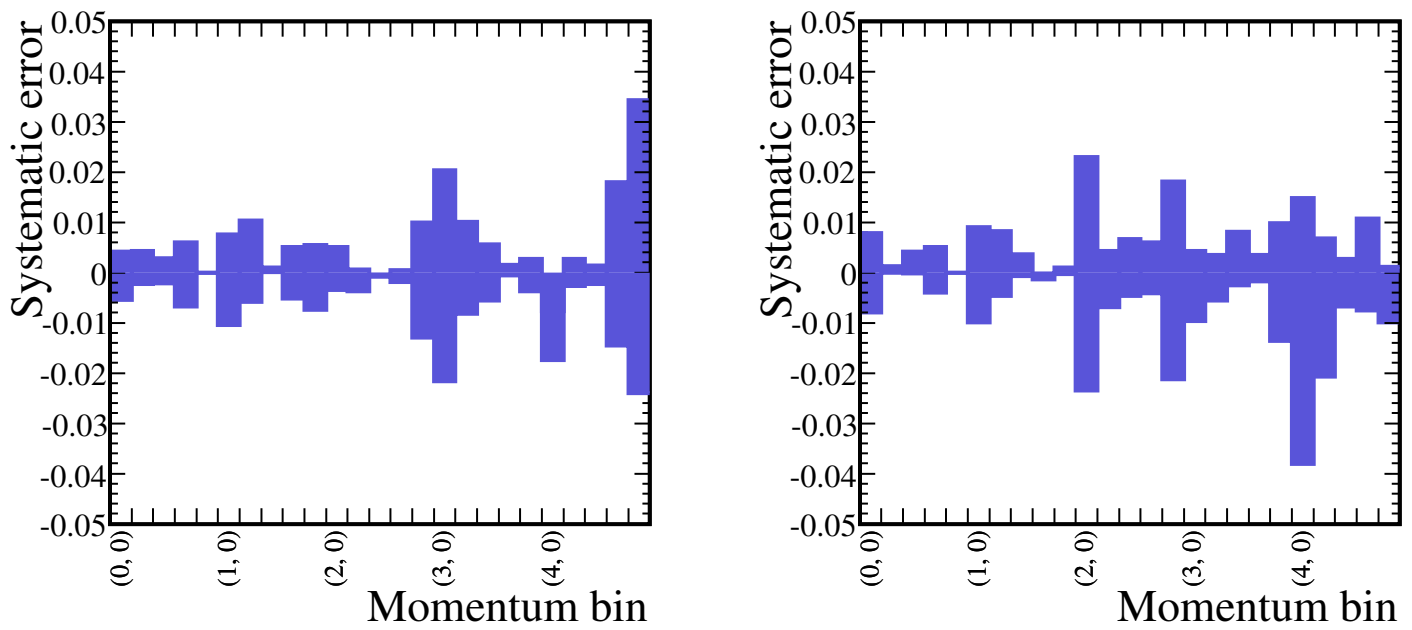

Figure 5.17: Effect of beam systematic on $\pi^{+} / \pi^{-}$(upper left), $K^{+} / K^{-}$(upper right), $K^{+} / \pi^{+}$(lower left) and $K^{-} / \pi^{-}$(lower right) due to $\Delta \sigma_{x_{\text {beam }}}= \pm 0.1 \mathrm{~mm}$ and $\Delta \sigma_{y_{\text {beam }}}= \pm 0.2 \mathrm{~mm}$. 


\subsubsection{Momentum Systematic Error}

The derivation in Subsection 5.2.2 is calculated using the Monte Carlo simulation and therefore does not taken into account differences between the measured reconstructed momentum in the experimental data and the Monte Carlo. As illustrated in Figure 5.18, the average reconstructed momentum in data is actually smaller than in the Monte Carlo simulation for the $120 \mathrm{GeV} / c$ proton beam by about $3 \%$. This observation is used to define the size of momentum bias to use in the systematic error study. A Monte Carlo sample is re-assigned a biased reconstructed momentum, shifted by $\pm 3 \%$ from the original values. This sample is then treated like data and used along with corrections calculated from the standard Monte Carlo to calculate the production ratios.
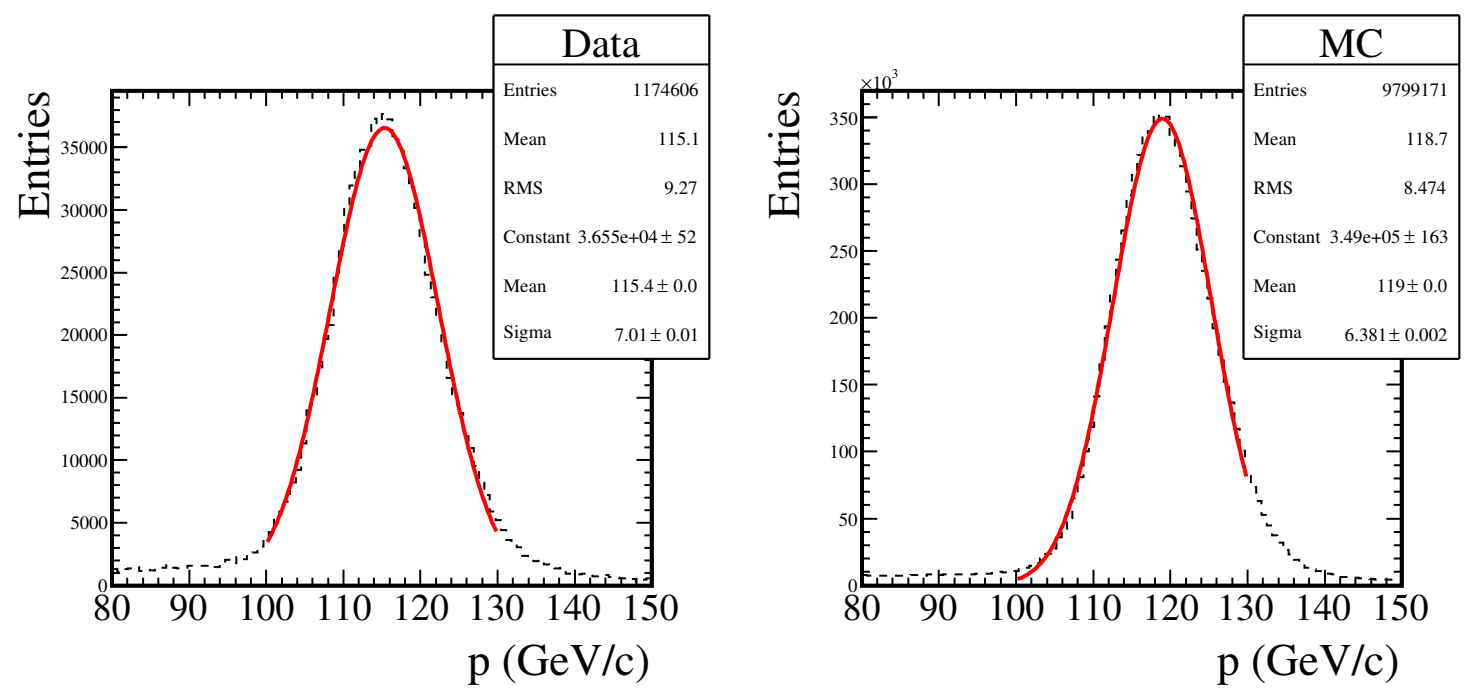

Figure 5.18: Reconstructed momentum and its corresponding Gaussian fit in data (left) and Monte Carlo simulation (right) for $120 \mathrm{GeV} / c$ proton beam. Dotted lines: reconstructed momentum; solid lines: Gaussian fit. 
The resulting effects are shown in Figure 5.19 for ratios $\pi^{+} / \pi^{-}, K^{+} / K^{-}, K^{+} / \pi^{+}$ and $K^{-} / \pi^{-}$as a function of $\left(p_{z}, p_{T}\right)$, by applying a systematic of $\Delta p_{z}= \pm 3 \%$ and $\Delta p_{T}= \pm 3 \%$. The typical effects stay below the $\pm 5 \%$ limit, with at most $15 \%$ effect on a bin per bin basis.

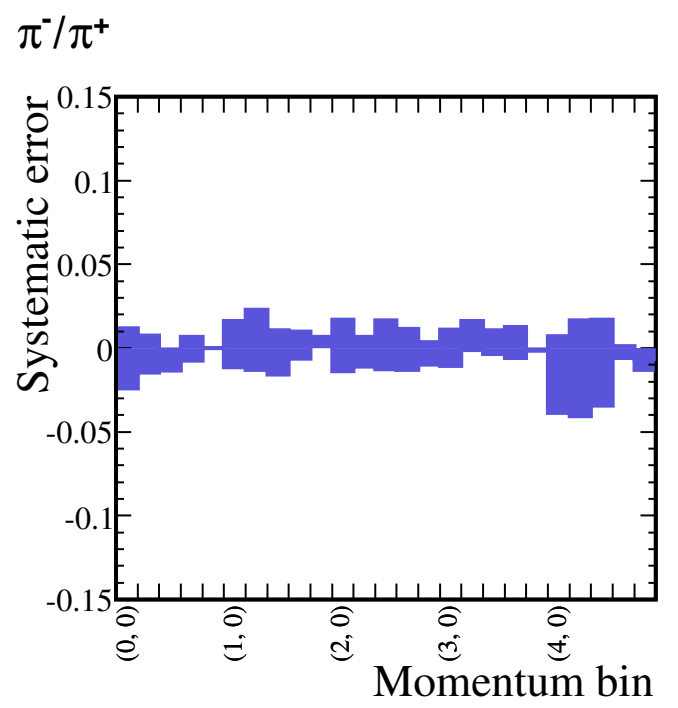

\section{$\mathrm{K}^{-} / \mathrm{K}^{+}$}

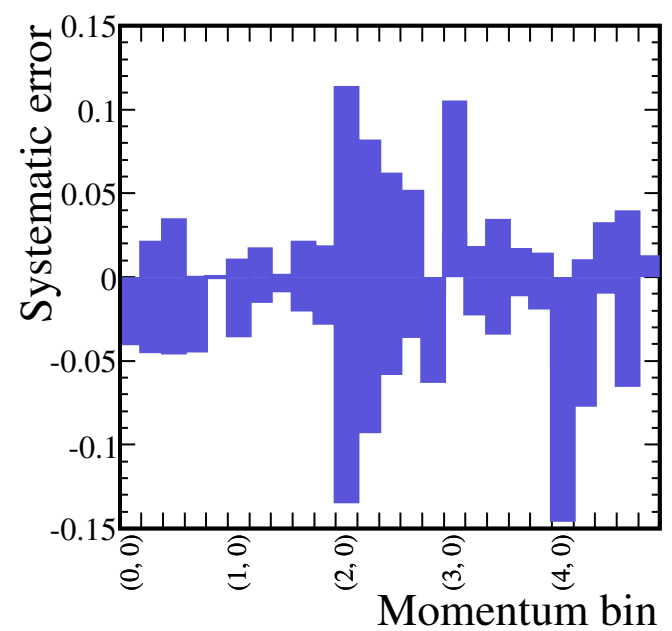

$\mathrm{K}^{+} / \pi^{+}$

$\mathrm{K}^{-} / \pi^{-}$
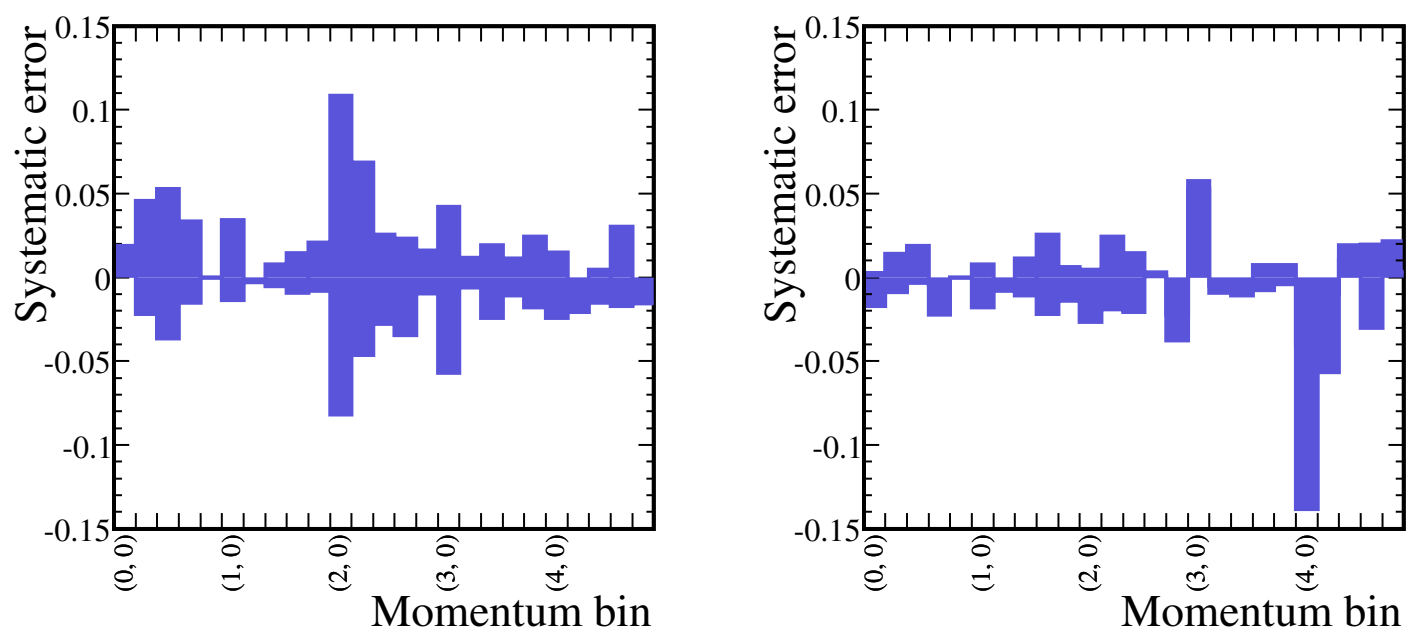

Figure 5.19: Effect of momentum systematic on $\pi^{+} / \pi^{-}$(upper left), $K^{+} / K^{-}$(upper right), $K^{+} / \pi^{+}$(lower left) and $K^{-} / \pi^{-}$(lower right) due to $\Delta p_{z}= \pm 3 \%$ and $\Delta p_{T}=$ $\pm 3 \%$. 


\subsubsection{Background Systematic Error}

The main systematic error is expected to come from the background subtraction. As seen in Subsection 5.2.5, the log-likelihood ratio distributions show some discrepancies between data and Monte Carlo. This can be interpreted as due to a mis-modeling of the background in the Monte Carlo which then leads to differences in the particle yields. The iterative method employed attempts to correct the Monte Carlo for this based on the data measurements. However, the systematic uncertainty introduced through background subtraction depends on the log-likelihood ratio cuts and the accuracy of the purity, and these have not yet been taken into account.

To estimate the systematic error from purity uncertainty after the iterative procedure, the background, $(1-\mathcal{P})$, in each bin is changed by $\pm 10 \%$. The purities are then recomputed for two cases:

$$
\begin{aligned}
& \mathcal{P}^{\prime}=1.1 \mathcal{P}-0.1 \\
& \mathcal{P}^{\prime}=0.9 \mathcal{P}+0.1
\end{aligned}
$$

where $\mathcal{P}^{\prime}$ is the new purity. The predicted production ratios are then calculated from the Monte Carlo using the new purities and the usual analysis chain. The effect of this study is demonstrated in Figure 5.20. It can be seen that the systematic error is typically less than $20 \%$, with large effects on the ratios occuring in bins where the yields are small.

As mentioned in Subsection 5.1.4, the log-likelihood ratio cuts were tuned to give 

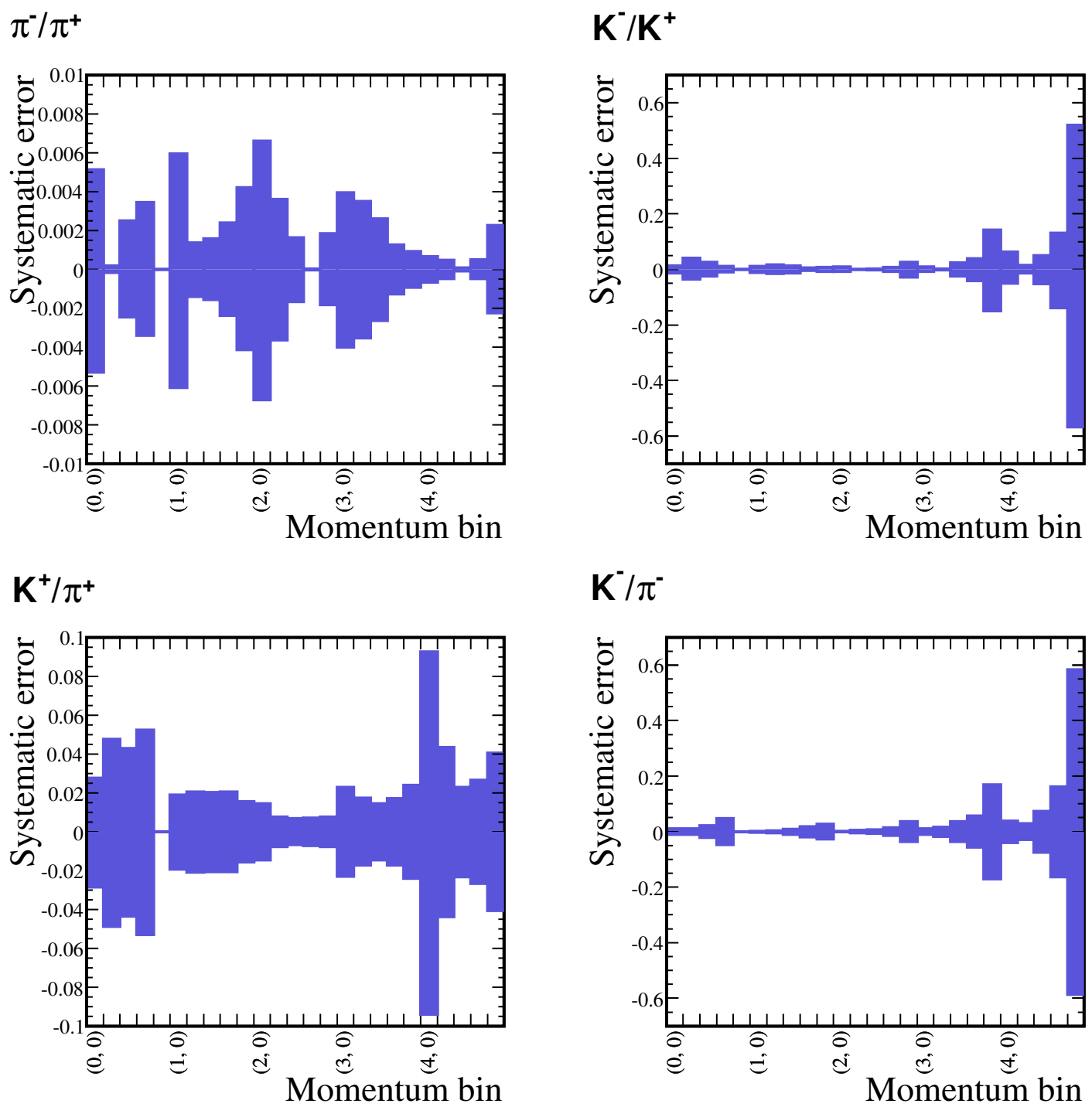

Figure 5.20: Effect of background systematic on $\pi^{+} / \pi^{-}, K^{+} / K^{-}, K^{+} / \pi^{+}$and $K^{-} / \pi^{-}$ due to $\Delta(1-\mathcal{P})= \pm 10 \%$. 
the best compromise between high efficiency and high purity. After the iterations, in order to optimize purity and efficiency, the log-likelihood ratio cuts are found to be different from the original cuts. Here are the new log-likelihood ratio cuts after the iterations:

- $R_{\pi^{+} p^{+}}>5$, select $\pi^{+}$

- $R_{K^{+} p^{+}}>30$, select $K^{+}$

- $R_{K^{-} p^{-}}>10$, select $K^{-}$

It can be seen that the new cut values are lower than the original values by 5 . The optimal log-likelihood cuts are found by varying the cut values in steps of 5 , therefore in order to estimate the systematic error from the cuts, the iteration analysis is redone with a change in each of the cuts of \pm 10 . Figure 5.21 shows the background systematic error from this study as a function of momentum bin $\left(p_{z}, p_{T}\right)$. Similar to the systematic error due to purity uncertainty, the systematic error due to change in log-likelihood ratio cuts is within $20 \%$ in general, with large effects on the bins where the particle yields are small. 

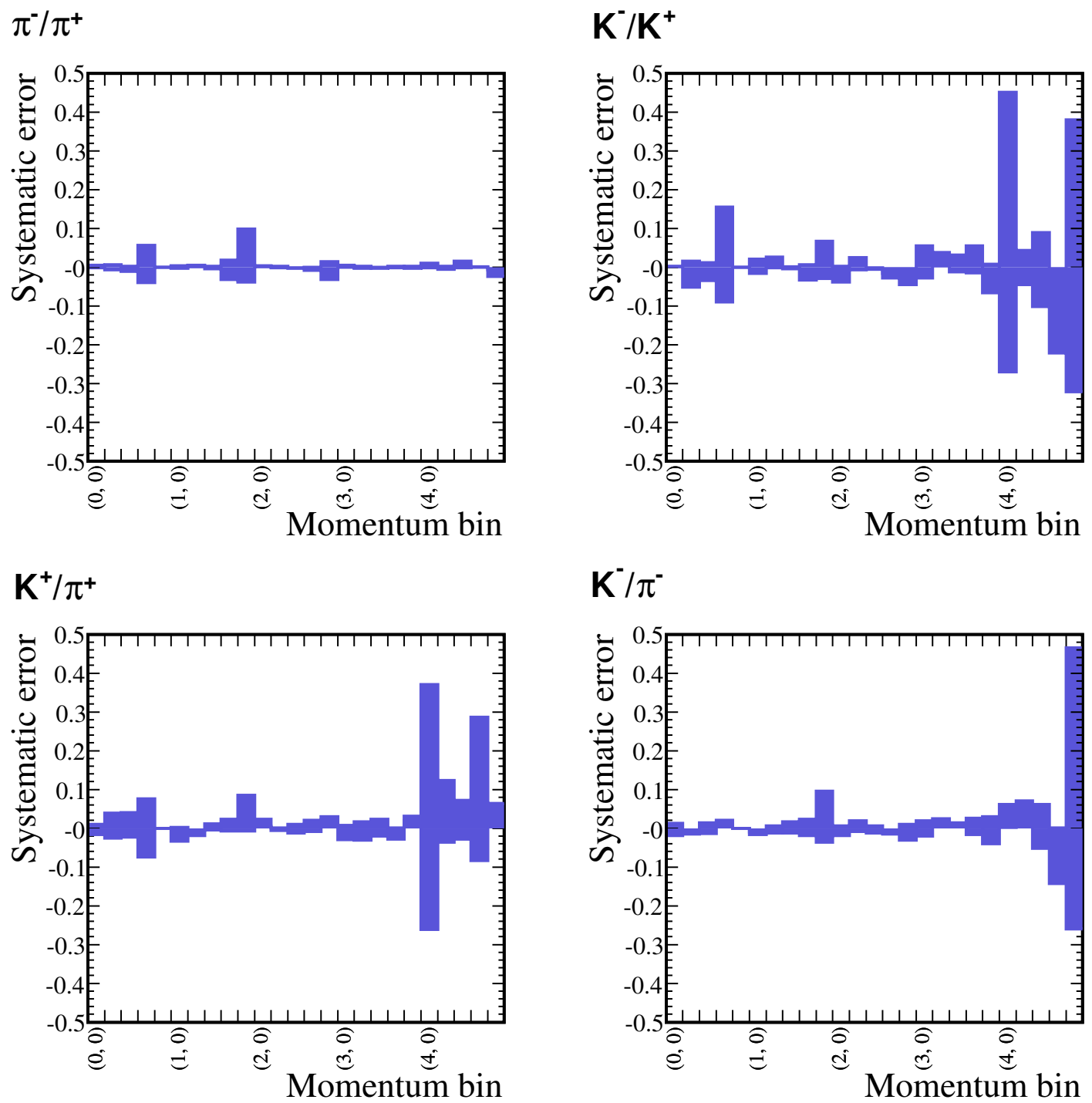

Figure 5.21: Effect of background systematic on $\pi^{+} / \pi^{-}, K^{+} / K^{-}, K^{+} / \pi^{+}$and $K^{-} / \pi^{-}$ due to change in log-likelihood ratio cuts by \pm 10 . 


\section{Chapter 6}

\section{Results and Comparison}

The set of $\pi^{-} / \pi^{+}, K^{-} / K^{+}, K^{ \pm} / \pi^{ \pm}$ratios obtained from the data analysis and correction procedures described in Chapter 5 is shown in this chapter as a function of momentum bin $\left(p_{z}, p_{T}\right)$. This chapter also summarizes the numerical results from the MIPP NuMI target data in tables, gives a set of distributions as a function

of momentum bin $\left(p_{z}, p_{T}\right)$, and shows the comparison to the MIPP Monte Carlo simulation, the MIPP thin Carbon data and the MINOS models.

\subsection{Measurements of Particle Production Ratios from the NuMI Target}

Table 6.1, Table 6.2, Table 6.3 and Table 6.4 present the $\pi^{-} / \pi^{+}, K^{-} / K^{+}, K^{+} / \pi^{+}$ and $K^{-} / \pi^{-}$ratios respectively. They correspond to the binning scheme discussed in 
Table 5.1 in Chapter 5.

The distributions of the ratios in $\left(p_{z}, p_{T}\right)$ are shown in Figure 6.1.
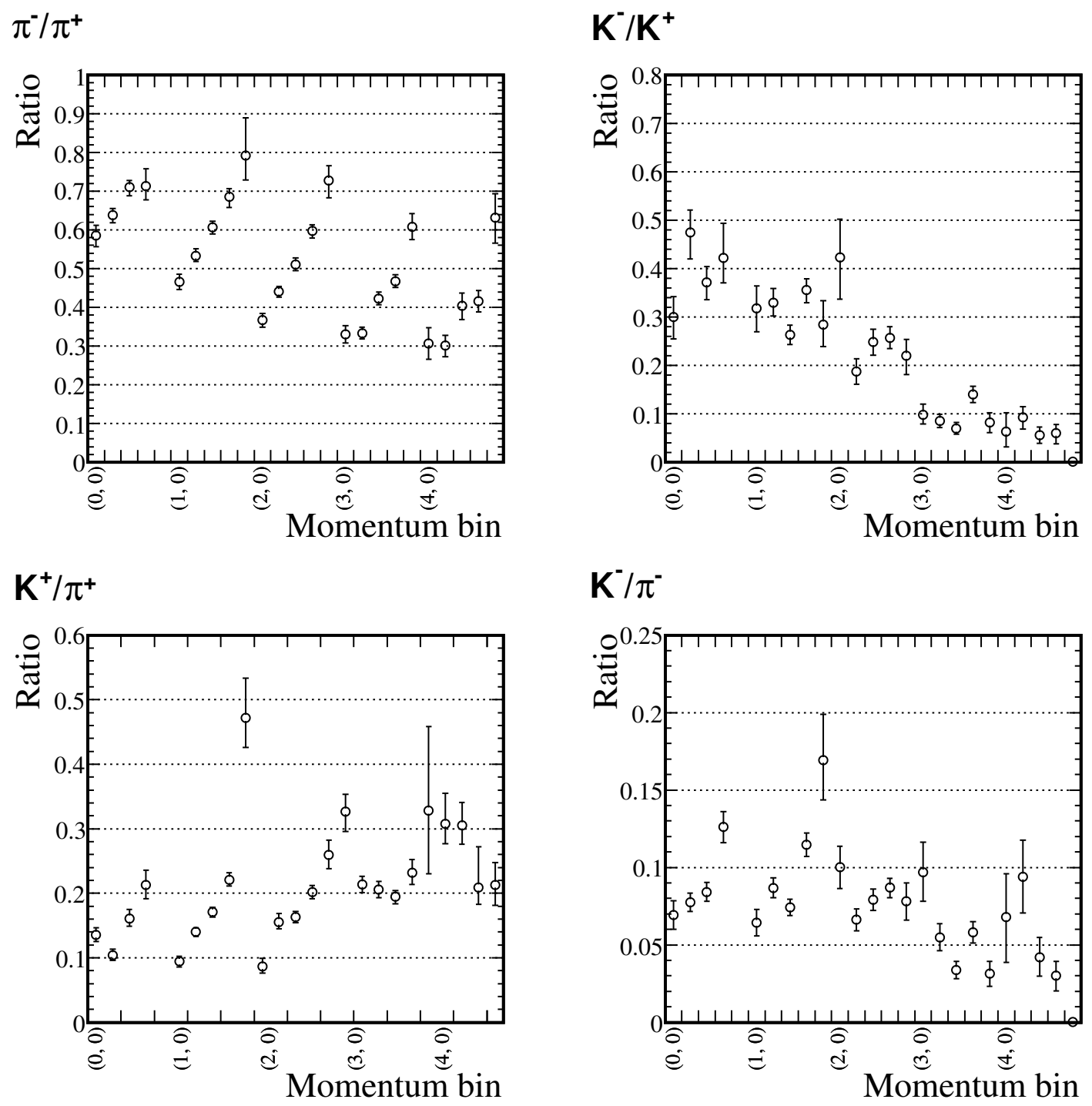

Figure 6.1: The ratios as a function of $\left(p_{z}, p_{T}\right)$ for $\pi^{-} / \pi^{+}, K^{-} / K^{+}$and $K^{ \pm} / \pi^{ \pm}$ produced in $\mathrm{p}+\mathrm{NuMI}$ collisions at $120 \mathrm{GeV} / c$.

These ratios form an internally consistent ensemble that reveals structures of transverse and longitudinal momentum dependencies in the available phase space. 
Table 6.1: The $\pi^{-} / \pi^{+}$ratio in $\mathrm{p}+\mathrm{NuMI}$ interactions in $\left(p_{z}, p_{T}\right)$ at $120 \mathrm{GeV} / c$. The statistical and systematic uncertainties are given.

\begin{tabular}{|c|c|c|c|c|c|c|c|}
\hline Bin & $\pi^{-} / \pi^{+}$ & 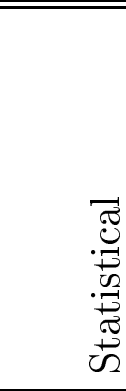 & 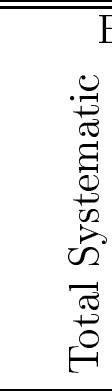 & 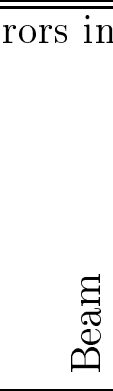 & 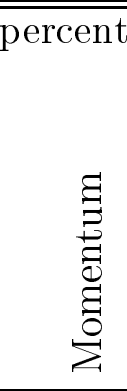 & 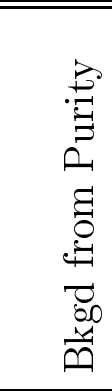 & 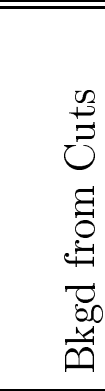 \\
\hline $\begin{array}{l}(0,0) \\
(0,1) \\
(0,2) \\
(0,3)\end{array}$ & $\begin{array}{l}0.585_{-0.029}^{+0.026} \\
0.638_{-0.019}^{+0.017} \\
0.710_{-0.021}^{+0.018} \\
0.713_{-0.035}^{+0.045}\end{array}$ & $\begin{array}{l}4.111 \\
2.531 \\
2.461 \\
2.669\end{array}$ & $\begin{array}{l}+1.506 \\
-2.622 \\
+1.039 \\
-1.623 \\
+0.360 \\
-1.686 \\
+5.745 \\
-4.138\end{array}$ & $\begin{array}{l}+0.638 \\
-0.820 \\
+0.294 \\
-0.276 \\
+0.024 \\
-0.045 \\
+0.722 \\
-0.671\end{array}$ & $\begin{array}{l}+1.170 \\
-2.427 \\
+0.750 \\
-1.454 \\
+0.000 \\
-1.328 \\
+0.665 \\
-0.746\end{array}$ & $\begin{array}{l}+0.513 \\
-0.530 \\
+0.017 \\
-0.017 \\
+0.249 \\
-0.244 \\
+0.345 \\
-0.340\end{array}$ & $\begin{array}{l}+0.478 \\
-0.185 \\
+0.656 \\
-0.667 \\
+0.258 \\
-1.009 \\
+5.649 \\
-4.000\end{array}$ \\
\hline $\begin{array}{l}(1,0) \\
(1,1) \\
(1,2) \\
(1,3) \\
(1,4)\end{array}$ & $\begin{array}{l}0.465_{-0.019}^{+0.020} \\
0.533_{-0.015}^{+0.018} \\
0.607_{-0.016}^{+0.017} \\
0.685_{-0.027}^{+0.021} \\
0.793_{-0.064}^{+0.096}\end{array}$ & $\begin{array}{l}3.890 \\
2.507 \\
2.322 \\
2.261 \\
7.088\end{array}$ & $\begin{array}{l}+1.747 \\
-1.342 \\
+2.365 \\
-1.329 \\
+1.101 \\
-1.620 \\
+2.051 \\
-3.279 \\
+9.851 \\
-3.918\end{array}$ & $\begin{array}{l}+0.115 \\
-0.171 \\
+0.252 \\
-0.265 \\
+0.004 \\
-0.059 \\
+0.044 \\
-0.019 \\
+0.108 \\
-0.237\end{array}$ & $\begin{array}{l}+1.607 \\
{ }_{-1.143} \\
+2.300 \\
-1.294 \\
+1.052 \\
-1.555 \\
+0.974 \\
-0.644 \\
+0.680 \\
{ }_{-0.000}\end{array}$ & $\begin{array}{l}+0.594 \\
{ }_{-0.609} \\
+0.137 \\
-0.140 \\
+0.157 \\
-0.154 \\
+0.239 \\
-0.236 \\
+0.420 \\
{ }_{-0.414}^{0}\end{array}$ & $\begin{array}{l}+0.325 \\
-0.309 \\
+0.470 \\
-0.000 \\
+0.283 \\
-0.342 \\
+1.789 \\
-3.207 \\
+9.818 \\
-3.889\end{array}$ \\
\hline $\begin{array}{l}(2,0) \\
(2,1) \\
(2,2) \\
(2,3) \\
(2,4)\end{array}$ & $\begin{array}{l}0.366_{-0.018}^{+0.018} \\
0.440_{-0.015}^{+0.014} \\
0.510_{-0.016}^{+0.017} \\
0.597_{-0.018}^{+0.017} \\
0.727_{-0.045}^{+0.039}\end{array}$ & $\begin{array}{l}4.606 \\
3.080 \\
2.805 \\
2.534 \\
5.157\end{array}$ & $\begin{array}{l}+1.857 \\
-1.532 \\
+0.832 \\
-1.180 \\
+1.688 \\
-1.383 \\
+1.219 \\
-1.597 \\
-1.438 \\
-3.339\end{array}$ & $\begin{array}{l}+0.023 \\
-0.003 \\
+0.195 \\
-0.000 \\
+0.241 \\
-0.452 \\
+0.407 \\
-0.450 \\
+0.000 \\
-0.209\end{array}$ & $\begin{array}{l}+1.703 \\
-1.377 \\
+0.670 \\
-1.116 \\
+1.663 \\
-1.273 \\
+1.148 \\
-1.309 \\
+0.370 \\
-0.984\end{array}$ & $\begin{array}{l}+0.662 \\
-0.672 \\
+0.359 \\
+0.364 \\
+0.163 \\
+0.165 \\
+0.004 \\
-0.004 \\
+0.185 \\
{ }_{-0.183}\end{array}$ & $\begin{array}{l}+0.333 \\
-0.000 \\
+0.275 \\
-0.120 \\
+0.000 \\
-0.245 \\
+0.048 \\
-0.796 \\
+1.377 \\
-3.179\end{array}$ \\
\hline $\begin{array}{l}(3,0) \\
(3,1) \\
(3,2) \\
(3,3) \\
(3,4)\end{array}$ & $\begin{array}{l}0.330_{-0.022}^{+0.022} \\
0.333_{-0.014}^{+0.015} \\
0.423_{-0.017}^{+0.017} \\
0.467_{-0.017}^{+0.017} \\
0.608_{-0.034}^{+0.034}\end{array}$ & $\begin{array}{l}6.556 \\
4.293 \\
3.903 \\
3.301 \\
5.527\end{array}$ & $\begin{array}{l}+1.326 \\
-1.191 \\
+1.685 \\
-0.425 \\
+1.226 \\
-0.752 \\
+1.313 \\
-0.634 \\
+0.348 \\
-0.537\end{array}$ & $\begin{array}{l}+0.430 \\
-0.300 \\
+0.266 \\
-0.000 \\
+0.554 \\
-0.534 \\
+0.124 \\
-0.091 \\
+0.119 \\
{ }_{-0.433}\end{array}$ & $\begin{array}{l}+1.091 \\
-1.081 \\
+1.610 \\
-0.103 \\
+1.062 \\
-0.374 \\
+1.274 \\
-0.610 \\
+0.000 \\
{ }_{-0.150}\end{array}$ & $\begin{array}{l}+0.395 \\
-0.399 \\
+0.350 \\
-0.353 \\
+0.261 \\
-0.263 \\
+0.125 \\
-0.126 \\
+0.092 \\
{ }_{-0.091}\end{array}$ & $\begin{array}{l}+0.476 \\
{ }_{-0.030} \\
+0.235 \\
-0.214 \\
+0.000 \\
-0.266 \\
+0.267 \\
{ }_{-0.077} \\
{ }_{-0.313} 0.264\end{array}$ \\
\hline $\begin{array}{l}(4,0) \\
(4,1) \\
(4,2) \\
(4,3) \\
(4,4)\end{array}$ & $\begin{array}{l}0.307_{-0.042}^{+0.040} \\
0.301_{-0.027}^{+0.029} \\
0.403_{-0.034}^{+0.035} \\
0.416_{-0.028}^{+0.028} \\
0.631_{-0.062}^{+0.062}\end{array}$ & $\begin{array}{r}12.837 \\
8.637 \\
8.099 \\
6.735 \\
9.843\end{array}$ & $\begin{array}{l}+1.318 \\
-4.626 \\
+1.821 \\
-4.182 \\
+2.336 \\
-3.433 \\
+0.198 \\
-0.602 \\
+0.877 \\
-2.853\end{array}$ & $\begin{array}{l}+0.216 \\
-2.557 \\
+0.698 \\
-0.779 \\
+0.131 \\
-0.264 \\
+0.069 \\
-0.076 \\
+0.847 \\
-0.870\end{array}$ & $\begin{array}{l}+0.704 \\
-3.851 \\
+1.667 \\
-4.074 \\
+1.693 \\
-3.419 \\
+0.101 \\
-0.594 \\
+0.000 \\
{ }_{-1.321}\end{array}$ & $\begin{array}{l}+0.066 \\
-0.065 \\
+0.047 \\
-0.048 \\
+0.008 \\
+0.008 \\
+0.049 \\
+0.049 \\
+0.227 \\
{ }_{-0.025}\end{array}$ & $\begin{array}{l}+1.091 \\
-0.180 \\
+0.212 \\
-0.533 \\
+1.604 \\
-0.171 \\
+0.147 \\
-0.036 \\
+0.000 \\
-2.364\end{array}$ \\
\hline
\end{tabular}


Table 6.2: The $K^{-} / K^{+}$ratio in $\mathrm{p}+\mathrm{NuMI}$ interactions in $\left(p_{z}, p_{T}\right)$ at $120 \mathrm{GeV} / c$. The statistical and systematic uncertainties are given.

\begin{tabular}{|c|c|c|c|c|c|c|c|}
\hline Bin & $K^{-} / K^{+}$ & 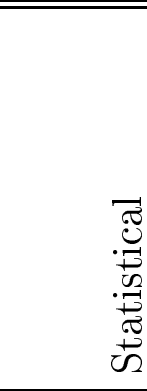 & 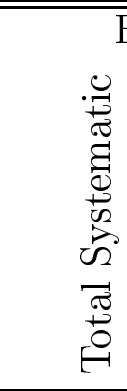 & हี & 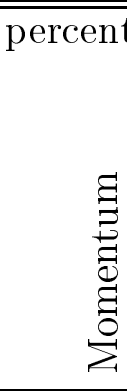 & 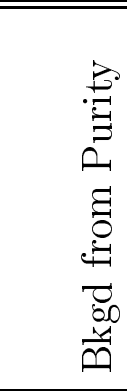 & 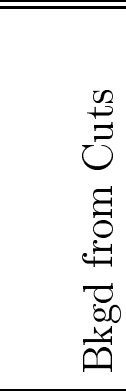 \\
\hline $\begin{array}{l}(0,0) \\
(0,1) \\
(0,2) \\
(0,3)\end{array}$ & $\begin{array}{l}0.299_{-0.045}^{+0.043} \\
0.474_{-0.054}^{+0.047} \\
0.372_{-0.036}^{+0.033} \\
0.422_{-0.052}^{+0.072}\end{array}$ & $\begin{array}{r}14.250 \\
8.520 \\
7.534 \\
7.053\end{array}$ & $\begin{array}{r}+1.702 \\
-4.276 \\
+4.876 \\
-7.668 \\
+4.492 \\
-6.162 \\
+15.503 \\
-9.973\end{array}$ & $\begin{array}{l}+1.007 \\
-1.078 \\
+0.649 \\
-0.695 \\
+0.660 \\
-0.348 \\
+0.520 \\
-0.274\end{array}$ & $\begin{array}{l}+0.000 \\
-3.950 \\
+2.060 \\
-4.416 \\
+3.378 \\
-4.519 \\
+0.000 \\
-4.388\end{array}$ & $\begin{array}{l}+1.348 \\
-1.234 \\
+4.058 \\
-3.593 \\
+2.635 \\
-2.370 \\
+1.008 \\
-0.894\end{array}$ & $\begin{array}{r}+0.257 \\
-0.000 \\
+1.627 \\
-5.090 \\
+1.180 \\
-3.438 \\
+15.461 \\
-8.906\end{array}$ \\
\hline $\begin{array}{l}(1,0) \\
(1,1) \\
(1,2) \\
(1,3) \\
(1,4)\end{array}$ & $\begin{array}{l}0.317_{-0.048}^{+0.047} \\
0.330_{-0.028}^{+0.029} \\
0.263_{-0.020}^{+0.020} \\
0.356_{-0.026}^{+0.023} \\
0.284_{-0.046}^{+0.049}\end{array}$ & $\begin{array}{r}14.615 \\
8.007 \\
7.498 \\
6.171 \\
15.648\end{array}$ & $\begin{array}{l}+2.638 \\
-3.977 \\
+3.908 \\
-2.710 \\
+1.385 \\
-1.559 \\
+2.266 \\
-3.890 \\
+6.932 \\
-4.084\end{array}$ & $\begin{array}{l}+0.256 \\
{ }_{-}^{+0.111} \\
+1.662 \\
+1.748 \\
+0.358 \\
-0.230 \\
+0.538 \\
{ }_{-0.667} \\
{ }_{-0.475}^{+0.475}\end{array}$ & $\begin{array}{l}+1.003 \\
-3.493 \\
+1.663 \\
-1.417 \\
+0.065 \\
-0.798 \\
+2.058 \\
-1.917 \\
{ }_{-2.734} 1.779\end{array}$ & $\begin{array}{l}+1.175 \\
-1.104 \\
+1.604 \\
-1.505 \\
+1.334 \\
-1.258 \\
+0.476 \\
{ }_{-0.451} \\
{ }_{-0.758}^{+0.753}\end{array}$ & $\begin{array}{l}+2.123 \\
-1.545 \\
+2.679 \\
+0.122 \\
+0.083 \\
-0.395 \\
+0.619 \\
-3.287 \\
+6.640 \\
-2.910\end{array}$ \\
\hline $\begin{array}{l}(2,0) \\
(2,1) \\
(2,2) \\
(2,3) \\
(2,4)\end{array}$ & $\begin{array}{l}0.423_{-0.086}^{+0.079} \\
0.187_{-0.026}^{+0.027} \\
0.248_{-0.027}^{+0.027} \\
0.257_{-0.023}^{+0.023} \\
0.220_{-0.039}^{+0.035}\end{array}$ & $\begin{array}{r}14.831 \\
10.894 \\
9.026 \\
7.505 \\
15.536\end{array}$ & $\begin{array}{r}+11.451 \\
-14.072 \\
+8.485 \\
-9.226 \\
+6.106 \\
-5.749 \\
+5.136 \\
-4.548 \\
+2.722 \\
-8.190\end{array}$ & $\begin{array}{l}+1.800 \\
-2.009 \\
+0.370 \\
-0.125 \\
+0.250 \\
+0.145 \\
+0.345 \\
{ }_{-0.054}^{+0.054} \\
{ }_{-0.855}^{+0.595}\end{array}$ & $\begin{array}{r}+11.273 \\
-13.372 \\
+8.106 \\
-9.200 \\
+6.100 \\
-5.723 \\
+5.075 \\
+3.500 \\
+0.000 \\
-6.191\end{array}$ & $\begin{array}{l}{ }_{-0.814}^{+0.851} \\
+0.043 \\
{ }_{-0.044} \\
+0.105 \\
{ }_{-0.108} \\
{ }_{-0.710}^{0.710} \\
{ }_{-0.656}-2.656 \\
{ }_{-2.722}\end{array}$ & $\begin{array}{l}+0.297 \\
-3.809 \\
+2.480 \\
-0.674 \\
+0.000 \\
-0.519 \\
+0.000 \\
+2.811 \\
{ }_{-4}^{+0.000}\end{array}$ \\
\hline $\begin{array}{l}(3,0) \\
(3,1) \\
(3,2) \\
(3,3) \\
(3,4)\end{array}$ & $\begin{array}{c}0.098_{-0.019}^{+0.022} \\
0.085_{-0.014}^{+0.014} \\
0.070_{-0.012}^{+0.012} \\
0.139_{-0.018}^{+0.016} \\
0.082_{-0.021}^{+0.020}\end{array}$ & $\begin{array}{l}19.079 \\
15.873 \\
16.610 \\
10.604 \\
19.365\end{array}$ & $\begin{array}{r}+12.217 \\
-4.329 \\
+4.258 \\
-2.225 \\
+5.249 \\
-4.301 \\
+6.979 \\
-4.455 \\
+14.188 \\
-16.427\end{array}$ & $\begin{array}{l}+3.100 \\
-3.228 \\
+0.355 \\
+0.391 \\
+0.800 \\
+0.228 \\
+0.331 \\
{ }_{-0.221}^{+0.221} \\
{ }_{-0.867}^{+0.273}\end{array}$ & $\begin{array}{r}+10.417 \\
-0.000 \\
+1.737 \\
+2.174 \\
+3.371 \\
+3.329 \\
+1.601 \\
-1.022 \\
{ }_{-1.832} \\
{ }_{-1.821}\end{array}$ & $\begin{array}{r}{ }_{-0.819}^{+0.867} \\
{ }_{-0.256}^{+0.256} \\
{ }_{-0.267}^{+2.323} \\
{ }_{-2.412} \\
+3.931 \\
{ }_{-4.094} \\
{ }_{-14.102}+14.900\end{array}$ & $\begin{array}{l}+5.512 \\
-2.766 \\
+3.863 \\
-0.000 \\
+3.187 \\
+1.246 \\
+5.530 \\
+1.412 \\
{ }_{-6.763}^{+0.763}\end{array}$ \\
\hline $\begin{array}{l}(4,0) \\
(4,1) \\
(4,2) \\
(4,3) \\
(4,4)\end{array}$ & $\begin{array}{l}0.064_{-0.032}^{+0.039} \\
0.092_{-0.022}^{+0.022} \\
0.056_{-0.017}^{+0.017} \\
0.060_{-0.022}^{+0.017} \\
0.001_{-0.002}^{+0.002}\end{array}$ & $\begin{array}{r}40.041 \\
23.730 \\
28.376 \\
25.244 \\
293.522\end{array}$ & $\begin{array}{r}+45.646 \\
-31.385 \\
+4.771 \\
-9.256 \\
+10.666 \\
-11.445 \\
+13.619 \\
-26.855 \\
+64.355 \\
-65.368\end{array}$ & $\begin{array}{l}+2.498 \\
-4.615 \\
+1.107 \\
-2.562 \\
+0.000 \\
-0.321 \\
+0.777 \\
-0.777 \\
+3.684 \\
-3.463\end{array}$ & $\begin{array}{r}+0.000 \\
-14.468 \\
+0.936 \\
+7.602 \\
+3.153 \\
-0.880 \\
+3.865 \\
-6.433 \\
+1.184 \\
{ }_{-0.000}\end{array}$ & $\begin{array}{r}+6.174 \\
-5.045 \\
+1.461 \\
-1.324 \\
+4.960 \\
-5.248 \\
+13.036 \\
-13.864 \\
+51.882 \\
-56.818\end{array}$ & $\begin{array}{r}+45.158 \\
-26.999 \\
+4.304 \\
-4.422 \\
+\quad 8.900 \\
-10.127 \\
+0.000 \\
-22.068 \\
+38.020 \\
-32.136\end{array}$ \\
\hline
\end{tabular}


Table 6.3: The $K^{+} / \pi^{+}$ratio in $\mathrm{p}+\mathrm{NuMI}$ interactions in $\left(p_{z}, p_{T}\right)$ at $120 \mathrm{GeV} / c$. The statistical and systematic uncertainties are given.

\begin{tabular}{|c|c|c|c|c|c|c|c|}
\hline Bin & $K^{+} / \pi^{+}$ & 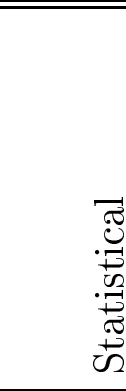 & 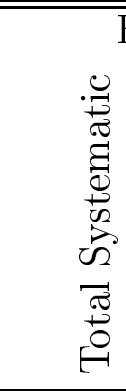 & $\begin{array}{l}\Xi \\
\Phi \\
\oplus\end{array}$ & ह્छ & 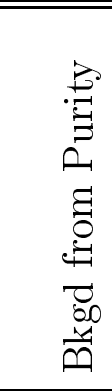 & 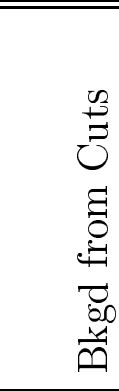 \\
\hline $\begin{array}{l}(0,0) \\
(0,1) \\
(0,2) \\
(0,3)\end{array}$ & $\begin{array}{l}0.136_{-0.011}^{+0.011} \\
0.104_{-0.008}^{+0.009} \\
0.161_{-0.012}^{+0.014} \\
0.213_{-0.022}^{+0.023}\end{array}$ & $\begin{array}{l}7.222 \\
4.926 \\
4.222 \\
4.355\end{array}$ & $\begin{array}{l}+3.514 \\
-3.318 \\
+7.664 \\
-5.888 \\
+7.878 \\
-6.158 \\
+9.796 \\
-9.271\end{array}$ & $\begin{array}{l}+0.412 \\
-0.539 \\
+0.423 \\
-0.226 \\
+0.287 \\
-0.214 \\
+0.595 \\
-0.678\end{array}$ & $\begin{array}{l}+1.860 \\
-0.000 \\
+4.557 \\
-2.172 \\
+5.288 \\
-3.665 \\
+3.326 \\
-1.533\end{array}$ & $\begin{array}{l}+2.757 \\
-2.848 \\
+4.747 \\
-4.869 \\
+4.280 \\
-4.363 \\
+5.219 \\
-5.298\end{array}$ & $\begin{array}{l}+1.057 \\
-1.615 \\
+3.905 \\
-2.488 \\
+3.962 \\
-2.326 \\
+7.570 \\
-7.421\end{array}$ \\
\hline $\begin{array}{l}(1,0) \\
(1,1) \\
(1,2) \\
(1,3) \\
(1,4)\end{array}$ & $\begin{array}{l}0.094_{-0.008}^{+0.008} \\
0.140_{-0.007}^{+0.007} \\
0.171_{-0.008}^{+0.007} \\
0.221_{-0.011}^{+0.001} \\
0.472_{-0.046}^{+0.061}\end{array}$ & $\begin{array}{l}7.430 \\
4.166 \\
3.708 \\
3.597 \\
9.429\end{array}$ & $\begin{array}{l}+3.984 \\
-4.224 \\
+2.294 \\
-2.857 \\
+2.469 \\
-2.181 \\
+3.473 \\
-2.443 \\
+8.889 \\
-2.051\end{array}$ & $\begin{array}{l}+0.756 \\
-1.050 \\
+1.036 \\
-0.581 \\
+0.097 \\
-0.000 \\
+0.513 \\
-0.513 \\
+0.552 \\
-0.736\end{array}$ & $\begin{array}{l}+3.416 \\
-1.362 \\
+0.000 \\
-0.280 \\
+0.765 \\
-0.527 \\
+1.458 \\
-0.908 \\
+2.081 \\
{ }_{-0.812}\end{array}$ & $\begin{array}{l}+1.891 \\
-1.937 \\
+2.047 \\
-2.093 \\
+2.018 \\
-2.054 \\
+2.044 \\
-2.071 \\
+1.530 \\
-1.552\end{array}$ & $\begin{array}{l}+0.231 \\
-3.336 \\
+0.000 \\
+1.835 \\
+1.196 \\
-0.509 \\
+2.344 \\
-0.766 \\
+8.488 \\
-0.774\end{array}$ \\
\hline $\begin{array}{l}(2,0) \\
(2,1) \\
(2,2) \\
(2,3) \\
(2,4)\end{array}$ & $\begin{array}{l}0.087_{-0.010}^{+0.012} \\
0.156_{-0.010}^{+0.013} \\
0.163_{-0.008}^{+0.000} \\
0.202_{-0.010}^{+0.010} \\
0.259_{-0.021}^{+0.023}\end{array}$ & $\begin{array}{l}8.297 \\
4.651 \\
4.371 \\
3.826 \\
8.038\end{array}$ & $\begin{array}{r}+11.180 \\
-8.321 \\
+6.876 \\
-4.736 \\
+2.824 \\
-3.139 \\
+3.178 \\
-3.610 \\
+3.607 \\
-1.780\end{array}$ & $\begin{array}{l}+0.508 \\
-0.352 \\
+0.063 \\
-0.368 \\
+0.000 \\
+0.082 \\
+0.050 \\
-0.195 \\
+0.994 \\
-1.297\end{array}$ & $\begin{array}{l}+10.820 \\
-8.186 \\
+6.833 \\
-4.617 \\
+2.552 \\
-2.794 \\
+2.324 \\
-3.442 \\
+1.592 \\
-0.950\end{array}$ & $\begin{array}{l}+1.428 \\
-1.450 \\
+0.760 \\
-0.770 \\
+0.673 \\
-0.681 \\
+0.709 \\
-0.716 \\
+0.758 \\
-0.764\end{array}$ & $\begin{array}{l}+2.369 \\
-0.000 \\
+0.110 \\
-0.615 \\
+1.006 \\
+1.257 \\
+2.048 \\
+0.795 \\
+0.985 \\
-0.000\end{array}$ \\
\hline $\begin{array}{l}(3,0) \\
(3,1) \\
(3,2) \\
(3,3) \\
(3,4)\end{array}$ & $\begin{array}{l}0.327_{-0.031}^{+0.027} \\
0.214_{-0.012}^{+0.013} \\
0.206_{-0.012}^{+0.012} \\
0.195_{-0.001}^{+0.001} \\
0.232_{-0.019}^{+0.020}\end{array}$ & $\begin{array}{l}6.201 \\
4.843 \\
4.958 \\
4.403 \\
7.427\end{array}$ & $\begin{array}{l}+5.239 \\
-7.143 \\
+2.873 \\
-3.642 \\
+3.408 \\
-3.516 \\
+2.056 \\
-3.467 \\
+4.632 \\
-3.027\end{array}$ & $\begin{array}{l}+2.028 \\
-2.168 \\
+1.010 \\
-0.819 \\
+0.566 \\
-0.563 \\
+0.149 \\
-0.052 \\
+0.269 \\
{ }_{-0.379}\end{array}$ & $\begin{array}{l}+4.195 \\
-5.689 \\
+1.176 \\
-0.607 \\
+1.933 \\
-2.423 \\
+1.143 \\
-1.068 \\
+2.440 \\
-1.804\end{array}$ & $\begin{array}{l}+2.273 \\
-2.298 \\
+1.716 \\
-1.732 \\
+1.444 \\
-1.456 \\
+1.701 \\
-1.712 \\
+2.384 \\
-2.402\end{array}$ & $\begin{array}{l}+0.760 \\
-2.946 \\
+1.705 \\
-3.037 \\
+2.340 \\
-2.014 \\
+0.078 \\
-2.818 \\
+-3.122 \\
-0.000\end{array}$ \\
\hline $\begin{array}{l}(4,0) \\
(4,1) \\
(4,2) \\
(4,3) \\
(4,4)\end{array}$ & $\begin{array}{l}0.328_{-0.098}^{+0.131} \\
0.308_{-0.047}^{+0.031} \\
0.305_{-0.029}^{+0.035} \\
0.209_{-0.02}^{+0.063} \\
0.213_{-0.032}^{+0.035}\end{array}$ & $\begin{array}{r}10.927 \\
8.061 \\
8.799 \\
8.476 \\
13.996\end{array}$ & $\begin{array}{r}+38.253 \\
-27.878 \\
+13.033 \\
-5.999 \\
+7.538 \\
-3.923 \\
+28.965 \\
-9.040 \\
+8.346 \\
-4.970\end{array}$ & $\begin{array}{l}+0.000 \\
-1.737 \\
+0.267 \\
-0.273 \\
+0.140 \\
-0.228 \\
+1.795 \\
-1.458 \\
+3.429 \\
-2.400\end{array}$ & $\begin{array}{l}+1.477 \\
-2.440 \\
+0.000 \\
-2.072 \\
+0.458 \\
-1.528 \\
+3.035 \\
-1.730 \\
+0.000 \\
-1.539\end{array}$ & $\begin{array}{l}+9.258 \\
-9.396 \\
+4.319 \\
-4.370 \\
+2.292 \\
-2.315 \\
+2.639 \\
-2.662 \\
+4.031 \\
-4.071\end{array}$ & $\begin{array}{r}+37.086 \\
-26.076 \\
+12.294 \\
-3.539 \\
+7.166 \\
-2.764 \\
+28.628 \\
-8.338 \\
+6.454 \\
-0.000\end{array}$ \\
\hline
\end{tabular}


Table 6.4: The $K^{-} / \pi^{-}$ratio in $\mathrm{p}+\mathrm{NuMI}$ interactions in $\left(p_{z}, p_{T}\right)$ at $120 \mathrm{GeV} / c$. The statistical and systematic uncertainties are given.

\begin{tabular}{|c|c|c|c|c|c|c|c|}
\hline Bin & $K^{-} / \pi^{-}$ & 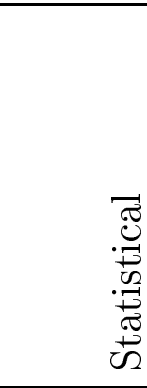 & 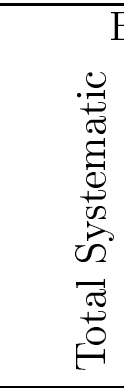 & @ె & 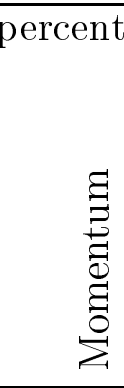 & 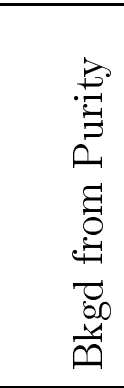 & 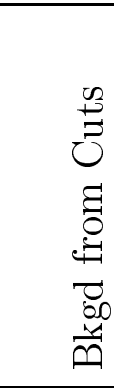 \\
\hline $\begin{array}{l}(0,0) \\
(0,1) \\
(0,2) \\
(0,3)\end{array}$ & $\begin{array}{c}0.069_{-0.009}^{+0.009} \\
0.077_{-0.006}^{+0.006} \\
0.084_{-0.006}^{+0.006} \\
0.126_{-0.010}^{+0.010}\end{array}$ & $\begin{array}{r}12.954 \\
7.398 \\
6.707 \\
6.156\end{array}$ & $\begin{array}{l}+1.825 \\
-2.813 \\
+1.737 \\
-2.063 \\
+3.155 \\
-2.551 \\
+5.102 \\
-5.187\end{array}$ & $\begin{array}{l}+0.780 \\
-0.797 \\
+0.127 \\
-0.000 \\
+0.421 \\
-0.018 \\
+0.514 \\
-0.400\end{array}$ & $\begin{array}{l}+0.270 \\
-1.701 \\
+1.414 \\
-0.900 \\
+1.884 \\
-0.330 \\
+0.000 \\
-2.215\end{array}$ & $\begin{array}{l}+0.972 \\
-1.014 \\
+1.001 \\
-1.026 \\
+2.058 \\
-2.088 \\
+4.634 \\
-4.673\end{array}$ & $\begin{array}{l}+1.306 \\
-1.832 \\
+0.000 \\
-1.547 \\
+1.411 \\
-1.428 \\
+2.072 \\
-0.000\end{array}$ \\
\hline $\begin{array}{l}(1,0) \\
(1,1) \\
(1,2) \\
(1,3) \\
(1,4)\end{array}$ & $\begin{array}{c}0.064_{-0.009}^{+0.009} \\
0.087_{-0.006}^{+0.006} \\
0.074_{-0.005}^{+0.005} \\
0.115_{-0.008}^{+0.008} \\
0.169_{-0.026}^{+0.030}\end{array}$ & $\begin{array}{r}13.173 \\
7.283 \\
6.918 \\
5.500 \\
14.359\end{array}$ & $\begin{array}{l}+1.201 \\
-2.595 \\
+1.095 \\
-1.549 \\
+2.201 \\
-1.839 \\
+3.863 \\
-3.433 \\
+9.991 \\
-4.762\end{array}$ & $\begin{array}{l}+0.898 \\
-0.991 \\
+0.817 \\
-0.466 \\
+0.361 \\
-0.075 \\
+0.000 \\
-0.139 \\
+0.095 \\
-0.027\end{array}$ & $\begin{array}{l}+0.779 \\
-1.774 \\
+0.000 \\
-0.789 \\
+1.129 \\
-1.080 \\
+2.548 \\
-2.178 \\
+0.623 \\
-1.380\end{array}$ & $\begin{array}{l}+0.171 \\
-0.178 \\
+0.374 \\
-0.383 \\
+0.889 \\
-0.902 \\
+1.824 \\
-1.840 \\
+2.720 \\
-2.735\end{array}$ & $\begin{array}{l}+0.000 \\
-1.604 \\
+0.626 \\
-1.188 \\
+1.628 \\
-1.182 \\
+2.259 \\
-1.907 \\
+9.593 \\
-3.645\end{array}$ \\
\hline $\begin{array}{l}(2,0) \\
(2,1) \\
(2,2) \\
(2,3) \\
(2,4)\end{array}$ & $\begin{array}{c}0.100_{-0.014}^{+0.013} \\
0.066_{-0.007}^{+0.007} \\
0.079_{-0.007}^{+0.007} \\
0.087_{-0.006}^{+0.006} \\
0.078_{-0.012}^{+0.012}\end{array}$ & $\begin{array}{r}13.127 \\
10.322 \\
8.380 \\
6.936 \\
14.259\end{array}$ & $\begin{array}{l}+2.430 \\
-4.006 \\
+3.188 \\
-2.234 \\
+1.811 \\
-2.512 \\
+1.573 \\
-2.136 \\
+4.183 \\
-6.401\end{array}$ & $\begin{array}{l}+2.294 \\
-2.352 \\
+0.430 \\
-0.686 \\
+0.659 \\
-0.467 \\
+0.602 \\
-0.410 \\
+1.808 \\
-2.117\end{array}$ & $\begin{array}{l}+0.454 \\
-2.658 \\
+2.427 \\
-1.901 \\
+1.449 \\
-2.071 \\
+0.306 \\
-0.000 \\
+0.000 \\
-3.750\end{array}$ & $\begin{array}{l}+0.060 \\
-0.058 \\
+0.442 \\
-0.452 \\
+0.615 \\
-0.624 \\
+1.421 \\
-1.433 \\
+3.624 \\
-3.643\end{array}$ & $\begin{array}{l}+0.656 \\
-1.857 \\
+1.973 \\
-0.837 \\
+0.605 \\
-1.190 \\
+0.000 \\
-1.530 \\
+1.048 \\
-3.026\end{array}$ \\
\hline $\begin{array}{l}(3,0) \\
(3,1) \\
(3,2) \\
(3,3) \\
(3,4)\end{array}$ & $\begin{array}{c}0.097_{-0.019}^{+0.020} \\
0.055_{-0.009}^{+0.009} \\
0.034_{-0.006}^{+0.006} \\
0.058_{-0.007}^{+0.007} \\
0.031_{-0.008}^{+0.008}\end{array}$ & $\begin{array}{l}19.197 \\
15.714 \\
16.326 \\
10.196 \\
18.719\end{array}$ & $\begin{array}{r}+6.159 \\
-2.458 \\
+2.966 \\
-1.970 \\
+3.876 \\
-3.749 \\
+6.211 \\
-5.890 \\
+17.237 \\
-17.542\end{array}$ & $\begin{array}{l}+0.433 \\
-0.969 \\
+0.348 \\
-0.564 \\
+0.812 \\
-0.257 \\
+0.356 \\
-0.182 \\
+0.980 \\
-1.361\end{array}$ & $\begin{array}{l}+5.743 \\
-0.000 \\
+0.000 \\
-0.922 \\
+0.000 \\
-1.090 \\
+0.724 \\
-0.748 \\
+0.725 \\
-0.409\end{array}$ & $\begin{array}{l}+1.036 \\
-1.056 \\
+1.621 \\
-1.647 \\
+3.530 \\
-3.578 \\
+5.566 \\
-5.617 \\
+16.929 \\
-17.020\end{array}$ & $\begin{array}{l}+1.918 \\
-1.997 \\
+2.459 \\
-0.000 \\
+1.379 \\
-0.000 \\
+2.635 \\
-1.598 \\
+3.008 \\
-4.000\end{array}$ \\
\hline $\begin{array}{l}(4,0) \\
(4,1) \\
(4,2) \\
(4,3) \\
(4,4)\end{array}$ & $\begin{array}{c}0.068_{-0.029}^{+0.028} \\
0.094_{-0.023}^{+0.024} \\
0.042_{-0.012}^{+0.013} \\
0.030_{-0.010}^{+0.009} \\
0.000_{-0.001}^{+0.001}\end{array}$ & $\begin{array}{r}40.604 \\
23.931 \\
28.167 \\
24.714 \\
293.353\end{array}$ & $\begin{array}{r}+7.387 \\
-14.841 \\
+7.696 \\
-6.710 \\
+9.743 \\
-9.115 \\
+16.232 \\
-21.836 \\
+74.696 \\
-64.184\end{array}$ & $\begin{array}{l}+1.485 \\
-3.813 \\
+0.675 \\
-2.065 \\
+0.270 \\
-0.679 \\
+1.080 \\
-0.760 \\
+0.115 \\
-0.992\end{array}$ & $\begin{array}{r}+0.000 \\
-13.812 \\
+0.000 \\
-5.674 \\
+1.900 \\
-0.000 \\
+1.964 \\
-3.017 \\
+2.152 \\
-0.000\end{array}$ & $\begin{array}{r}+3.814 \\
-3.866 \\
+2.889 \\
-2.926 \\
+7.374 \\
-7.450 \\
+16.076 \\
-16.198 \\
+58.361 \\
-58.670\end{array}$ & $\begin{array}{r}+6.149 \\
-0.000 \\
+7.101 \\
-0.000 \\
+6.071 \\
-5.208 \\
+0.096 \\
-14.309 \\
+46.571 \\
-26.008\end{array}$ \\
\hline
\end{tabular}


As presented in Figure 6.1, the dependence of the ratios on both variables $p_{z}$ and $p_{T}$ are visible: the $\pi^{-} / \pi^{+}$and $K^{+} / \pi^{+}$ratios increase with $p_{T}$, whereas the $\pi^{-} / \pi^{+}$, $K^{-} / K^{+}$and $K^{-} / \pi^{-}$ratios decrease with $p_{z}$.

\subsection{Comparison to Other Data and Models}

In this section, the $\pi^{-} / \pi^{+}, K^{-} / K^{+}$and $K^{ \pm} / \pi^{ \pm}$ratios for the NuMI data are compared with the thin Carbon data and the Monte Carlo simulation. In addition, the comparison between the NuMI data and the MINOS models are presented.

Figure 6.2 shows the ratios produced in the NuMI data, the thin Carbon data and the NuMI Monte Carlo simulation as a function of momentum bin. The general dependence in data of the ratios on $p_{z}$ and $p_{T}$ is reproduced in the NuMI Monte Carlo simulation. However, the simulation overestimates the $\pi^{-} / \pi^{+}$and $K^{-} / K^{+}$ ratios, while it underestimates the $K^{+} / \pi^{+}$ratio. The poor agreement between simulation and data indicates that the particle production on the NuMI target is not well simulated.

In comparison to the $\mathrm{p}+\mathrm{C}$ collisions [3], the NuMI data show a steeper structure in $p_{T}$ for $\pi^{-} / \pi^{+}$and $K^{+} / \pi^{+}$, whereas the decreasing slope of $p_{z}$ in $K^{-} / K^{+}$in the NuMI data clearly visible. Also, the NuMI data tend to have higher $K^{+} / \pi^{+}$in most momentum bins than the thin Carbon data. Note however that the thin Carbon data points have asymmetric error bars which are larger in the upper limit. This may indicate the need for a more detailed background study of the thin Carbon data. 


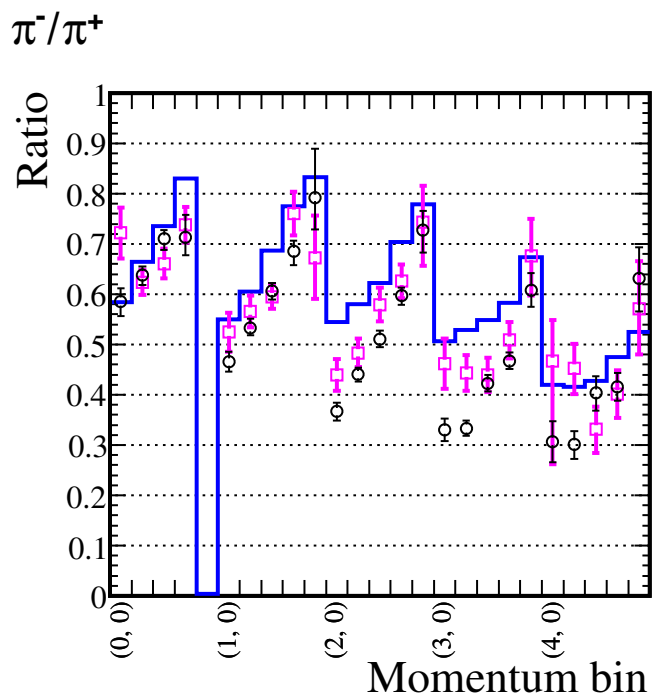

\section{$\mathbf{K}^{-} / \mathbf{K}^{+}$}

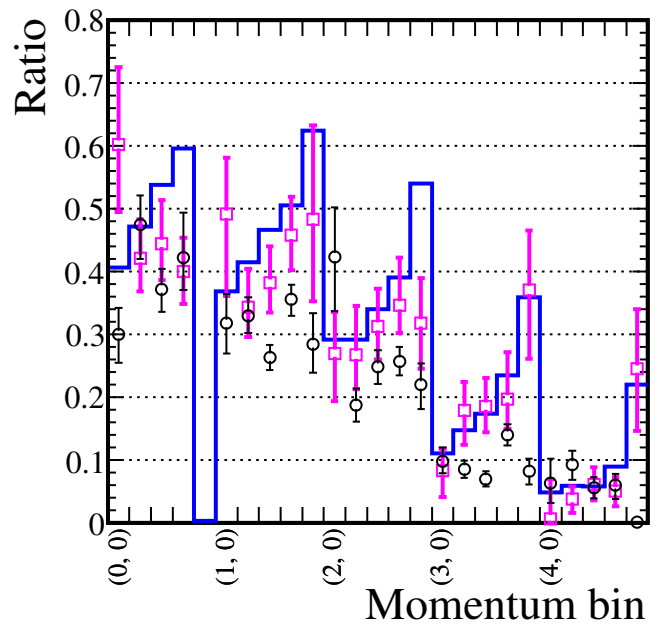

$\mathrm{K}^{+} / \pi^{+}$

$\mathrm{K}^{\top} / \pi^{-}$
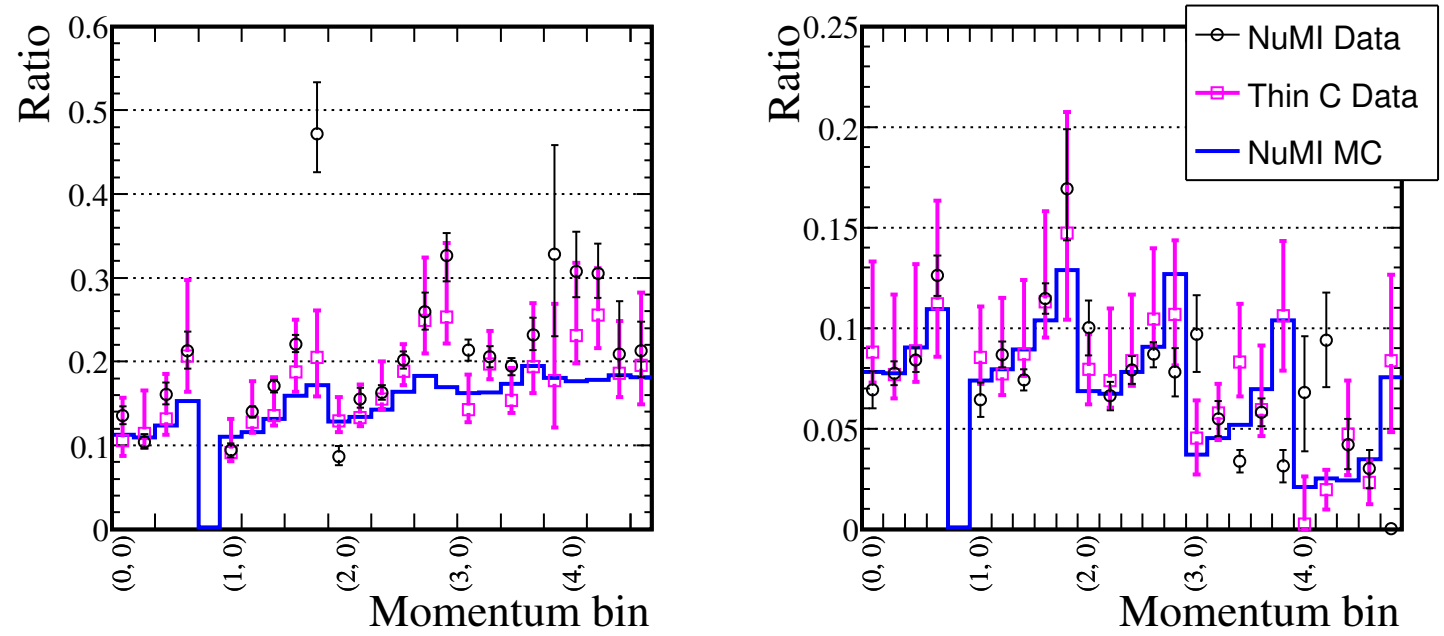

Figure 6.2: The ratios as a function of $\left(p_{z}, p_{T}\right)$ for $\pi^{-} / \pi^{+}, K^{-} / K^{+}$and $K^{ \pm} / \pi^{ \pm}$produced in p+NuMI, p+C collisions and NuMI Monte Carlo simulation at $120 \mathrm{GeV} / c$. 
Figure 6.3 shows the ratios between the NuMI data and the thin Carbon data, and the ratios between the NuMI Monte Carlo simulation and the thin Carbon Monte Carlo simulation. As discussed above, and shown in Figure 6.2, the distributions have steeper slopes in $p_{T}$ in the NuMI data and they are more pronounced than in $\mathrm{p}+\mathrm{C}$ collisions. This feature is discernible in the Monte Carlo ratio of $\pi^{-} / \pi^{+}$in Figure 6.3, although it is difficult to draw any firm conclusion given the errors. It does however demonstrates that the ratios are expected to be smaller than 1 in the Monte Carlo simulation for $\pi^{-} / \pi^{+}$and $K^{-} / K^{+}$, and larger than 1 for $K^{+} / \pi^{+}$, which is consistent with the data measurement in most bins.

Note that the thin Carbon target has an interaction length of $2 \%$, whereas the NuMI target has an interaction length of $90 \%$. The particle ratios from the measurements of proton-Carbon interactions account for only the interactions of the primary protons, and so the effect of re-interactions in the NuMI target should be the primary difference between the two data samples.

The only other relevant models which can be directly compared to the NuMI data are the MINOS models $[4,5]$. In order to assess differences between the MINOS models and the NuMI data, the ratios at different $\left(p_{z}, p_{T}\right)$ presented in Figure 6.1 are replotted for $p_{T}<0.2 \mathrm{GeV} / c$ and $0.2 \mathrm{GeV} / c<p_{T}<0.4 \mathrm{GeV} / c$ as a function of $p_{z}$. This allows a clear view of the substructure at low $p_{T}$. These comparisons are shown in Figure 6.4 and Figure 6.5.

The comparison with the MINOS refit [42], which is driven by MINOS Near de- 


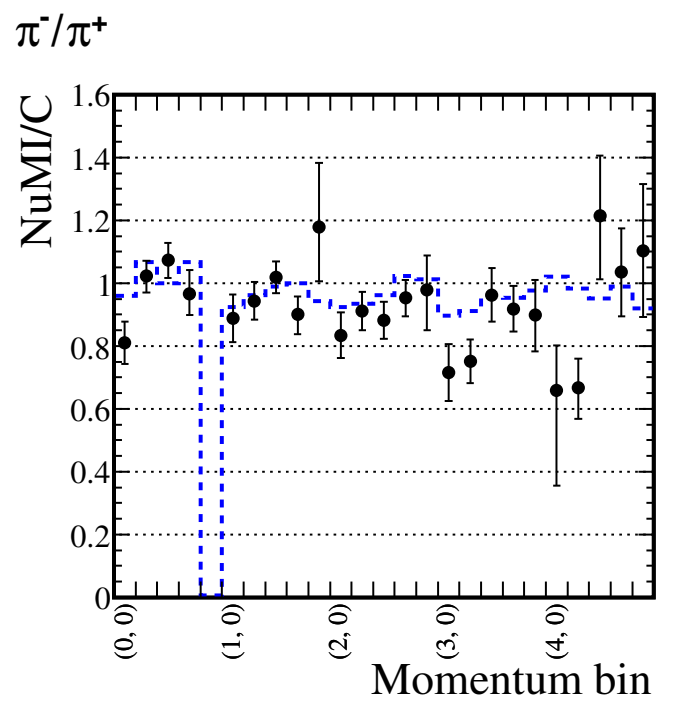

\section{$\mathbf{K}^{-} / \mathbf{K}^{+}$}
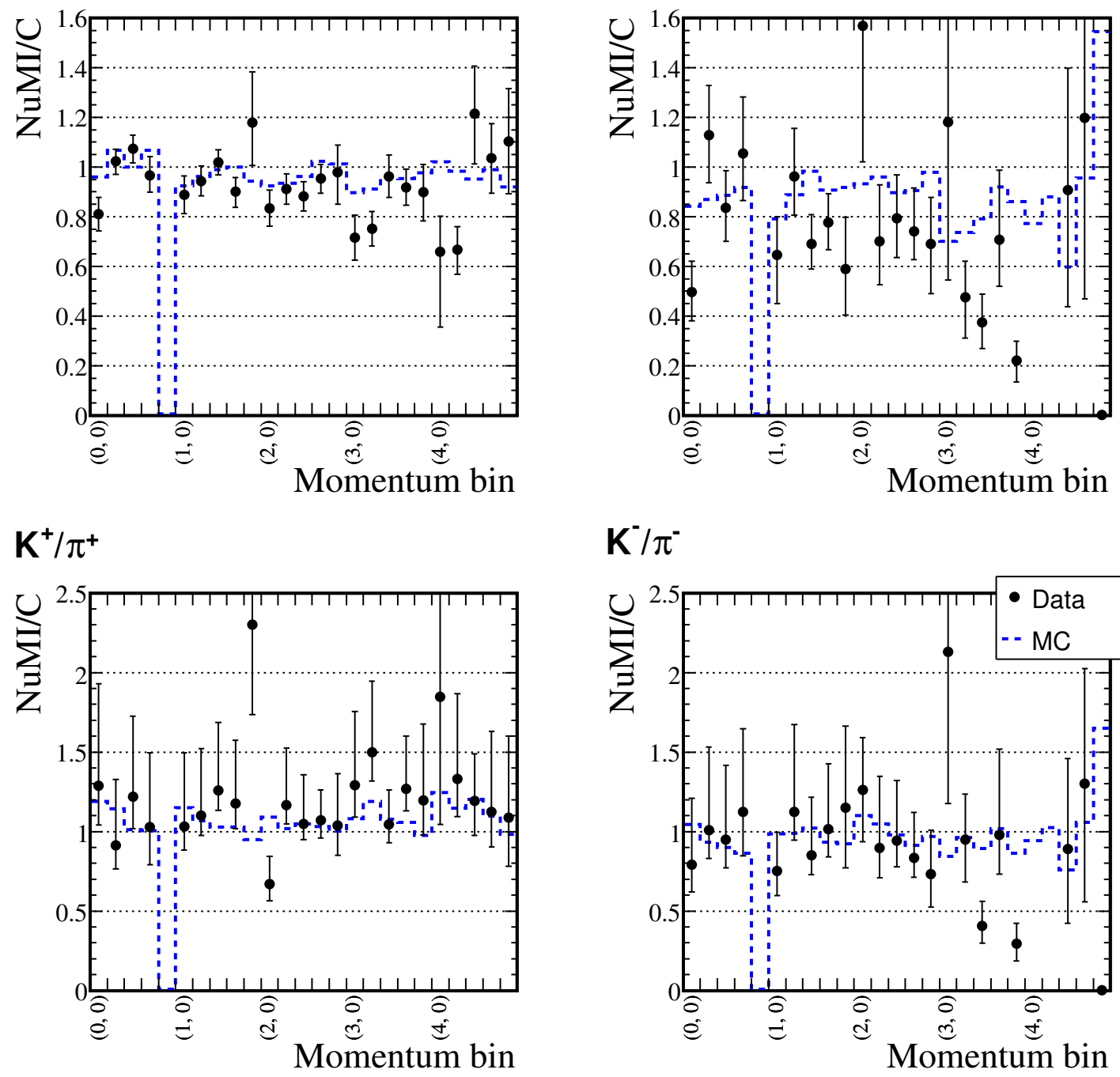

$\mathrm{K}^{-} / \pi^{-}$

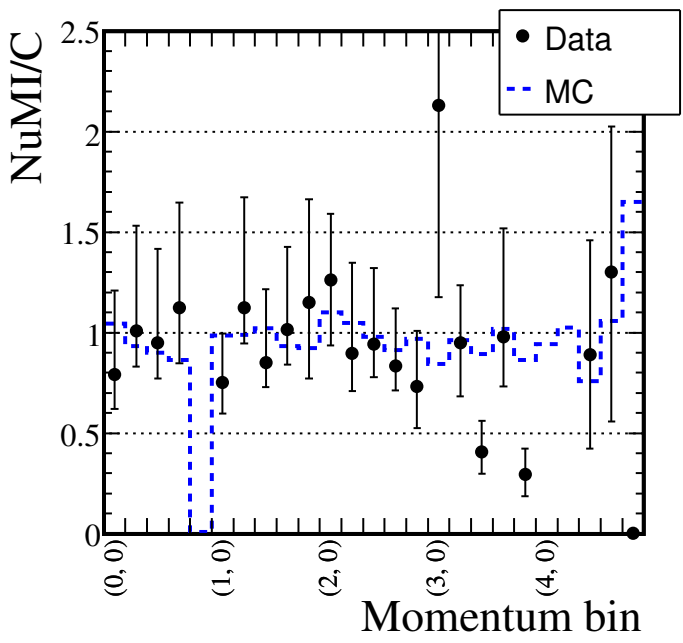

Figure 6.3: The ratios between $\mathrm{p}+\mathrm{NuMI}$ and $\mathrm{p}+\mathrm{C}$ collisions as a function of $\left(p_{z}, p_{T}\right)$ for $\pi^{-} / \pi^{+}, K^{-} / K^{+}$and $K^{ \pm} / \pi^{ \pm}$in data and Monte Carlo simulation at $120 \mathrm{GeV} / c$. Points: data; dashed line: Monte Carlo simulation. 

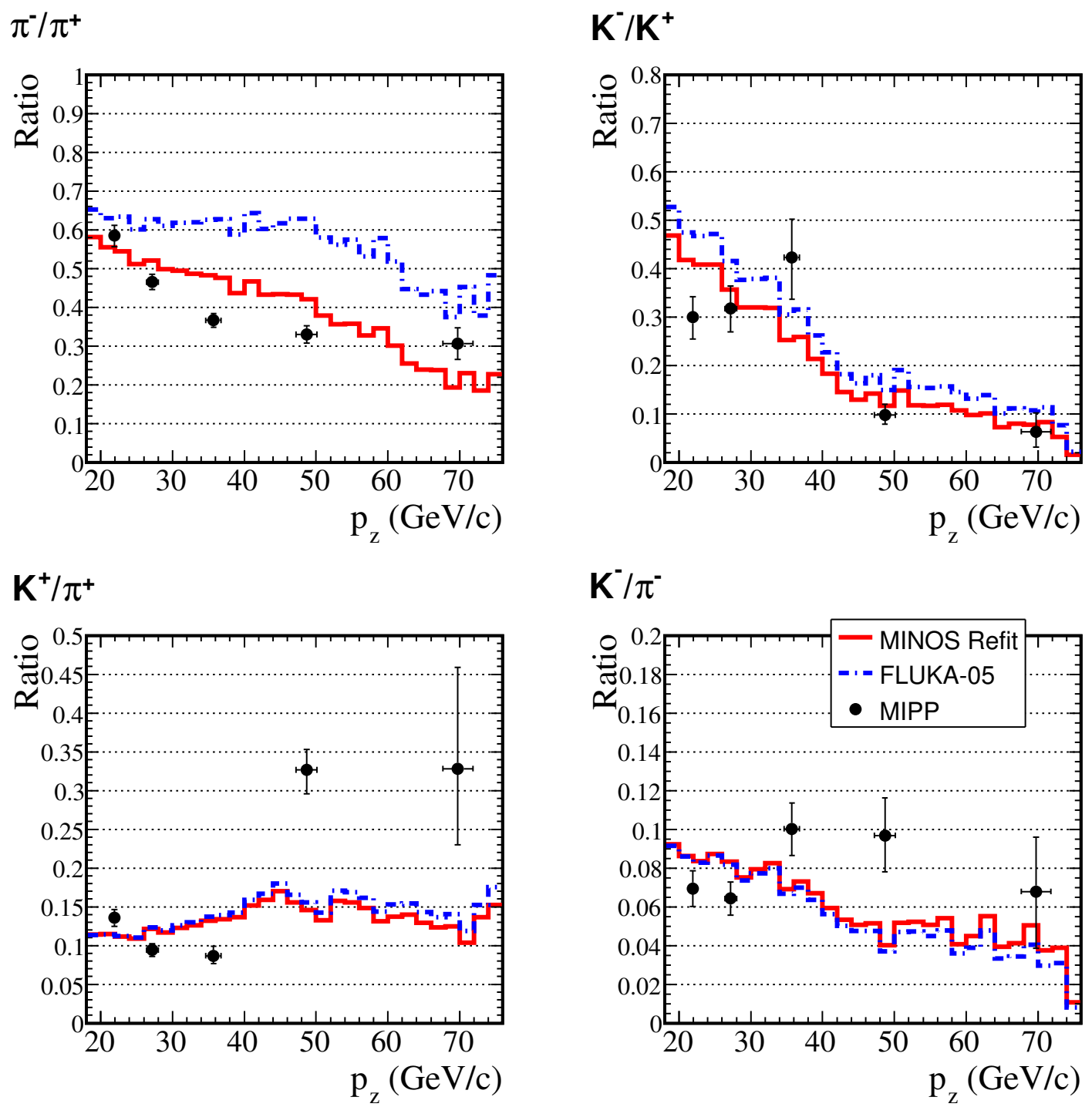

Figure 6.4: The ratios as a function of $p_{z}$ at $p_{T}<0.2 \mathrm{GeV} / c$ for $\pi^{-} / \pi^{+}, K^{-} / K^{+}$and $K^{ \pm} / \pi^{ \pm}$produced in MIPP NuMI data and the MINOS models. 

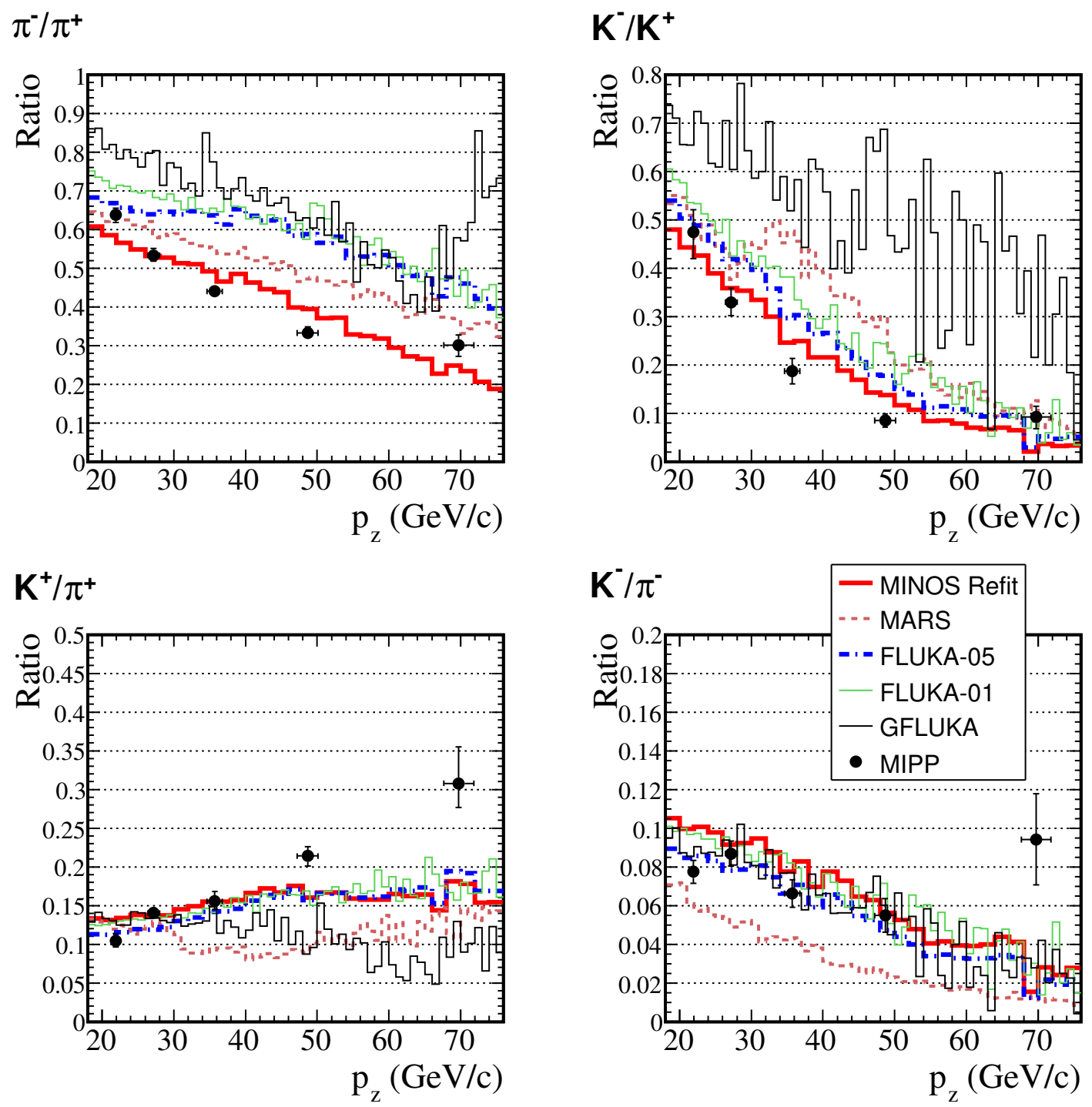

Figure 6.5: The ratios as a function of $p_{z}$ at $0.2 \mathrm{GeV} / c<p_{T}<0.4 \mathrm{GeV} / c$ for $\pi^{-} / \pi^{+}$, $K^{-} / K^{+}$and $K^{ \pm} / \pi^{ \pm}$produced in MIPP NuMI data and the MINOS models. 
tector data, shows reasonable agreement. Remarkable differences are however visible in $K^{ \pm} / \pi^{ \pm}$at high $p_{z}$, where a clear upward deviation with the MINOS refit is evident. It is interesting to note that these data agree with the MINOS refit parametrization significantly better than other models. 


\section{Chapter 7}

\section{Conclusions}

The $\pi^{-} / \pi^{+}, K^{-} / K^{+}, \pi^{+} / K^{+}$and $\pi^{-} / K^{-}$production ratios for proton interactions with the NuMI target observed with the MIPP experiment are presented. The data cover the high momentum region with $p_{T}<2 \mathrm{GeV} / c$ and $20 \mathrm{GeV} / c<$ $p_{z}<90 \mathrm{GeV} / c$. The statistical uncertainties are typically at the $10 \%$ level for $\pi^{-} / \pi^{+}$

and $\pi^{+} / K^{+}$. An iterative, data-driven method is used to evaluate the accuracy of the Monte Carlo simulation and reduce the particle identification background.

These data carry important information concerning understanding of the neutrino spectrum in MINOS from the NuMI beam. The data also provide substantial new input to hadron production models for Monte Carlo simulations. Detailed comparisons with the MIPP thin Carbon data and the Monte Carlo simulations in the corresponding energy range, as well as comparisons to the existing MINOS models, demonstrate that the models in general agree with the data. Some discrepancies exist however, 
particularly for $K^{+} / \pi^{+}$at high $p_{z}$. It is also interesting to note that the MINOS Refit model shows generally better agreement than any other model.

A natural extension to this work would be to compute particle yields from the NuMI target based on the thin Carbon target measurements by taking into account the contribution of cascade processes and tertiary particle production. Also, improvements to the particle identification and background estimation should be possible by using the information from other sub-detectors. This would also improve momentum space coverage. Finally, the MIPP data collected for other thin targets can be used to provide a deeper understanding of hadron production in the Monte Carlo models. 


\section{Appendix A}

\section{Maximization of Purity and}

\section{Efficiency}

In this Appendix, the proof that the likelihood ratio cuts should be tuned to maximize $\mathcal{P} \times \mathcal{E}$ is demonstrated, where $\mathcal{P}=$ Purity and $\mathcal{E}=$ efficiency.

Let us assume the following parameters:

$S_{0}=$ the total number of signal events

$S=$ the measured number of signal events

$B=$ the number of measured background events

$\sigma=$ error on $S$

Using the definitions of purity and efficiency,

$S=\mathcal{E} S_{0}$

$\mathcal{P}=S /(S+B)$. 
Solving for $B$, we have

$B=S(1-\mathcal{P}) / \mathcal{P}$

Given that $\sigma=\sqrt{S+B}$, the fractional error on $S$ is:

$\sigma / S=\sqrt{S+B} / S=\sqrt{S+S(1-\mathcal{P}) / \mathcal{P}} / S=\sqrt{(1+(1-\mathcal{P}) / \mathcal{P}) / S}$

$\sigma / S=1 / \sqrt{\mathcal{P} S}=1 / \sqrt{\mathcal{E P} S_{0}}$.

Therefore, in order to minimize $\sigma / S$, one would need to to maximize $\mathcal{E P}$. 


\section{Bibliography}

[1] The NuMI Facility Technical Design Report. 1998. NuMI-L-337.

[2] MINOS Technical Design Report. 1998. MINOS-doc-337.

[3] Andrey V. Lebedev. Ratio of Pion Kaon Production in Proton Carbon Interactions. PhD thesis, Harvard University, Cambridge, MA, May 2007.

[4] D. G. Michael et al. [MINOS Collaboration]. Observation of Muon Neutrino Disappearance with the MINOS Detectors and the NuMI Neutrino Beam. Phys. Rev. Lett., 97:191801, 2006.

[5] Zarko Pavlovic. private communication.

[6] H. W. Atherton et al. PRECISE MEASUREMENTS OF PARTICLE PRODUCTION BY $400 \mathrm{GeV} / c$ PROTONS ON BERYLLIUM TARGETS. 1980. CERN 80-07.

[7] D. S. Barton et al. Experimental study of the $A$ dependence of inclusive hadron fragmentation. Phys. Rev., D27:2580-2599, 1983.

[8] G. Ambrosini et al. [NA56/SPY]. Measurement of charged particle production from $450 \mathrm{GeV} / c$ protons on beryllium. Eur. Phys. J., C10:605-627, 1999.

[9] M. G. Catanesi et al. Proposal for hadron production measurements using the NA49 detector for use in long-baseline and atmospheric neutrino flux calculations. 2001. CERN-SPSC/2001-017.

[10] Application Software Group. GEANT - Detector Description and Simulation Tool. CERN Program Library Long Writeup W5013, 1994.

[11] A. Fassò, A. Ferrari, J. Ranft, and P.R. Sala. FLUKA: a multi-particle transport code. CERN-2005-10 (2005), INFN/TC_05/11, SLAC-R-773.

[12] A. Fassò, A. Ferrari, S. Roesler, P.R. Sala, G. Battistoni, F. Cerutti, E. Gadioli, M.V. Garzelli, F. Ballarini, A. Ottolenghi, A. Empl and J. Ranft. The physics models of FLUKA: status and recent developments. Computing in High Energy 
and Nuclear Physics 2003 Conference (CHEP2003), La Jolla, CA, USA, March 24-28, 2003, (paper MOMT005), eConf C0303241 (2003), arXiv:hep-ph/0306267.

[13] N. V. Mokhov. The MARS Code System User's Guide. 1995. Fermilab-FN-628.

[14] N. V. Mokhov and O. E. Krivosheev. MARS Code Status. Proc. Monte Carlo 2000 Conf., p. 943, Lisbon, October 23-26 (2000). Fermilab-Conf-00/181.

[15] N. V. Mokhov. Status of MARS Code. 2003. Fermilab-Conf-03/053.

[16] N. V. Mokhov, K. K. Gudima, C. C. James et al. Recent Enhancements to the MARS15 Code. 2004. Fermilab-Conf-04/053; http://www .ap.fnal .gov/MARS/.

[17] M. Bonesini, A. Marchionni, F. Pietropaolo and T. Tabarelli de Fatis. On particle production for high energy neutrino beams. Eur. Phys. J., C20:13-27, 2001.

[18] A. J. Malensek. Empirical Formula for Thick Target Particle Production. 1981. Fermilab-FN 341.

[19] A. Abramov et al. Dynamic Stress Calculations for ME and LE Targets and Results of Prototyping for the LE Target. 2000. MINOS-doc-675.

[20] Xihong Yang. Centrality dependence of strangeness production in proton-nucleus collisions at AGS energies. PhD thesis, Columbia University, 2000.

[21] K. Kodama et al. Identification of neutrino interactions using the donut spectrometer. Nucl. Instrum. Meth., A516:21-33, 2004.

[22] M. De Palma et al. A SYSTEM OF LARGE MULTIWIRE PROPORTIONAL CHAMBERS FOR A HIGH INTENSITY EXPERIMENT. Nucl. Instrum. Meth., 217:135-139, 1983.

[23] D. C. Christian et al. High rate drift chambers. Nucl. Instrum. Meth., A345:62$71,1994$.

[24] J. Engelfried et al. [E781]. The E781 (SELEX) RICH detector. Nucl. Instrum. Meth., A409:439-442, 1998.

[25] J. Engelfried et al. [SELEX]. The SELEX phototube RICH detector. Nucl. Instrum. Meth., A431:53-69, 1999.

[26] J. Engelfried et al. The RICH detector of the SELEX experiment. Nucl. Instrum. Meth., A433:149-152, 1999.

[27] G. Rai et al. A TPC detector for the study of high multiplicity heavy ion collisions. IEEE Trans. Nucl. Sci., 37:56-64, 1990. 
[28] Stuart A. Kleinfelder. A 4096 cell switched capacitor analog waveform storage integrated circuit. IEEE Trans. Nucl. Sci., 37:1230-1236, 1990.

[29] K. L. Lee et al. Analog-to-Digital Conversion Using Custom CMOS Analog Memory for the EOS Time Projection Chamber. IEEE Trans. Nucl. Sci., 38:344347, 1991.

[30] Fred Bieser, Ron Jones and Charles McParland. Data Links for the EOS TPC. IEEE Trans. Nucl. Sci., 38:335-336, 1991.

[31] M. Nakamura, D. A. Landis and G. Rai. Low Power Shaper Amplifier for the EOS TPC Detector. IEEE Trans. Nucl. Sci., 38:50-52, 1991.

[32] H. Wieman et al. A TPC detector for the study of high multiplicity heavy ion collisions. Nucl. Phys., A525:617c-620c, 1991.

[33] H. Wieman et al [EOS]. Heavy Ion Reaction Measurements with the EOS TPC (Looking for Central Collisions with Missing Energy). Presented at Winter Workshop on Nuclear Dynamics, Snowbird, UT, Jan 15-21, 1994.

[34] R. A. Burnstein et al [HyperCP]. HyperCP: A high-rate spectrometer for the study of charged hyperon and kaon decays. Nucl. Instrum. Meth., A541:516-565, 2005.

[35] J. B. Lindsay, C. Millerin, J. C. Tarle et al. A FAST AND FLEXIBLE DATA ACQUISITION SYSTEM FOR MULTIWIRE PROPORTIONAL CHAMBERS AND OTHER DETECTORS. Nucl. Instrum. Meth., 156:329-333, 1978.

[36] J. Paley, R. Soltz, P. D. Barnes, M. Heffner, J. Klay and D. Miller. MIPP TPC Reconstruction. 2006. MIPP-doc-129.

[37] R. Fernow. Introduction to experimental particle physics. Cambridge University Press, 1986.

[38] A. Bideau-Mehu, Y. Guern, R. Abjean and A. Johannin-Gilles. Interferometric determination of the refractive index of carbon dioxide in the ultraviolet region. Optics Communications, 9(4):432-434, 1973.

[39] The SELEX collaboration. private communication.

[40] Peter L. Smith and Kouichi Yoshino. private communication.

[41] M. Starič and P. Križan. An iterative method for the analysis of Cherenkov rings in the HERA-B RICH. Nucl. Instrum. Meth., A433:279-285, 1999.

[42] S. Kopp, R. Ospanov, Z. Pavlovich and T. Vahle. Constraining the beam neutrino mc flux using the minos nd data. 2007. MINOS-doc-2965. 